\title{
Whole-genome epigenetic function annotation through sgRNA libraries synthesized by controlled template-dependent elongation
}

Junling Jia ( $\sim$ junling.jia@alumni.bcm.edu )

The First Affiliated Hospital, Zhejiang University

\section{Chen Pan}

ZheJiang Univeristy

\section{Ran Li}

life sciences institute zhejiang university

\section{Liyan Shui}

Collaborative Innovation Center for Diagnosis and Treatment of Infectious Diseases, State Key

Laboratory for Diagnosis and Treatment of Infectious Diseases, The First Affiliated Hospital

\section{Yali Wang}

life sciences institute zhejiang university

\section{Zhengyun Xiao}

zhejiang university

Jing Zhu

Mingtian Genetics

\section{Chao Wu}

Zhe Jiang Univerisity https://orcid.org/0000-0002-6193-4398

\section{Min Zheng}

ZheJiang Univeristy

\section{Article}

Keywords: Genetic screen, CRISPR/Cas9, sgRNA library, H3K4me3, CTCF, mESCs, Liver cancer, LincRNA, CDC42

Posted Date: November 5th, 2020

DOI: https://doi.org/10.21203/rs.3.rs-100657/v1

License: (c) (i) This work is licensed under a Creative Commons Attribution 4.0 International License.

Read Full License 


\section{Whole-genome epigenetic function annotation through} 2 sgRNA libraries synthesized by controlled template-

\section{3 dependent elongation}

4

5

6

7

8

9 Liver cancer, LincRNA, CDC42 Beijing, 100166, PRC Junling.jia@alumni.bcm.edu (J.J.)

\# These authors contributed equally

$$
\text { Chao } \mathrm{Wu}^{2, *} \text {, Min Zheng }{ }^{2, *} \text { and Junling } \mathrm{Jia}^{2,4,{ }^{*}}
$$

1. Life Sciences Institute, Zhejiang University, Hangzhou, Zhejiang, 310058, PRC

2. Collaborative Innovation Center for Diagnosis and Treatment of Infectious Diseases, State Key Laboratory for Diagnosis and Treatment of Infectious Diseases, The First Affiliated Hospital, Zhejiang University, Hangzhou, Zhejiang 310003, PRC Zhejiang University, Hangzhou, Zhejiang 310003, PRC

3. Department of Anesthesiology \& Center for Shock, Trauma and Anesthesiology Research, University of Maryland School of Medicine, Baltimore, MD, 21201, USA

4. Innovation Center for Precision Medicine, Zhongtong-Lanbo Diagnostic LTD,

*. Correspondence: minzheng@zju.edu.cn (M.Z.), wuchao1984@zju.edu.cn (C.W.),

Keywords: Genetic screen, CRISPR/Cas9, sgRNA library, H3K4me3, CTCF, mESCs, 3 5 
Pan et al 2020

26 Abstract

27

28 Epigenome is the set of DNA-associated proteins or chemical modifications to DNA,

29 which regulates gene expression in processes of development and disease. While

30 current advances have allowed researchers to routinely profile epigenomes from given

31 samples, our understandings of the functions of epigenetic hallmarks are nonspecific at

32 best. Applying CRISPR-screening to genome-widely interrogate the function of

33 individual epigenetic hallmarks demands massive sgRNA libraries which are

34 unaffordable via commercial syntheses. Our development consists of a high throughput

35 and cost-effective controlled template-dependent elongation (CTDE) approach which

36 converts source DNA to sgRNA templates. Affiliated screenings encompass $3.8 \mathrm{M}$

37 sgRNAs generated by CTDE targeting all major H3K4me3 and CTCF hallmarks in

mESCs and HepG2 and identified 20K essential epigenetic hallmarks, which render the

first batch of functional epigenome annotation of $\mathrm{H} 3 \mathrm{~K} 4 \mathrm{me} 3$ and CTCF hallmarks in

40

mammals. As an application example, we show that a H3K4me3 hallmark orchestrates

41 CDC42 level and cell-cycle progression through promoting LINC00339 expression in

42 HepG2.

43 
Pan et al 2020

\section{Introduction}

Humans have over 20,000 protein-coding genes, which account for about $2 \%$ of the overall genome $\mathrm{DNA}^{1}$. The expression of those genes are modulated by elements in the remaining portions of the genome, which are regarded as the regulome - an assortment of chromatin components, such as promoters, transcriptional regulatory regions, and high dimensional chromatin structures ${ }^{1,2}$. Being a major component of regulome, epigenome contains the set of DNA-associated proteins or chemical modifications to $\mathrm{DNA}^{3}$. Systematic discovery and location annotation of epigenome are accomplished primarily by Consortiums such as ENCODE and Roadmap Epigenomics

Mapping through assays such as DNase hypersensitivity assays, DNA methylation assays and chromatin immunoprecipitation sequencing (ChIP-seq) assays ${ }^{4-8}$. These refined techniques have allowed most labs to routinely profile their interested epigenomic information under deliberate experimental conditions.

The function of many epigenetic modifications is generally known. For example, H3K4me3 (tri-methylation at the 4th lysine residue of the DNA packaging protein Histone H3) is involved in the positive regulation of the nearby gene transcription ${ }^{9,10}$. H3K4me3 plays a significant role regarding the regulation of stem cell lineage potency ${ }^{11}$ as well. CTCF hallmarks primarily have a CCCTC-like motif and are bound by CTCF protein, a 11-zinc finger protein ${ }^{12}$. Moreover, CTCF hallmarks are involved in various cellular processes such as transcriptional regulation, insulator activity, V(D)J recombination and the regulation of chromatin architecture ${ }^{13-17}$. 

However, there is still a scarcity of a functional epigenome annotation of these hallmarks considering genetic screens have prioritized protein-coding genes or expressed non-coding loci ${ }^{18,19}$. function of regulatory elements using designed dense tiling sgRNA libraries targeting

73 limited genomic loci ${ }^{8,20,21}$. While these studies have provided proof of concept for the application of pooled CRISPR-screening in the functional characterization of

75 regulatory elements, the high-cost of synthesizing a dense tiling sgRNA library to cover an epigenetic hallmark genome-widely (around half-million USD for H3K4me3 or

77 CTCF) is unrealistic and a major hurdle to further functional epigenome studies. elongation (CTDE) approach that can convert any DNA sample to a sgRNAs library, which covers $98.47 \%$ of the effective CRISPR/Cas9 targeting sites within the source DNA. Significantly, over 99\% CTDE-sgRNAs targeting sequences have a protospacer adjacent motif (PAM) $)^{22-24}$. We have generated sgRNA libraries targeting all H3K4me3 and CTCF hallmarks in mESCs and HepG2. In total, we have screened 3.8 M sgRNAs and identified 14K (14265) H3K4me3 and 6K (6235) CTCF essential hallmarks for the

85 proliferation of mESCs and HepG2. mESCs CTCF dataset shows that mESCs 
maintains a high proportion of non-essential cell-type specific CTCF hallmarks, which

87 may be important for the implement of pluripotency. Importantly, the HepG2

$88 \mathrm{H} 3 \mathrm{~K} 4 \mathrm{me} 3$ dataset helps confirm that an essential H3K4me3 hallmark inside the intron

89 of LINC00339 orchestrates the cell-cycle progression and the expression of CDC42, a

90 pivotal factor for the proliferation and invasion of cancer cells, through promoting

91 LINC00339 expression.

92 Our studies have brought us to develop an efficient and budget-friendly approach

93 (CTDE) to convert DNA to a sgRNA library. Several breakthroughs have ensued, one

94 of them being the first batch of functional epigenome annotation of H3K4me3 and

95 CTCF hallmarks in mammals through CTDE library coupled CRISPR-screening.

96 Various other findings through characterizing an essential H3K4me3 hallmark in

97 HepG2 have shown the significance of functional epigenome annotation in cancer 98 research.

99

100

101

102 
103

104

105

106

107

108

109

110

111

113

115

116

117

118

119

120

121 incorporation before the restoration of their $3^{\prime}$-hydroxy groups (Figure 1a) ${ }^{28}$. We

122 restored the 3 'hydroxyl group via tris (2-carboxyethyl) phosphine (TCEP) treatment

123 and repeated another round of nucleotide incorporation following the previous

124 extension (Figure 1a) ${ }^{28}$. After 23 rounds of cycling, we blunt the $3^{\prime}$ terminus to get $23 \mathrm{bp}$ 125 DNA fragments (not including the adapter) (Figure 1a). The most used Cas9 from

Convert DNA to a sgRNA library through synthesized by controlled templatedependent elongation (CTDE)

The CRISPR/Cas9 system has been developed into genome mutating tools with wide-ranging applications ${ }^{25}$. The single guide RNA (sgRNA) binds to the Cas9 enzyme and guides the complex to a target via pairing the complementary DNA sequences followed by a protospacer adjacent motif (PAM), where Cas9 performs its endonuclease activity immediately ${ }^{22,25}$. Currently, sgRNA templates are designed as 17-22bp DNA sequences and commercially synthesized in vitro as ready-to-clone fragments ${ }^{25-27}$.

To directly generate large-scale sgRNA templates from source DNA, we fragmented the DNA template within a $1 \mathrm{~kb}$ length (Figure 1a). We then ligated the DNA fragments with A1 adaptors (red), immobilizing them on streptavidin beads and washing away their positive strands under a denaturing condition (Figure 1a). Next, we annealed the priming primer (the positive strand of A1 adaptor) onto the immobilized minus strand, then extending the primer using DNA polymerase coupled with reversible terminator (RT) nucleotides (3'-O-N $3-d N T P)$ that allow singular nucleotide (x)ension (Figure 1 a) 28. After 23 rounds of cycling, we blunt the $3^{\prime}$ terninus to get 23 bp 
Streptococcus pyogenes recognizes the 5'-NGG-3" (where "N" can be any nucleotide

127 base) PAM sequence. If the last two nucleotides of 3'end of the 23bp DNA are GG, it

128 will compose an AscI cutting site after the A2 adaptor ligation, which is used to select

129 the DNA fragment with 5'-NGG-3' PAM (Figure 1a). Following PAM selection, we

130 remove the NGG triplet using a type II restrict endonuclease (BbsI) and put an A4

131 adaptor onto the $3^{\prime}$ terminus for further Gibson assembly into a sgRNA expressing

132 vector (Figure 1a) ${ }^{29}$. Since only two rare-cutting endonucleases are employed, the

133 dropout rate of sgRNA template caused by endonuclease cutting is very low $(0.86 \%$

134 per mouse genome and $0.82 \%$ per human genome).

135

To test the efficiency of this technique, we used a $14.9 \mathrm{~kb}$ plasmid (lentiCRISPR-

136 v2) modeling a DNA template. After implementing the CTDE steps described above

137 (Figure 1a and S1a), we generated sgRNAs targeting $98.47 \%$ of the sites with 5'-NGG-

138 3' PAM sequence (Figure $1 \mathrm{~b}$ and $\mathrm{S} 1 \mathrm{~b}$ ). As expected, few sgRNAs can be generated

139 from AT rich region because of the low complimentary binding affinity between AT

140 rich sequences (Figure S1b). We also checked the capability of CTDE to enrichment

141 the DNA fragments with 3' NGG triplet. There are $6.23 \%$ input DNA fragments that

142 are adjacent to a $3^{\prime}$ NGG triplet, and after enrichment, the rate is raised to $99.7 \%(15.98$

143 folds) (Figure 1c). As expected, the lengths of the sgRNA templates are predominantly

$14420 \mathrm{bp}(83.93 \%)$ and the functional sgRNA templates (17-22bp) are 98.66\% (Figure $1451 \mathrm{~d})^{26,27}$. 

compared with the popular sgRNA libraries (GeCKO 2.0 human/mouse) generated by perfectly match a position in their template, and 1.6\% sgRNAs of CTDE library carry one mismatch (Figure 1e). The error rate of the CTDE procedure is around 0.97 bases per 1000 bases. Around $90 \%$ sgRNAs of CBDS can perfectly match their targeting positions and around 7.5\%/9.5\% sgRNAs carry one mismatch (Figure 1e). The CBDS

153 library, having an error rate of around 4.5-5.7 bases per 1000 bases, assimilates itself

154 with the CTDE library. We also compared the amplification bias between CTDE library 155 and CBDS library. The sgRNA abundance of CBDS library and CTDE library are both 156 similarly low (Figure 1f). Treating of the abovesaid data, CTDE, consistent in the 157 conversion of source DNA to sgRNA library, produces the same qualitative results as 158 the library generated via Chip Based DNA Synthesis (CBDS).

159 Validations of CTDE sgRNA library screening

160 To implement mega-level screenings, we planned to infect cells with the lenti-viral

161 CTDE library expressing sgRNAs along with Cas9 protein (around 40K sgRNAs per 162 batch) (Figure 2a). Then, we sequence the abundance of each sgRNA template at two 163 time points (3 days selected in puromycin media as P1 and 20 days expanded after 164 puromycin selection as P10) to calculate the abundance change from P1 to P10 of each 165 sgRNA template (Figure 2a; details in method). 
167 devoted to identifying genes rather than non-coding genomic loci ${ }^{30}$. A coding gene is targeted usually by multiple sgRNAs with similar mutational abilities in traditional analysis abundance change of these sgRNAs in frequented algorithms, such as

$171 \mathrm{MAGeCK}^{31}$. For the purpose, however, of screening non-coding regulatory loci inside epigenetic hallmarks using CTDE library, the objective becomes the identification of narrow essential genomic sites, the majority of said can only be efficiently targeted by one sgRNA. Traditional calling algorithms are unreliable in this scenario as it will report numerous false positive significantly changed sgRNA (ssgRNA) (Figure 2b). During the screening, many minor uncertain factors bias the abundance of each sgRNA randomly and cause the abundance change of the sgRNAs following a normal

178 distribution in the scenario of non-selection pressure ${ }^{32}$. Some sgRNA disruptions will 179 cause a negative selection pressure, bias their abundance in P10 systematically $(<20 \%)$, 180 and result in their abundance change following another normal distribution. While the 181 distance of above two normal distribution is large enough (Figure S2a), we can 182 efficiently identify the $\operatorname{sgRNAs}(\mathrm{FDR}<0.1)$ that leads to negative selections using a self183 developed straight-forward approach (NSgRNAShot; details in method), which has $18496.42 \%$ precision rate and $96.27 \%$ recall rate on a simulation dataset (Figure S2a; Table 185 S1). Most importantly, on identical testing datasets, NSgRNAShot has significantly lower false positive rates than MAGeCK (Figure 2b). Additionally, we compared the 
187

188 189 190 191 192

ability of NSgRNAShot to call true positive ssgRNA with MAGeCK using two published essential gene screen datasets. Due to significant false positive rates, MAGeCK reports a noticeably higher amount of ssgRNAs than NSgRNAShot, most of which target non-essential genes (Figure 2b-c), whereas major portions of ssgRNA identified by NSgRNAShot target essential genes and overlap (100\%) with ssgRNAs targeting essential genes reported by MAGeCK (Figure 2c).

Following this, through targeting a drug resistance gene (Neo) in mESCs (Figure S2b), we compared the efficiency of CTDE library with the library designed via standard CBDS methods (details in method). The CBDS library has 115 sgRNAs targeting all possible sites (NGG PAM) in Neo gene, along with 20 non-targeting control gRNAs. The CDTE library was generated from the abovesaid Neo gene fragments with additions of the same 20 non-targeting control gRNAs (Figure S2b). The mESCs (expressing Neo) were infected with above lenti-viral libraries and were cultured in medium with Neomycin, and the screening was performed according to the abovesaid procedure. As expected, the abundance of Neo targeting sgRNAs of both libraries exhibit significant decrease and can be identified as ssgRNAs (Figure 2d). Most importantly, the ssgRNAs from both libraries are highly overlapped (97\%) (Figure 2d), exhibiting implications that sgRNA library generated by CTDE performs similarly, if not better as the library produced by standard method such as CBDS. Taken together, multiple validations have shown that our CTDE sgRNA library screening approach is both practicable and efficient. 


\section{Annotation of the essential H3K4me3 hallmarks for mESCs self-renewal} under a biological context $\mathrm{t}^{21,33}$.

To genome-widely interrogate essential regulatory elements within H3K4me3 hallmarks during mESCs self-renewal, we first acquired the H3K4me3 labeled DNA fragments through chromatin immunoprecipitation (ChIP) and converted them to sgRNA libraries through CTDE (Figure 1a). To implement the screening, we infected the mESCs with the lenti-viral library expressing sgRNAs along with Cas9 protein

221 (around 40K sgRNAs per batch). Then, we performed the screening as described above 222 (Figure 2a).

In total, we screened $926 \mathrm{~K}$ sgRNAs targeting $82.99 \%$ of the H3K4me3 enriched

224 regions (Figure 3a-d; Table S2-3). In H3K4me3 highly enriched regions (top 100), the 225 sgRNA density is high and reaches to 59 sgRNA per $\mathrm{kb}$. The abundance distribution of 226 sgRNAs inside H3K4me3 hallmarks observes the pattern of their template DNAs

227 (Figure 3a-b and S3a). We identified 24189 sgRNAs causing significant negative 228 selection (called ssgRNA from here), which indicates that their targeting sites in 
229 230 231 232

H3K4me3 hallmarks are essential regulatory elements for mESCs self-renewal (Figure 3a-d; Table S4). We verified three randomly picked ssgRNAs in non-coding regions. As expected, all three ssgRNAs can significantly inhibit the proliferation of mESCs (Figure 3e and S3b-c).

H3K4me3 ssgRNAs distribute evenly on most chromosomes, regions on chromosome 6, 9 and $\mathrm{X}$ being an exception (Figure 3a). Given these biases are not the results of lacking matrix $\mathrm{H} 3 \mathrm{~K} 4 \mathrm{me} 3$ hallmarks or sgRNAs (Figure 3a), we reason that these regions contain fewer essential genes regulated by H3K4me3 hallmarks. ssgRNAs appear in regions, arrayed from weak to strong H3K4me3 elements, the majority of which are among strong elements regions (Figure 3b). Among all essential H3K4me3 elements, $63.24 \%$ of the elements are targeted by 1 ssgRNA, and the remaining $37.76 \%$ are targeted by multiple ssgRNAs (Table S3). Detailed positions of ssgRNAs inside their H3K4me3 elements can be found in the Supplemental table 4. Given that the H3K4me3 elements are generally wide, the location of these ssgRNAs should reflect the core regulatory sites within the elements.

Many H3K4me3 hallmarks locate on exons (Figure 3c; Table S4). Exon mutations will inactivate their genes and cause stronger phenotypes rather than disrupting H3K4me3's regulatory elements. Consistent with this supposition, the percentage of ssgRNAs on exons is significantly greater than the percentage of their matrix sgRNAs, whereas the percentage of the ssgRNAs on other regions are similar as the percentage of their matrix sgRNAs (Figure 3c). Because the sgRNAs targeting known essential 
Pan et al 2020

250 genes should be efficiently identified as ssgRNAs, the exons targeting sgRNAs can be

251 used as spike-ins, providing further validation to the CTDE approach under an authentic

252 mega-library screening condition. In sum, exons of 8165 genes are targeted, while 659

253 known essential genes are included (Figure 3f). As expected, our studies exhibit the

254 ssgRNAs targeting 534 essential genes from the said 659, strongly accrediting the

255 success of our screening (Figure 3f).

256 The enriched Gene Ontology (GO) terms of ssgRNAs targeting exons are related

257 to essential biological processes such as ncRNA metabolic and ribonucleoprotein

258 biogenesis (Figure S3d; Table S5). The corresponding genes of ssgRNAs targeting

259 proximal promoters and UTRs can also be confidently identified (Details in method),

260 and their enriched GO terms are related to the essential biological processes of cell

261 survival as well (Figure S3e-f; Table S5).

262 Thus, we have successfully performed a genome-wide CRISPR-screening to

263 interrogate the essential $\mathrm{H} 3 \mathrm{~K} 4 \mathrm{me} 3$ regulatory elements for mESCs self-renewal.

264

265 Annotation of the essential CTCF hallmarks for mESCs self-renewal

266 Gene expressions are orchestrated by regulatory elements at local, long-range

267 and high-dimensional levels ${ }^{34-36}$. CTCF stabilizes chromosomal architecture and

268 coordinates the genome spatial positioning, which functions as a transcriptional

269 activator or repressor ${ }^{16}$. In addition to local level regulatory elements (H3K4me3), 
interrogating CTCF hallmarks provide an important understanding for gene regulation

271 mechanisms in a biological context.

272 Mouse epigenome has around 55,000-65,000 CTCF hallmarks ${ }^{37}$. $\sim 50 \%$ of them

273 are intergenic and $\sim 35 \%$ of them are intragenic ${ }^{37}$. To genome-widely interrogate

274 essential CTCF hallmarks during mESCs self-renewal, we converted CTCF ChIPed

275 DNA fragments to sgRNA libraries via CTDE (Figure 1a) and performed the CRISPR-

276 screening as described above (Figure 2a). In total, we screened 848K sgRNAs which

277 targets $64.12 \%$ of the CTCF hallmarks in mESCs (Figure 4a-c and S4a-b; Table S3 and

278 S6). The sgRNA density is 24 sgRNAs per kb in CTCF strong binding sites (top 100).

279 As CTCF elements display a consistent size, whilst maintaining a moderate diversity

280 of the input DNA amount, that of which is significantly smaller than the input amount

281 of H3K4me3, the abundance distribution of sgRNAs inside CTCF hallmarks is notably

282 more even than that of $\mathrm{H} 3 \mathrm{~K} 4 \mathrm{me} 3$, although the pattern of their template DNAs is still

283 observed (Figure 4b and S4a). We identified 3038 CTCF ssgRNAs, which indicates

284 that the corresponding CTCF hallmarks (47.02\% in intergenic regions) are essential

285 for mESCs self-renewal (Figure 4a-c and S4b; Table S4). ssgRNAs appear in regions,

286 arrayed from weak to strong CTCF elements. Among all essential CTCF elements,

$28787.07 \%$ elements are targeted by 1 ssgRNA whilst $12.93 \%$ elements are targeted by

288 multiple ssgRNAs (Table S3). Detailed positions of ssgRNAs inside CTCF elements

289 can be found in the Supplemental table 4. CTCF elements are narrow $(83 \%<400 \mathrm{bp})$

290 and have clear binding motif, and the ssgRNAs are rather important in indication of the 
291

292

293

294

295

296

297

298

299

300

301

302

303

304

305

306

307

308

309

310

essentiality of their belonging CTCF elements than their targeting location inside. We verified three randomly picked intergenic CTCF ssgRNAs. As expected, all can significantly compromise mESCs proliferation (Figure $4 \mathrm{~d}$ and $\mathrm{S} 4 \mathrm{c}-\mathrm{d}$ ).

Because CTCF hallmarks stabilize high-dimensional architecture of chromosome at multiple levels, disrupting CTCF hallmarks will disrupt the topological structure of genomes at different levels and can cause different level cell stresses. Thus, the distribution of CTCF ssgRNA on most chromosomes is not even as the distribution of their matrix sgRNAs (Figure 4a-b).

Distal promoters, introns and proximal promoters are major parts on which CTCF hallmarks locate (Figure S4b; Table S4). The GO terms of genes close to ssgRNAs targeting introns and proximal promoters are essential biological processes and tissue developments (Figure 4e and S4e; Table S7). Because the expression of differentiation and development related genes generally antagonizes mESCs pluripotency and selfrenewal, we believe that these essential CTCF hallmarks should inhibit their expression. Unlike H3K4me3 ssgRNAs, only a minor part of CTCF ssgRNAs target exons (Figure S4b; Table S4). Although most GO terms of expressed genes of these exons are also related to essential biological processes, their significance is much lower than that of H3K4me3 ssgRNA (Figure S4e and S3d; Table S5 and S7). We reason that major functions of these essential CTCF hallmarks are beyond promoting the expression of their sitting genes. 
Pan et al 2020

mESCs differentiate into various cell types during embryonic development.

312 Previous studies have shown that the chromosome spatial structure will rearrange

313 accordingly to fit the change of gene expression patterns during differentiation ${ }^{38}$. We

314 compared the CTCF hallmarks with 16 mouse cell types/tissues and found that $59.63 \%$

315 CTCF hallmarks in mESCs are cell-type specific and $40.37 \%$ are common (Figure 4f;

316 Table S8) ${ }^{37,39}$. The common CTCF hallmarks should help maintain the universal spatial

317 structure of chromosome, while the cell-type specific CTCF hallmarks should be either

318 mESCs specific or pre-loaded hallmarks for further differentiated cells. Consistent with

319 this supposition, the percentage of the cell-type specific essential CTCF hallmarks

$320(28.85 \%)$ of mESCs is significantly smaller than the percentage of the cell-type specific

321 CTCF hallmarks (59.63\%) (Figure 4f; Table S8).

322

323 Annotation of the essential H3K4me3 hallmarks in human liver cancer cells

324 Whole-genome sequencing has surveyed large sets of cancer genomes and studied

325 the role and extent of single-nucleotide variants (SNVs), small insertions/deletions

326 (indels) and larger structural variants in cancers ${ }^{40,41}$. While the initial focus on the

327 genetic variations in protein-coding regions has dramatically expanded our knowledge

328 of cancer genetics, the remaining (>90\%) non-coding part of the genetic variations are

329 much more difficult to understand and have remained largely unexplored ${ }^{42}$, which is

330 due to a lack of functional annotation of regulatory elements inside. 
Pan et al 2020

liver cancer cells (HepG2) (Figure S5a-b), we performed a H3K4me3 CRISPR-

333 screening as described above (Figure 1a and 2a). In total, we screened 1.19M sgRNAs

334 targeting $80.91 \%$ of the H3K4me3 hallmarks in HepG2 (Figure 5a-c and S5c-d; Table

335 S3 and S9). In H3K4me3 highly enriched regions (top100), the sgRNA density is

$33643 \mathrm{sgRNAs}$ per $\mathrm{kb}$. The abundance distribution of sgRNAs inside H3K4me3 hallmarks

337 observes the pattern of their template DNAs (Figure 5b and S5c). We have identified

33814540 ssgRNAs $(75.82 \%$ are inside non-coding regions), which represent 6475

339 essential regulatory elements in HepG2 (Figure 5a-c and S5d; Table S4). ssgRNAs

340 appear in regions from weak to strong H3K4me3 elements, and the majority are in

341 strong elements regions (Figure 5b). Among all essential H3K4me3 elements 62.89\%

342 elements are targeted by 1 ssgRNA while $37.11 \%$ elements are targeted by multiple

343 ssgRNAs (Table S3). Detailed positions of ssgRNAs inside H3K4me3 elements can be

344 found in the Supplemental table 4, indicating the core regulatory sites of these essential

345 H3K4me3 elements. We also verified three randomly picked ssgRNAs targeting non-

346 coding regions, and all can significantly inhibit HepG2 growth (Figure 5d and S5e-f).

347 The H3K4me3 ssgRNAs evenly distribute on most chromosomes, with regions on

348 chromosome 5, 8 and 13 being exceptions, in which there is no shortage of H3K4me3

349 hallmarks and sgRNAs (Figure 5a). This indicates that fewer essential genes exist in 350 these regions. 
Pan et al 2020

351

352

353

354

355 Most H3K4me3 ssgRNAs locate on exons, proximal promoters, and introns (Figure S5d; Table S4). Normally, genes are more efficiently inactivated by mutations on their exons than their regulatory regions. Hence, the percentage of the ssgRNAs on exons is significantly greater than the percentage of the ssgRNAs on other regions (Figure S5d). The enriched GO terms of ssgRNA targeting exons are related to essential biological processes such as ncRNA metabolic and mitochondrial function, which play central roles in malignancy through macromolecular synthesis and energy production $^{43,44}$ (Figure S5g; Table S10), while the enriched GO terms of ssgRNAs targeting proximal promoters and UTRs are also related to the essential biological processes of cell survival (Figure 5e and S5g; Table S10).

\section{Annotation of the essential CTCF hallmarks in human liver cancer cells}

CTCF hallmarks stabilize mammalian genomes into discrete structural and regulatory domains, those of which can either prevent or facilitate the interactions of promoters and enhancers across their boundaries ${ }^{15}$. Although previous works substantiate that CTCF hallmarks can evolve in human cancers ${ }^{45}$, their underlying mechanisms are principally more difficult to define as a result of missing functional annotation of CTCF hallmarks in human.

To interrogate essential CTCF hallmarks in HepG2, we generated CTCF sgRNA libraries and performed the CRISPR-screening as described above (Figure 1a and 2a).

371 Overall, we screened $1.06 \mathrm{M}$ sgRNAs, targeting $79.75 \%$ of the CTCF hallmarks in 
HepG2 (Figure 6a-c and S6a-b; Table S3 and S11). In strong CTCF binding regions

373 (top100), the sgRNA density is 63sgRNAs per kb. As expected, the abundance

374 distribution of sgRNAs inside CTCF hallmarks is notably more even than that of

$375 \mathrm{H} 3 \mathrm{~K} 4 \mathrm{me} 3$, and the patterns of their template DNAs are observed all the while (Figure

$3766 \mathrm{~b}$ and 5b). We identified 4628 CTCF ssgRNAs which represent 3583 (44.63\% inside

377 intergenic regions) essential CTCF hallmarks for HepG2 growth (Figure S6b; Table

378 S4). ssgRNAs appear in regions from weak to strong CTCF elements (Figure 6b).

379 Among all HepG2 essential CTCF elements, $78.76 \%$ elements are targeted by 1

380 ssgRNA while $21.24 \%$ elements are targeted by multiple ssgRNAs (Table S3). Detailed

381 positions of ssgRNAs inside CTCF elements can be found in the Supplemental table 4.

382 We verified three randomly picked intergenic CTCF ssgRNAs. As expected, all can

383 significantly inhibit HepG2 growth (Figure 6d and S6c-d).

384 CTCF ssgRNAs on chromosomes roughly follow the distribution of their matrix

385 hallmarks and sgRNAs (Figure 6a). As we known that CTCF hallmarks stabilize

386 genome from smaller loops into huge megabase-sized loops called topologically

387 associated domains (TADs) ${ }^{46,47}$, CTCF ssgRNAs will disrupt the topological structure

388 at different levels and result in their uneven distribution on many regions (Figure 6a).

389 Major CTCF hallmarks exist on distal promoters, introns, and proximal promoters

390 in HepG2 (Figure S6b; Table S4). CTCF ssgRNA targeting introns and proximal

391 promoters have significant GO terms including signaling transduction and cell

392 morphogenesis, all of which are crucial for cancers (Figure 6e and S6e; Table S12). 
Pan et al 2020

393

394

395

396

397

398

399

400

401

402

403

404

405

406

407

408

409

410

411

412

413

Only a minor portion of CTCF ssgRNAs target exons (Figure S6b). The majority of

GO terms of expressed genes whose exons are targeted by ssgRNAs are related to essential biological processes (Figure S6f; Table S12). Because major functions of CTCF hallmarks on exons are beyond promoting their sitting genes expression ${ }^{15}$, the GO term significance is lesser than that of H3K4me3 ssgRNA targeting exons (Figure S6f and S5g; Table S12 and S10).

The majority of CTCF hallmarks in HepG2 are common (79.79\%) among 55 human cell types (Figure 6f; Table S13). As a tissue-specific cell line, the spatial chromosome structure of HepG2 has been adapted to the requirements of liver functions, and it is not necessary to keep so many spatial chromosome structures specific for other cell types. Unlike mESCs, the percentage of the cell-type specific essential CTCF hallmarks corresponds with the percentage of the cell-type specific CTCF hallmarks in HepG2 (Figure 6f and 4f).

\section{H3K4me3 hallmark-LINC00339-CDC42 axis maintaining HepG2 growth}

We believe that functional epigenome annotation can facilitate uncovering novel regulation mechanisms and biomarkers of cancer cells. We focused on a $\mathrm{H} 3 \mathrm{~K} 4 \mathrm{me} 3$ ssgRNA (chr1-22352881), which targets the intron of LINC00339 that is highly expressed in multiple cancer cell lines (Figure 7a). Because H3K4me3 activates local gene expression, we checked if ssgRNA (chr1-22352881) would disrupt LINC00339 expression. As predicted, the LINC00339 level is significantly decreased after ssgRNA 
414 (chr1-22352881) disruption (Figure 7b). Ensuing, we knocked down the expression of

415 LINC00339, and discovered that the proliferation of HepG2 is significantly inhibited

416 into the same level as ssgRNA (chr1-22352881) disruption (Figure 7c-e). Cell-cycle

417 analysis shows that both ssgRNA (chr1-22352881) disruption and knocking down

418 LINC00339 blocks the S-phase entry of HepG2 but not hESCs (Figure 7f). Altogether,

419 the data suggests that ssgRNA (chr1-22352881) disruption compromises HepG2

420 proliferation through downregulating the expression of LINC00339.

421 It has recently became apparent that long non-coding RNAs (lncRNAs) can

422 function as transcriptional activators ${ }^{48}$. Through binding to histone-modifying

423 complexes, transcription factors and RNA polymerase II, lncRNAs can promote gene

424 expression in cis or in $\operatorname{trans}^{48}$. Located at the immediate downstream of LINC00339,

425 CDC42 functions in cell-cycle and anchorage-independent growth (Figure S5f) ${ }^{49,50}$.

426 CDC42 also transduces growth and adhesion signals to drives cell-cycle progress from

427 G1 to S phase ${ }^{49,50}$. Therefore, LINC00339 may promote CDC42 expression in HepG2.

428 As expected, we found that both ssgRNA (chr1-22352881) disruption and knocking-

429 down LINC00339 significantly decreases the expression of CDC42 (Figure 7g-h). Our

430 data establishes that the H3K4me3 hallmark targeted by ssgRNA (chr1-22352881)

431 orchestrates the activity of LINC00339-CDC42 axis to promote HepG2 growth. 
433

434

435

436

437

438

439

440

441

442

443

444

445 Protospacer Adjacent Motif (PAM) ${ }^{51}$. Without PAM, around 70-77\% sgRNAs

446 generated by CORALINA are ineffective. Hiroshi Arakawa developed another

447 approach that employs six type IIs or type III restriction enzymes (EcoP15I, Bgl II, Acu

448 I, XbaI, Bsm BI and AatI) digestions and PAGE-Gel purifications to convert mRNA

449 into sgRNAs with flanking $\mathrm{PAM}^{52}$. Because six restriction enzymes digestions will

450 destroy $6.2 \%$ potential sgRNAs and multiple PAGE-Gel purification significantly

451 increases DNA loss, Arakawa's approach is not practicable for large-scale CRISPR-

452 screening. Our CTDE approach overcomes pervious limitations by generating sgRNA

453 libraries from source DNAs with a simple- and cost-effective procedure. 

sgRNA library is not practicable for whole transcriptome screening. been shown efficient discovery and functional characterization of regulatory elements ${ }^{21}$.

463 This type of approach needs more than three paired-guide RNAs for each hallmark, and

464 the library cost to cover all CTCF and H3K4me3 hallmarks (79K in mESCs and 69K

465 in HepG2) proves to be too costly. In addition, once asynchronous cutting happens, the

466 sequence of the first cutting site will change ${ }^{53}$ and the hallmark cannot be deleted by

467 the paired-guide RNAs anymore. So, the paired-guide RNA approach needs

468 CRISPR/Cas9 cut both sites simultaneously, which leads to a lower efficiency than

469 single sgRNA system. Thus, the paired-guide RNA library-based screening requires a

470 more sensitive readout than the survival readout used in this work.

471 The essential hallmarks datasets of H3K4me3 and CTCF in mESCs and HepG2 472 can provide potential benefits for multiple fields in the future. For instance, Induced 473 Pluripotent Stem Cells (iPSCs) researchers should evaluate the somatic mutations 474 inside the essential hallmarks of mESCs, which can significantly compromise the 
Pan et al 2020

475 reprogramming efficiency. Additionally, the H3K4me3 essential hallmarks related to 476 core transcriptional factors can be utilized to boost the reprogramming efficiency 477 through CRISPR mediated activation ${ }^{54}$. The HepG2 datasets permits cancer researchers 478 to direct their focus on $\mathrm{H} 3 \mathrm{~K} 4 \mathrm{me} 3$ or/and CTCF essential hallmarks, which are specific 479 in liver cancer cells. Through reversible or permanent inactivation of these hallmarks 480 via CRISPR mediated approaches, potential novel liver cancer treatment strategies can 481 be developed ${ }^{55}$. CTDE provides a simple, time- and cost-effective procedure to convert source 483 DNA to sgRNA library, which could be more broadly applied to functional epigenome 484 annotation in a biological context. 


\section{References}

488 1. TA., B. Genomes. 2nd edition.

489 2. Bonev, B. \& Cavalli, G. Organization and function of the 3D genome. Nat Rev Genet 17, 772

$490 \quad$ (2016).

491 3. Bernstein, B.E., Meissner, A. \& Lander, E.S. The mammalian epigenome. Cell 128, 669-681

492 (2007).

493 4. Abbott, A. Project set to map marks on genome. Nature 463, 596-597 (2010).

494 5. Consortium, E.P. et al. Identification and analysis of functional elements in $1 \%$ of the human 495 genome by the ENCODE pilot project. Nature 447, 799-816 (2007).

496 6. Boyle, A.P. et al. High-resolution mapping and characterization of open chromatin across the 497 genome. Cell 132, 311-322 (2008).

498 7. Kurdyukov, S. \& Bullock, M. DNA Methylation Analysis: Choosing the Right Method. Biology $499 \quad$ (Basel) 5 (2016).

500 8. Barski, A. et al. High-resolution profiling of histone methylations in the human genome. Cell $501 \quad$ 129, 823-837 (2007).

502 9. Sims, R.J., 3rd, Nishioka, K. \& Reinberg, D. Histone lysine methylation: a signature for chromatin 503 function. Trends Genet 19, 629-639 (2003).

504 10. Wysocka, J. et al. A PHD finger of NURF couples histone H3 lysine 4 trimethylation with 505 chromatin remodelling. Nature 442, 86-90 (2006).

506 11. Bernstein, B.E. et al. A bivalent chromatin structure marks key developmental genes in 507 embryonic stem cells. Cell 125, 315-326 (2006).

508 12. Phillips, J.E. \& Corces, V.G. CTCF: master weaver of the genome. Cell 137, 1194-1211 (2009).

509 13. Chaumeil, J. \& Skok, J.A. The role of CTCF in regulating V(D)J recombination. Curr Opin Immunol 24, 153-159 (2012).

511 14. Guelen, L. et al. Domain organization of human chromosomes revealed by mapping of nuclear lamina interactions. Nature 453, 948-951 (2008).

15. Kim, S., Yu, N.K. \& Kaang, B.K. CTCF as a multifunctional protein in genome regulation and gene

515 16. Ong, C.T. \& Corces, V.G. CTCF: an architectural protein bridging genome topology and function. Nat Rev Genet 15, 234-246 (2014).

517 17. Khoury, A. et al. Constitutively bound CTCF sites maintain 3D chromatin architecture and longrange epigenetically regulated domains. Nat Commun 11, 54 (2020).

519 18. Joung, J. et al. Genome-scale CRISPR-Cas9 knockout and transcriptional activation screening. $520 \quad$ Nat Protoc 12, 828-863 (2017).

521 19. Shalem, O., Sanjana, N.E. \& Zhang, F. High-throughput functional genomics using CRISPR-Cas9. $522 \quad$ Nat Rev Genet 16, 299-311 (2015).

523 20. Fulco, C.P. et al. Systematic mapping of functional enhancer-promoter connections with CRISPR $524 \quad$ interference. Science 354, 769-773 (2016).

525 21. Diao, Y. et al. A tiling-deletion-based genetic screen for cis-regulatory element identification in 526 mammalian cells. Nat Methods 14, 629-635 (2017).

527 22. Shah, S.A., Erdmann, S., Mojica, F.J. \& Garrett, R.A. Protospacer recognition motifs: mixed 528 identities and functional diversity. RNA Biol 10, 891-899 (2013). 
Pan et al 2020

529

530

531

532

533

534

535

536

537

538

539

540

541

542

543

544

545

546

547

548

549

550

551

552

553

554

555

556

557

558

559

560

561

562

563

564

565

566

567

568

569

570

23. Jinek, M. et al. A programmable dual-RNA-guided DNA endonuclease in adaptive bacterial immunity. Science 337, 816-821 (2012).

24. Sternberg, S.H., Redding, S., Jinek, M., Greene, E.C. \& Doudna, J.A. DNA interrogation by the CRISPR RNA-guided endonuclease Cas9. Nature 507, 62-67 (2014).

25. Hsu, P.D., Lander, E.S. \& Zhang, F. Development and applications of CRISPR-Cas9 for genome engineering. Cell 157, 1262-1278 (2014).

26. Fu, Y., Sander, J.D., Reyon, D., Cascio, V.M. \& Joung, J.K. Improving CRISPR-Cas nuclease specificity using truncated guide RNAs. Nat Biotechnol 32, 279-284 (2014).

27. Ran, F.A. et al. Double nicking by RNA-guided CRISPR Cas9 for enhanced genome editing specificity. Cell 154, 1380-1389 (2013).

28. Metzker, M.L. et al. Termination of DNA synthesis by novel 3'-modified-deoxyribonucleoside 5'-triphosphates. Nucleic Acids Res 22, 4259-4267 (1994).

29. Gibson, D.G. et al. Enzymatic assembly of DNA molecules up to several hundred kilobases. Nat Methods 6, 343-345 (2009).

30. Bodapati, S., Daley, T.P., Lin, X., Zou, J. \& Qi, L.S. A benchmark of algorithms for the analysis of pooled CRISPR screens. Genome Biol 21, 62 (2020).

31. Li, W. et al. MAGeCK enables robust identification of essential genes from genome-scale CRISPR/Cas9 knockout screens. Genome Biol 15, 554 (2014).

32. Bracewell, R. The Fourier Transform \& Its Applications 3rd Edition. Book.

33. Diao, Y. et al. A new class of temporarily phenotypic enhancers identified by CRISPR/Cas9mediated genetic screening. Genome Res 26, 397-405 (2016).

34. Weake, V.M. \& Workman, J.L. Inducible gene expression: diverse regulatory mechanisms. Nat Rev Genet 11, 426-437 (2010).

35. Malik, S. \& Roeder, R.G. The metazoan Mediator co-activator complex as an integrative hub for transcriptional regulation. Nat Rev Genet 11, $761-772$ (2010).

36. Ong, C.T. \& Corces, V.G. Enhancer function: new insights into the regulation of tissue-specific gene expression. Nat Rev Genet 12, 283-293 (2011).

37. Kim, T.H. et al. Analysis of the vertebrate insulator protein CTCF-binding sites in the human genome. Cell 128, 1231-1245 (2007).

38. Dixon, J.R. et al. Chromatin architecture reorganization during stem cell differentiation. Nature 518, 331-336 (2015).

39. Cuddapah, S. et al. Global analysis of the insulator binding protein CTCF in chromatin barrier regions reveals demarcation of active and repressive domains. Genome Res 19, 24-32 (2009).

40. Blum, A., Wang, P. \& Zenklusen, J.C. SnapShot: TCGA-Analyzed Tumors. Cell 173, 530 (2018).

41. Consortium, I.T.P.-C.A.o.W.G. Pan-cancer analysis of whole genomes. Nature 578, 82-93 (2020).

42. Rheinbay, E. et al. Analyses of non-coding somatic drivers in 2,658 cancer whole genomes. Nature 578, 102-111 (2020).

43. Zong, W.X., Rabinowitz, J.D. \& White, E. Mitochondria and Cancer. Mol Cell 61, 667-676 (2016).

44. Anastasiadou, E., Jacob, L.S. \& Slack, F.J. Non-coding RNA networks in cancer. Nat Rev Cancer 18, 5-18 (2018).

45. Song, S.H. \& Kim, T.Y. CTCF, Cohesin, and Chromatin in Human Cancer. Genomics Inform 15, 114-122 (2017). 
Pan et al 2020

571 46. Yu, M. \& Ren, B. The Three-Dimensional Organization of Mammalian Genomes. Annu Rev Cell

$572 \quad$ Dev Biol 33, 265-289 (2017).

573 47. Pombo, A. \& Dillon, N. Three-dimensional genome architecture: players and mechanisms. Nat

$574 \quad$ Rev Mol Cell Biol 16, 245-257 (2015).

575 48. Long, Y., Wang, X., Youmans, D.T. \& Cech, T.R. How do IncRNAs regulate transcription? Sci Adv

$576 \quad 3$, eaao2110 (2017).

577 49. Chou, M.M., Masuda-Robens, J.M. \& Gupta, M.L. Cdc42 promotes G1 progression through p70

578 S6 kinase-mediated induction of cyclin E expression. J Biol Chem 278, 35241-35247 (2003).

579 50. Qadir, M.I., Parveen, A. \& Ali, M. Cdc42: Role in Cancer Management. Chem Biol Drug Des 86,

$580 \quad 432-439$ (2015).

581 51. Koferle, A. et al. CORALINA: a universal method for the generation of gRNA libraries for CRISPR-

582 based screening. BMC Genomics 17, 917 (2016).

583 52. Arakawa, H. A method to convert mRNA into a gRNA library for CRISPR/Cas9 editing of any $584 \quad$ organism. Sci Adv 2, e1600699 (2016).

585 53. Sander, J.D. \& Joung, J.K. CRISPR-Cas systems for editing, regulating and targeting genomes.

587 54. Konermann, S. et al. Genome-scale transcriptional activation by an engineered CRISPR-Cas9

589 55. Qi, L.S. et al. Repurposing CRISPR as an RNA-guided platform for sequence-specific control of 590 gene expression. Cell 152, 1173-1183 (2013).

591

592

593 
Figure 1

a 1. Controlled template-dependent elongation

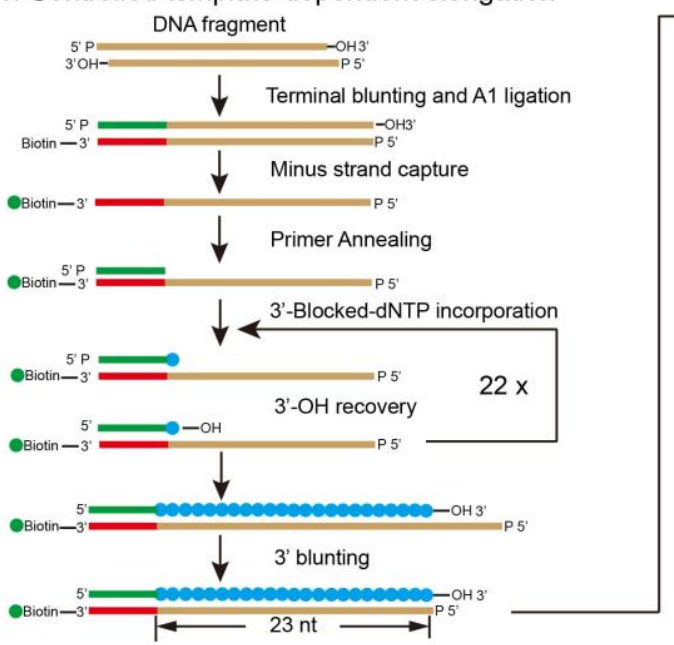

2. PAM selection and removal

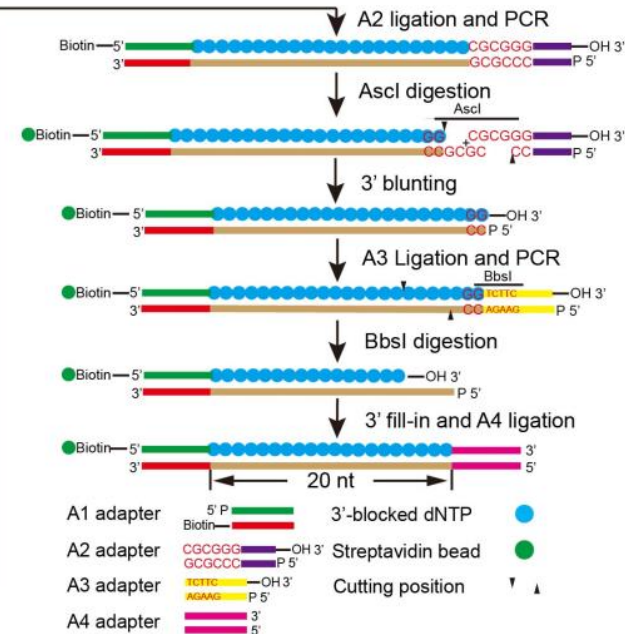

b
30

$(1.53 \%)$
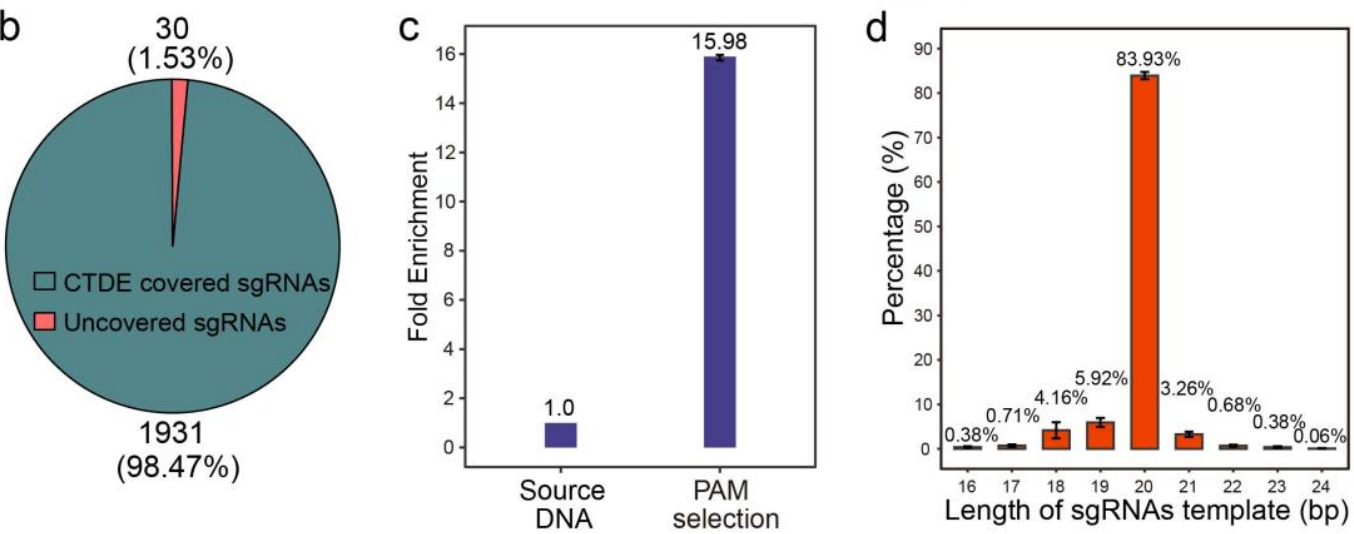

e

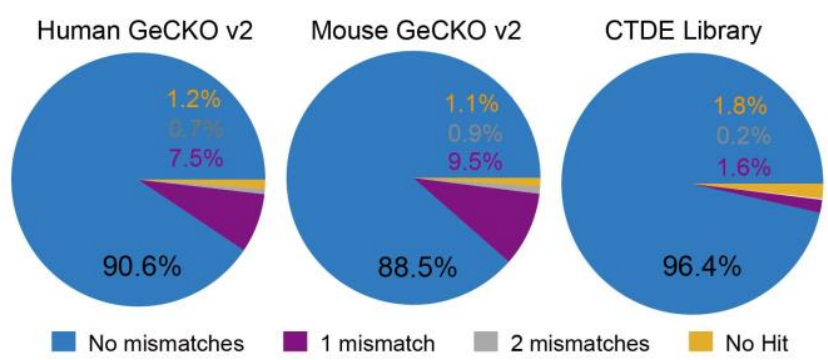

f

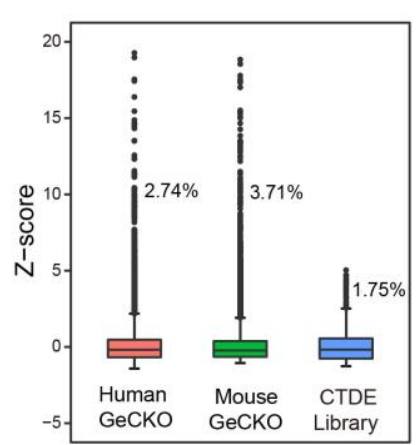

Figure 1 Controlled template-dependent elongation (CTDE) converts source DNA to a sgRNA

597 (a) Detailed schematics of the CTDE procedure. Briefly, sgRNA templates (23bp) are generated from fragmented input DNA by controlled template-dependent elongation; sgRNA templates with

599 NGG triplet in $3^{\prime}$ terminus are enriched and NGG triplets are removed before sgRNA templates are 600 cloned into a vector.

601 (b) The coverage rate of the potential sgRNA templates on source DNA (lentiCRISPR-V2) by CTDE.

602 (c) Fold enrichment of the sgRNA templates after NGG-PAM selection step. Error bars SD of 603 triplicates. 
Pan et al 2020

604

(d) Length distribution of the sgRNA templates from source DNA (lentiCRISPR-V2). Error bars

605 SD of triplicates.

606

(e) The fidelity of sgRNAs generated by CBDS (GeCKO libraries) and CTDE approaches; the

607 mapping quality of the sgRNAs against their targeting sites is used to show the library fidelity.

608 (f) The abundance bias of sgRNAs of CBDS (GeCKO libraries) and CTDE approaches; distribution

609 of sgRNA counts z-score of two GeCKO libraries (red and green) and CTDE library (blue) are 610 displayed.

611 
Figure 2

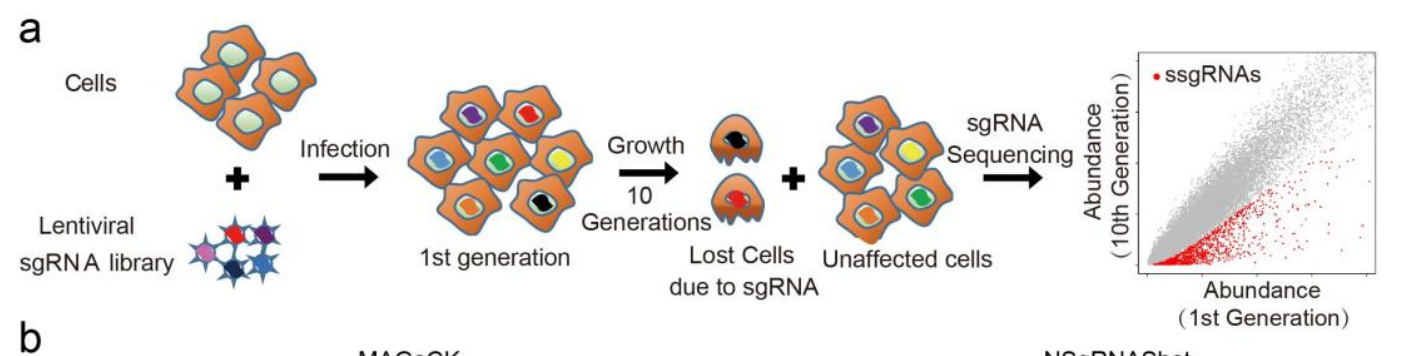

b
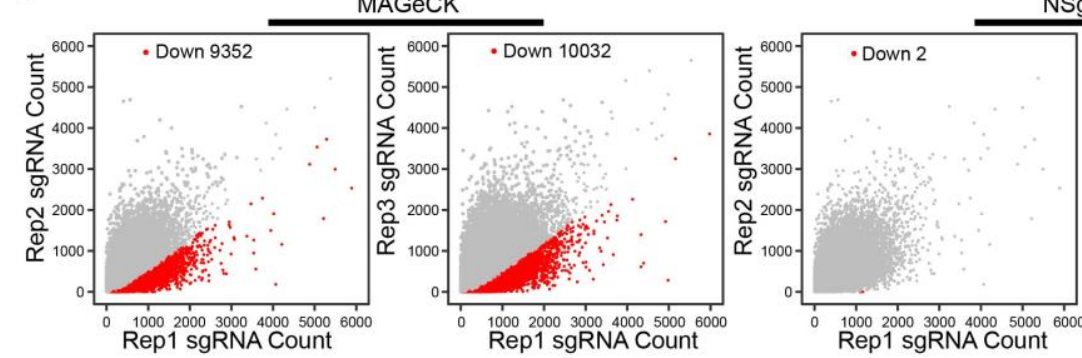

SgRNAShot

C
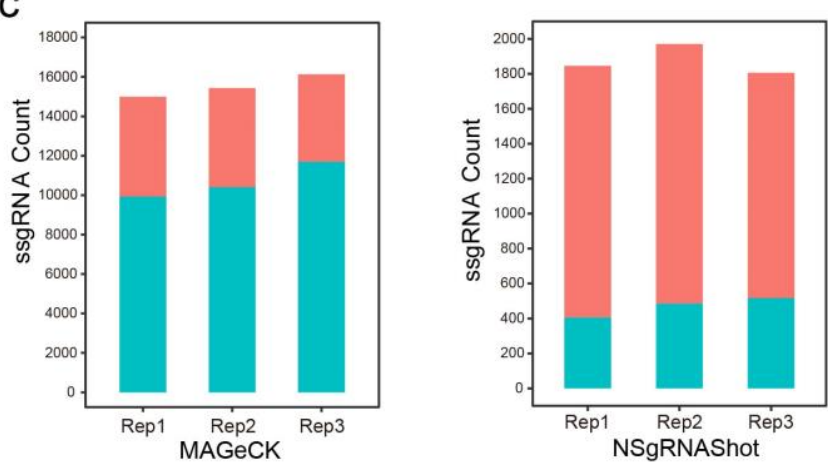

Essential gene Non-Essential gene

d
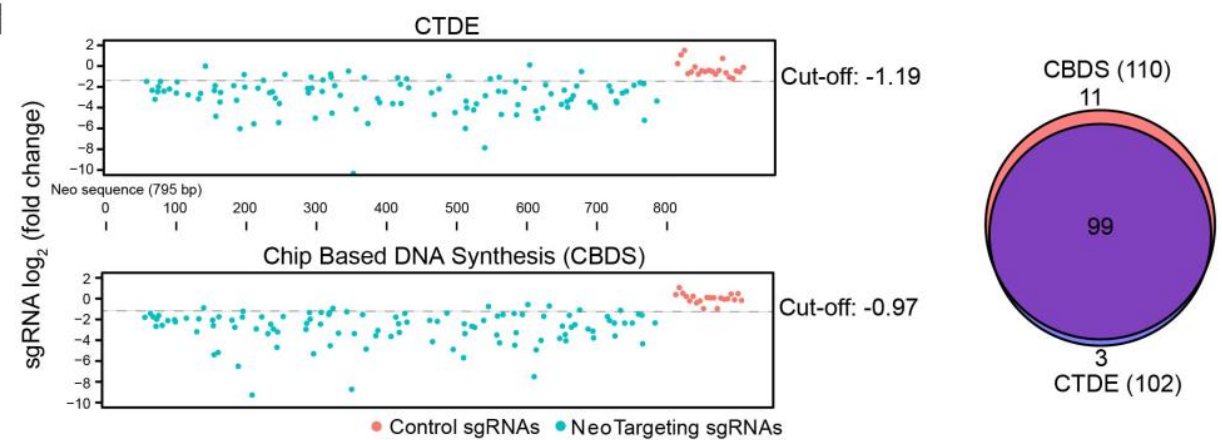

Figure 2 Validations of CTDE sgRNA library screening

(a) The schematic of CRISPR/Cas9 dropout screen: Cells are infected by library virus at low MOI; infected cells are cultured 10 generations; by comparing the abundance of sgRNA templates between the 1st and 10th generation, the sgRNAs which limit cell proliferation are identified by NSgRNAShot as significant sgRNAs(ssgRNAs).

(b) Comparison of false-positive rate of significantly changed sgRNA (ssgRNA) calling between MAGeCK and NSgRNAShot. Datasets of three biological replicates from an CRISPR dropout screen project (Tzelepis et al., 2016) are used (details in method). Scatter-plots exhibit sgRNA abundance at same time point between two biological replicate datasets. MAGeCK identifies 
Pan et al 2020

623 noticeably more false-positive ssgRNAs (red dots); NSgRNAShot identifies few false-positive 624 ssgRNAs.

625 (c) Bar plots display the number and distribution (essential genes and non-essential genes) of 626 ssgRNAs identified by MAGeCK and NSgRNAShot from above (b) CRISPR dropout screen 627 project.

628 (d) Left panel: $\log _{2}$ fold change of the sgRNA abundance of Neo gene targeting libraries generated 629 by CBDS and CTDE during validation screening (details in method). Red dots are non-targeting 630 control sgRNAs; green dots are Neo targeting sgRNAs. Right panel: the overlap of significantly 631 changed sgRNA (ssgRNA) from CBDS library and CTDE library.

632

633

634 


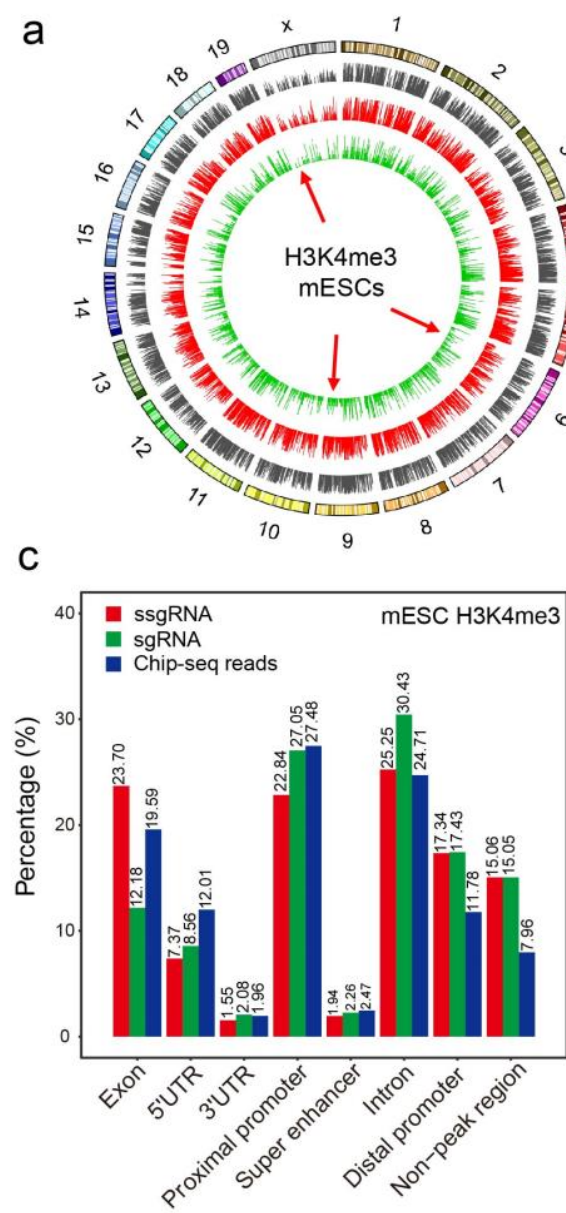

Figure 3

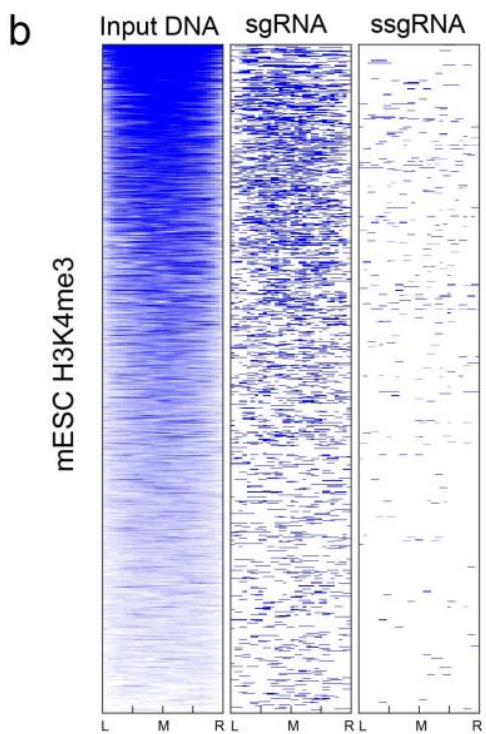

$f$

CDS targeted by sgRNA CDS targeted by ssgRNA

d

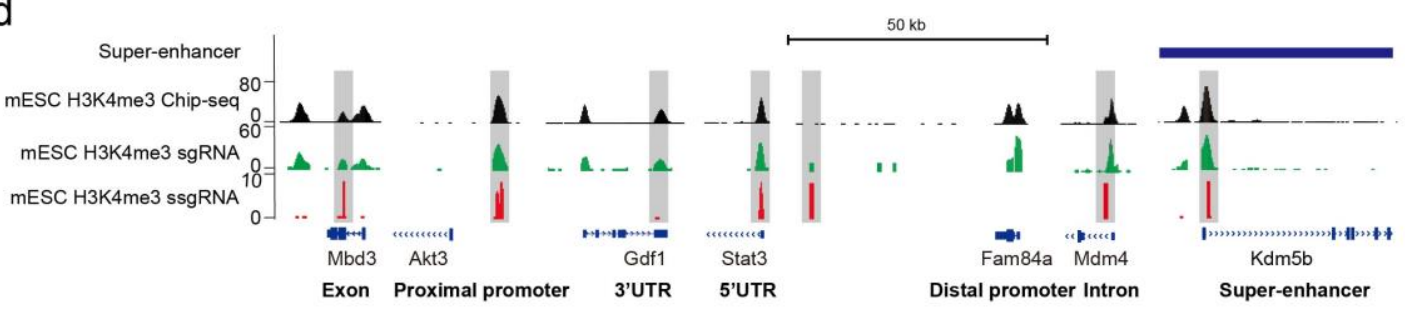

e
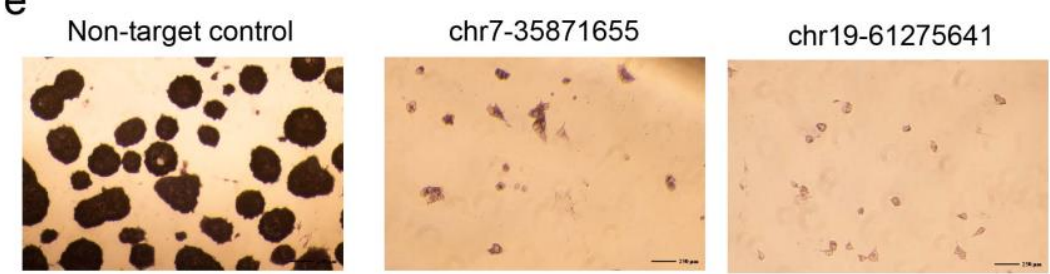

chr12-86827179

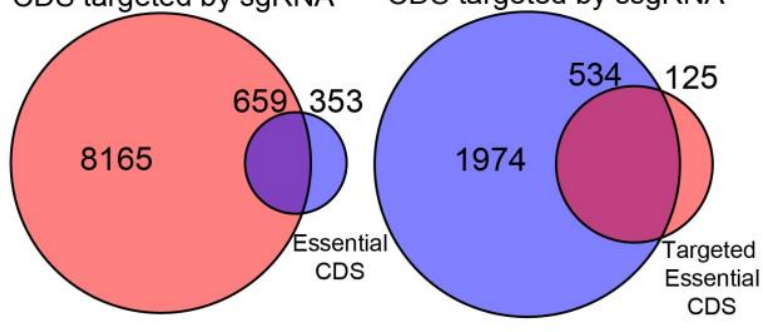

Figure 3 Annotation of the essential H3K4me3 hallmarks in mESCs self-renewal

(a) The circos plot of H3K4me3 ChIP-seq, H3K4me3 sgRNAs and H3K4me3 ssgRNAs profiles on mouse genome. The circles, from exterior to interior, illustrate mouse genome (mm9), ChIP-Seq reads density (black), H3K4me3 sgRNAs density (red) and H3K4me3 ssgRNAs density (green).

641 Arrows show regions with biased ssgRNAs distribution.

642 (b) Heat-map of input DNA, sgRNA and ssgRNA enrichment inside mESCs H3K4me3 elements.

643 All H3K4me3 elements are displayed in relative scale. L: Left boundary; M: Middle; R: Right 644 boundary. The heat-maps are rank-ordered according to the enrichment of input DNA (blue, 
Pan et al 2020

645 enriched; white, not enrichment).

646 (c) The distribution of H3K4me3 ChIP-seq reads, H3K4me3 sgRNAs and H3K4me3 ssgRNAs on

647 major regulatory regions of mouse genome.

648 (d) Plots of H3K4me3 ChIP-seq reads, H3K4me3 sgRNAs and H3K4me3 ssgRNAs at seven 649 genomic loci. Y-axes, RPKM. Genomic regions with enriched H3K4me3 ssgRNA are shaded grey.

650 (e) Validation of three H3K4me3 ssgRNA in mESCs self-renewal. Alkaline phosphatase (AP)

651 staining illustrates that three randomly selected H3K4me3 ssgRNAs significantly inhibit mESCs

652 self-renewal. ssgRNA is named by three factors (chromosome number; targeting strain + or -;

653 mapping position). Scale bar, $250 \mu \mathrm{m}$.

654 (f) Left panel: CDS targeted by mESCs H3K4me3 sgRNAs includes 659 well-recognized essential 655 genes (Shohat and Shifman, 2019; Tzelepis et al., 2016). Right panel: CDS targeted by mESCs

656 H3K4me3 ssgRNAs includes 534 genes from above 659 essential genes.

657

658 
Figure 4

a

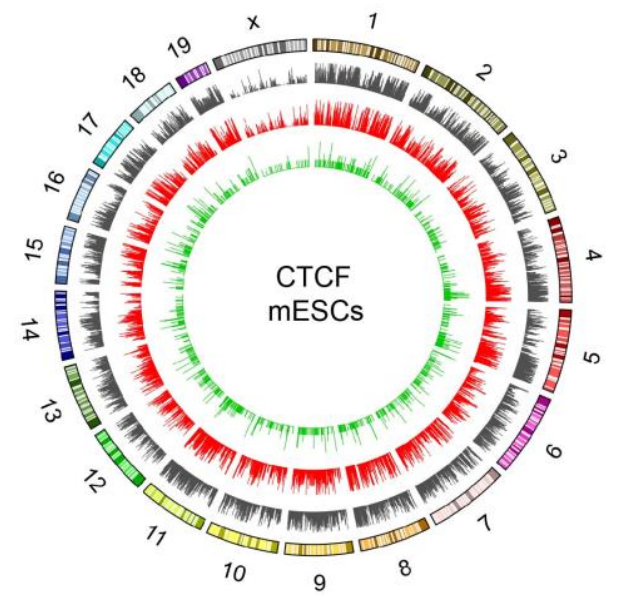

b

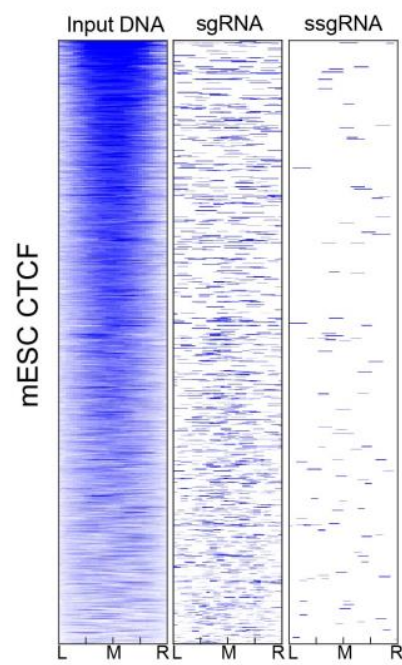

C mESCs

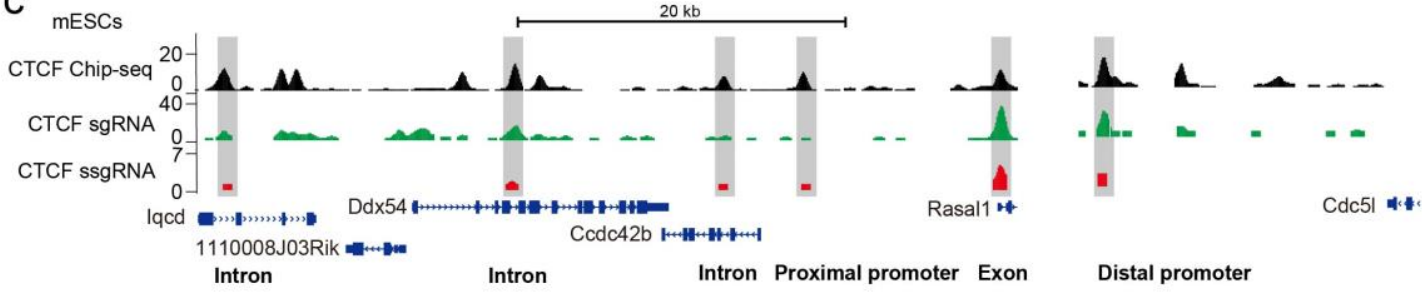

d Non-targe-control

chr19-21447441

chr15-12027231

chr14-63078202
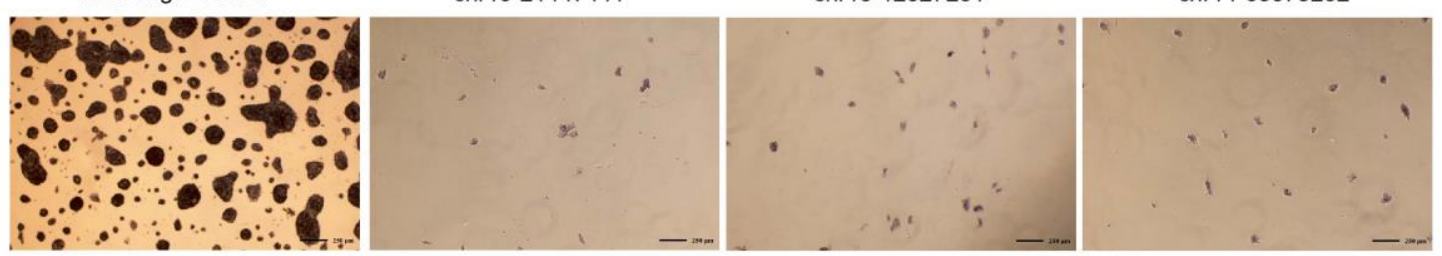

e
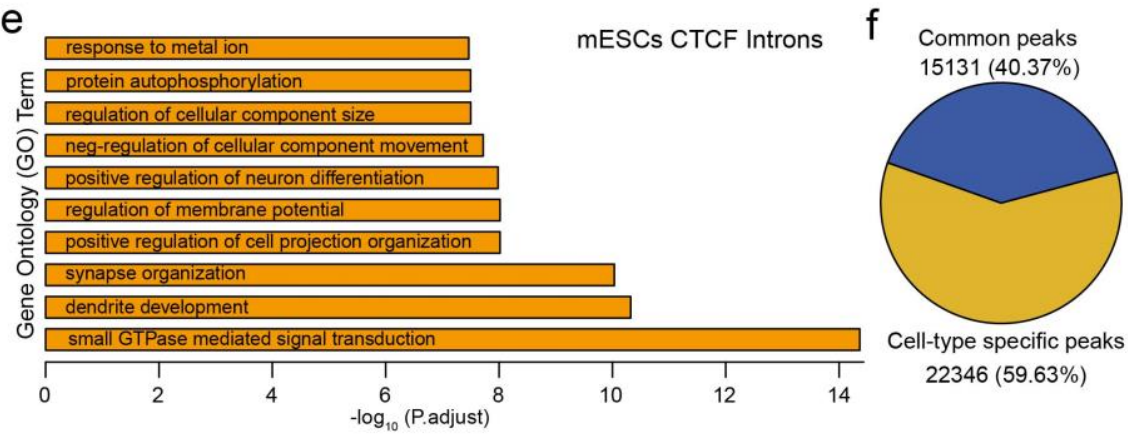

Essential common peaks $1887(71.15 \%)$



cell-type specific peaks $765(28.85 \%)$

(a) The circos plot of CTCF ChIP-seq, CTCF sgRNAs and CTCF ssgRNAs profiles on mouse genome. The circles, from exterior to interior, illustrate mouse genome (mm9), CTCF ChIP-Seq reads density (black), CTCF sgRNAs density(red) and CTCF ssgRNAs density (green).

(b) Heat-map of input DNA, sgRNA and ssgRNA enrichment inside mESCs CTCF elements. All

666 CTCF elements are displayed in relative scale. L: Left boundary; M: Middle; R: Right boundary.

667 The heat-maps are rank-ordered based on the enrichment of input DNA as follows: blue, enriched; white, not enrichment. 
(c) Plots of CTCF ChIP-seq reads, CTCF sgRNAs and CTCF ssgRNAs at six genomic loci. Y-axes,

670 RPKM. Genomic regions with CTCF ssgRNA are shaded grey.

671 (d) Validation of three CTCF ssgRNA in mESCs self-renewal. Alkaline phosphatase (AP) staining

672 shows that three randomly selected CTCF ssgRNAs significantly inhibit mESCs self-renewal.

673 ssgRNA is named by three factors (chromosome number; targeting strain + or -; mapping position).

674 Scale bar, $250 \mu \mathrm{m}$.

675 (e) Top 10 GO terms of the genes whose introns are targeted by CTCF ssgRNAs.

676 (f) Cell-type specific analysis of essential CTCF hallmark (targeted by ssgRNA) in mESCs self-

677 renewal. Major part (71.15\%) of essential CTCF hallmark in mESCs self-renewal are common VS

678 major part (59.63\%) of CTCF hallmarks in mESCs are cell-type specific.

679 
Figure 5

a

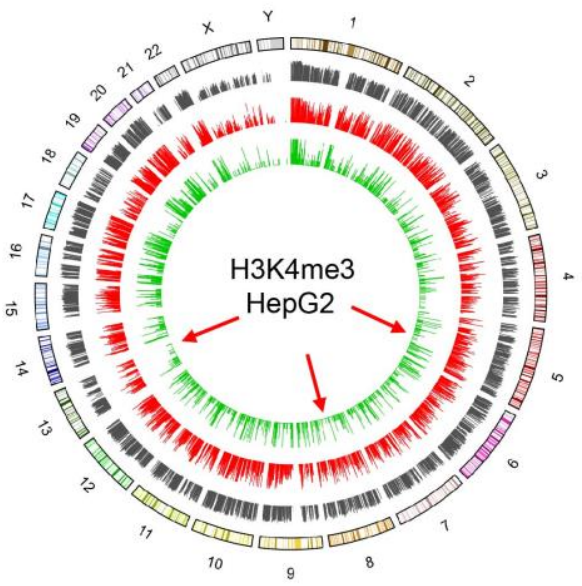

b

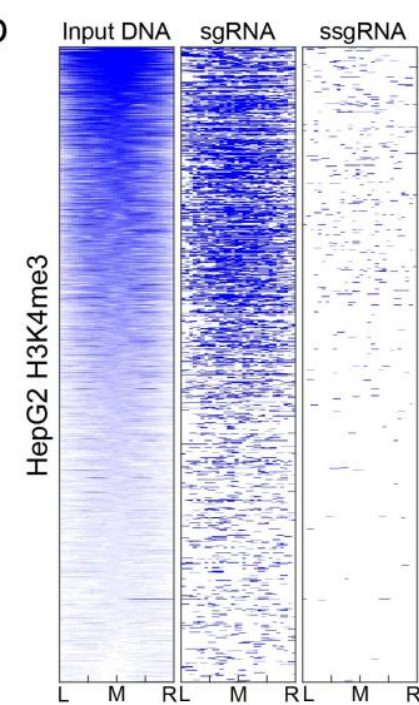

C

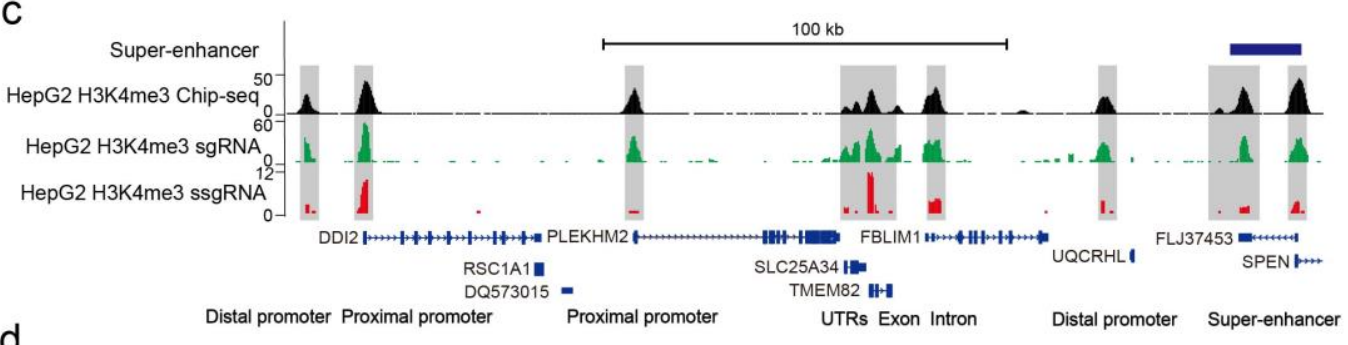

d

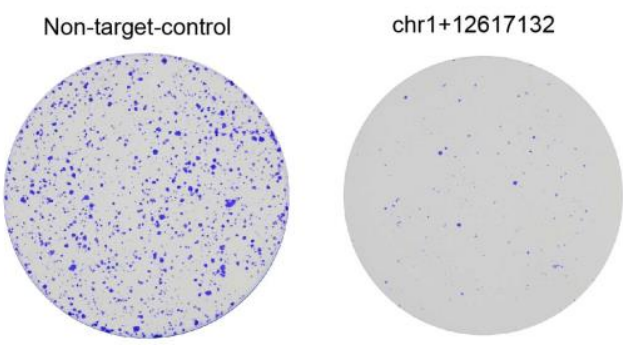

e

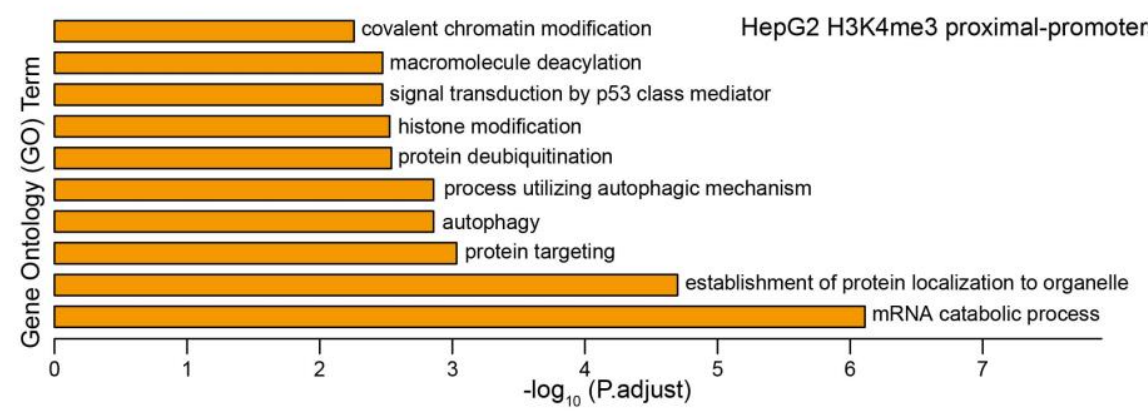
human genome. The circles, from exterior to interior, illustrate human genome (hg19), ChIP-Seq reads density (black), H3K4me3 sgRNAs density(red) and H3K4me3 ssgRNAs density (green). Arrows show regions with biased ssgRNAs distribution.

687 (b) Heat-map of input DNA, sgRNA and ssgRNA enrichment inside HepG2 H3K4me3 elements.

688 All H3K4me3 elements are displayed in relative scale. L: Left boundary; M: Middle; R: Right boundary. The heat-maps are rank-ordered based on the enrichment of input DNA as follows: blue, 
Pan et al 2020

690

enriched; white, not enrichment.

691 (c) Plots of H3K4me3 ChIP-seq reads, H3K4me3 sgRNAs and H3K4me3 ssgRNAs at eight 692 genomic loci. Y-axes, RPKM. Genomic regions with enriched H3K4me3 ssgRNA are shaded grey.

693 (d) Validation of three H3K4me3 ssgRNAs in HepG2. Crystal violet staining shows that three 694 randomly selected H3K4me3 ssgRNAs significantly inhibit HepG2 proliferation. ssgRNA is named 695 by three factors (chromosome number; targeting strain + or -; mapping position). Scale bar, $500 \mu \mathrm{m}$ 696 (e) Top 10 GO terms of the genes whose proximal promotors are targeted by H3K4me3 ssgRNAs.

697

698

699

700 
Figure 6

a

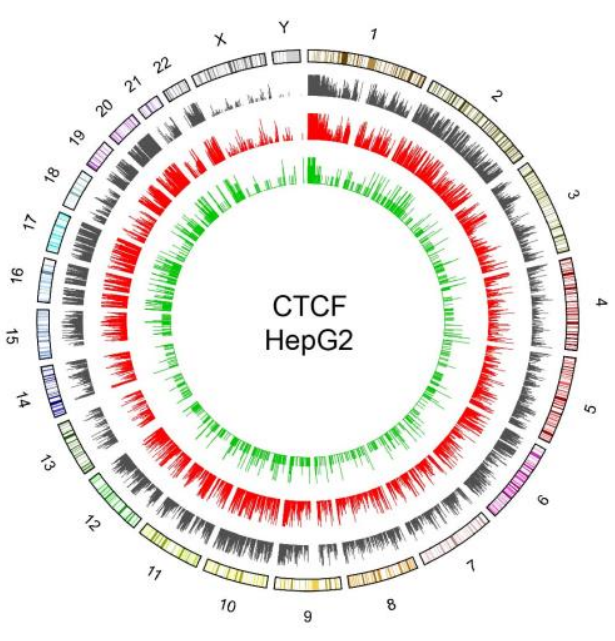

b

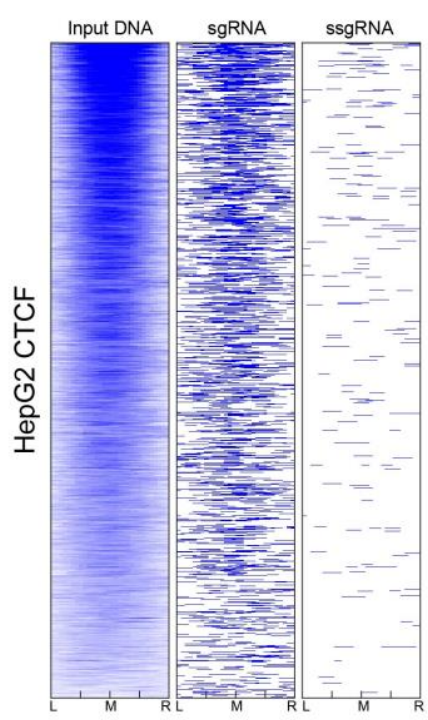

C

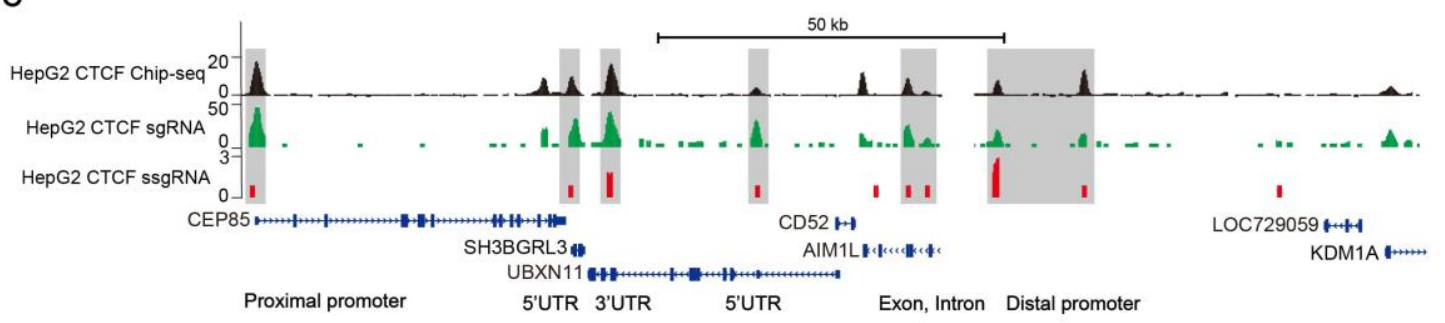

d

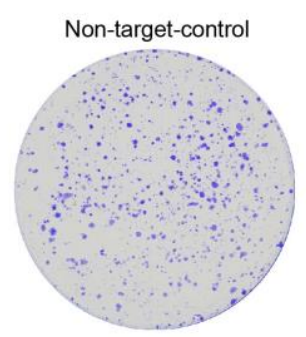

chr11-41682097

chr2-84542118

chr2+114361824

e

HepG2 CTCF Introns
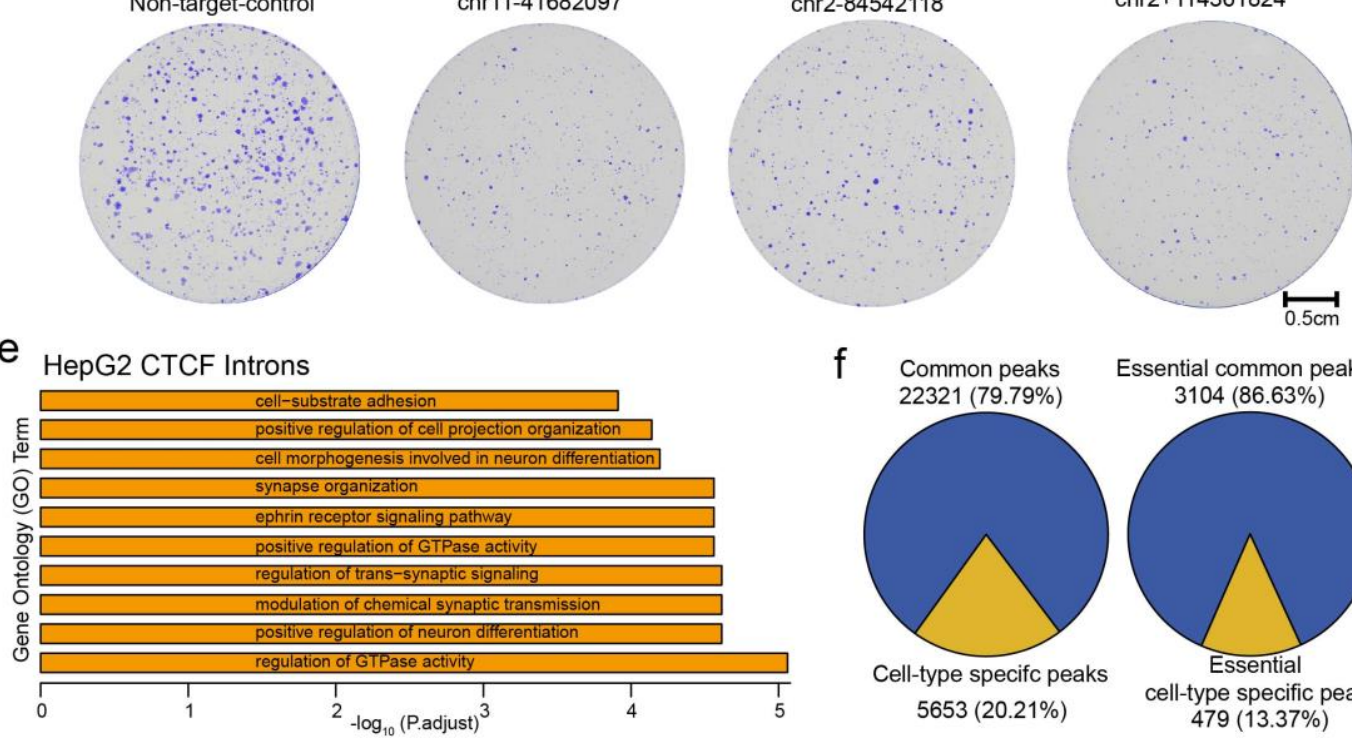

Essential common peaks

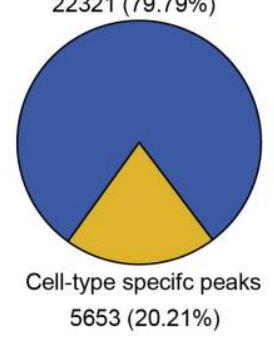
$3104(86.63 \%)$

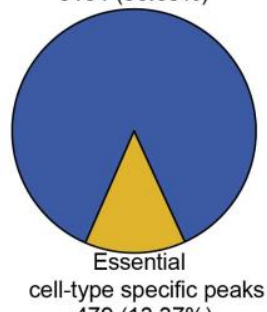

$479(13.37 \%)$

Figure 6 Annotation of the essential CTCF hallmarks in HepG2

(a) The circos plot of CTCF ChIP-seq, CTCF sgRNAs and CTCF ssgRNAs profiles on human genome. The circles, from outside to inside, illustrate human genome (hg19), CTCF ChIP-Seq reads density (black), CTCF sgRNAs density(red) and CTCF ssgRNAs density (green).

(b) Heat-map of input DNA, sgRNA and ssgRNA enrichment inside HepG2 CTCF elements. All

708 CTCF elements are displayed in relative scale. L: Left boundary; M: Middle; R: Right boundary. The heat-maps are rank-ordered based on the enrichment of input DNA as follows: blue, enriched; white, not enrichment. 
Pan et al 2020

711 (c) Plots of CTCF ChIP-seq reads, CTCF sgRNAs and CTCF ssgRNAs at seven genomic loci. Y712 axes, RPKM. Genomic regions with CTCF ssgRNA are shaded grey. (d) Validation of three CTCF ssgRNAs in HepG2 proliferation. Crystal violet staining illustrates that three randomly selected CTCF ssgRNAs significantly inhibit HepG2 proliferation. ssgRNA is named by three factors (chromesome number; targeting strain + or -; mapping position). Scale bar, $716500 \mu \mathrm{m}$

717 (e) Top 10 GO terms of the genes whose intron are targeted by CTCF ssgRNAs.

718 (f) Cell-type specific analysis of essential CTCF hallmark (targeted by ssgRNA) in HepG2 719 proliferation. Major part of both CTCF hallmarks (79.79\%) and essential CTCF hallmarks (86.63\%) 720 are common. 
Figure 7

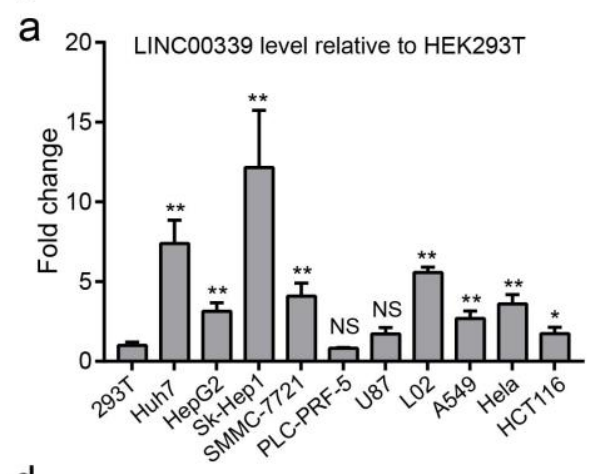

d

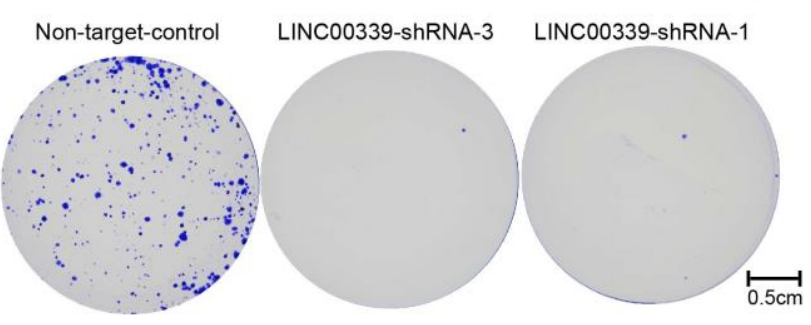

b

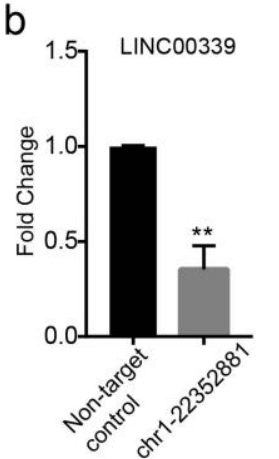

C

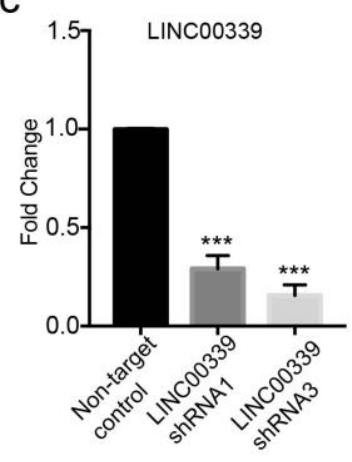

e

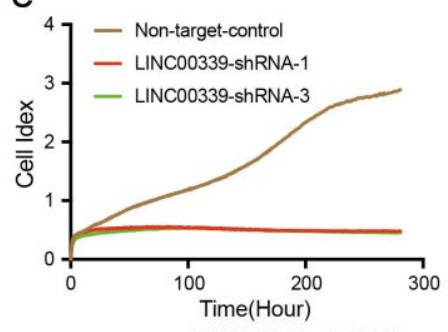

f
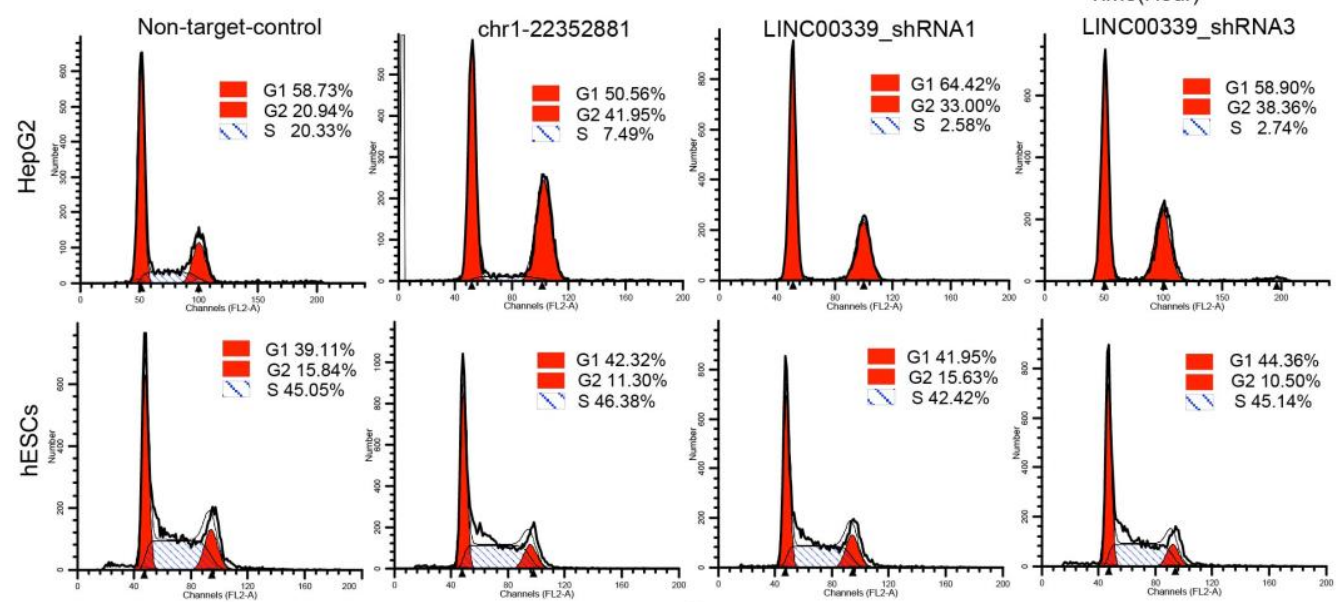

g

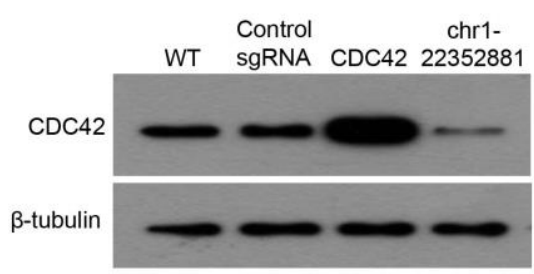

h

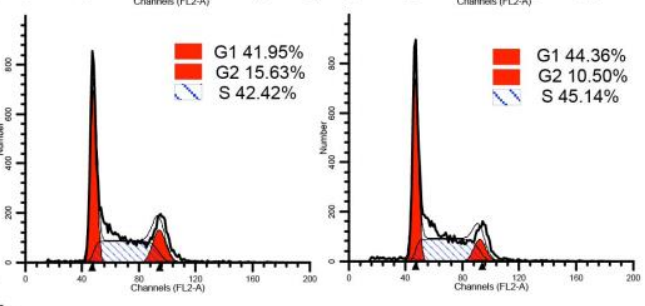

Figure 7 H3K4me3 hallmark-LINC00339-cdc42 axis maintaining HepG2 growth

(a) LINC00339 expression in ten human cancer cell lines by RT-PCR. Most human cancer cell lines have more LINC00339 than HEK293T. Student's T-test, ${ }^{* *} \mathrm{p}<0.001,{ }^{* *} \mathrm{p}<0.01$. Error bars SD of triplicates.

(b) Quantification of LINC00339 RNA level by RT-PCR after ssgRNA (chr1-22352881) disruption. ssgRNA (chr1-22352881) disruption significantly decreases LINC00339 level in HepG2. Student's

732 T-test, $* * * p<0.01$. Error bars SD of triplicates.

733 (c) Quantification of LINC00339 RNA level by RT-PCR after shRNA knockdown. Two shRNAs 
Pan et al 2020

734 can significantly decrease LINC00339 level in HepG2. Student's T-test, *** $\mathrm{p}<0.001$. Error bars SD 735 of triplicates.

736 (d) Proliferation assay of HepG2 after knockdown LINC00339. Knockdown LINC00339

737 significantly inhibits HepG2 proliferation. 14 days culture after LINC00339 knockdown, cells were 738 stained by crystal violet.

739 (e) Knockdown LINC00339 significantly inhibits HepG2 proliferation. Real-time cell numbers are 740 plotted.

741 (f) Flow cytometry analysis of HepG2 and hESCs after ssgRNA (chr1-22352881) disruptio and 742 LINC00339 knockdown. ssgRNA (chr1-22352881) disruption and LINC00339 knockdown only 743 block S-phase entry in HepG2.

744 (g) Western blot analysis of CDC42 after ssgRNA (chr1-22352881) disruption in HepG2. CDC42 745 over-expression and a scramble sgRNA as controls. Loading control, tubulin.

746 (h) Quantification of CDC42 mRNA by RT-PCR after LINC00339 knockdown. Student's T-test, $747 * * * \mathrm{p}<0.001$. Error bars SD of triplicates. 


\section{Figure $\mathrm{S} 1$}

a

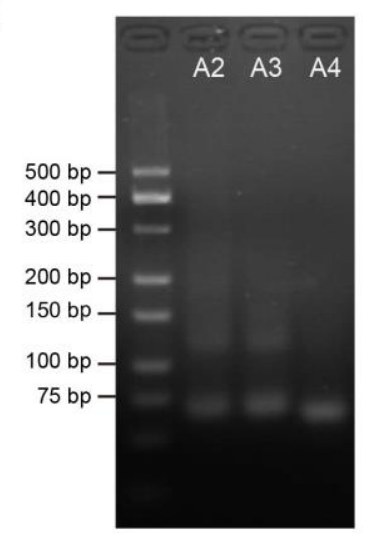

b

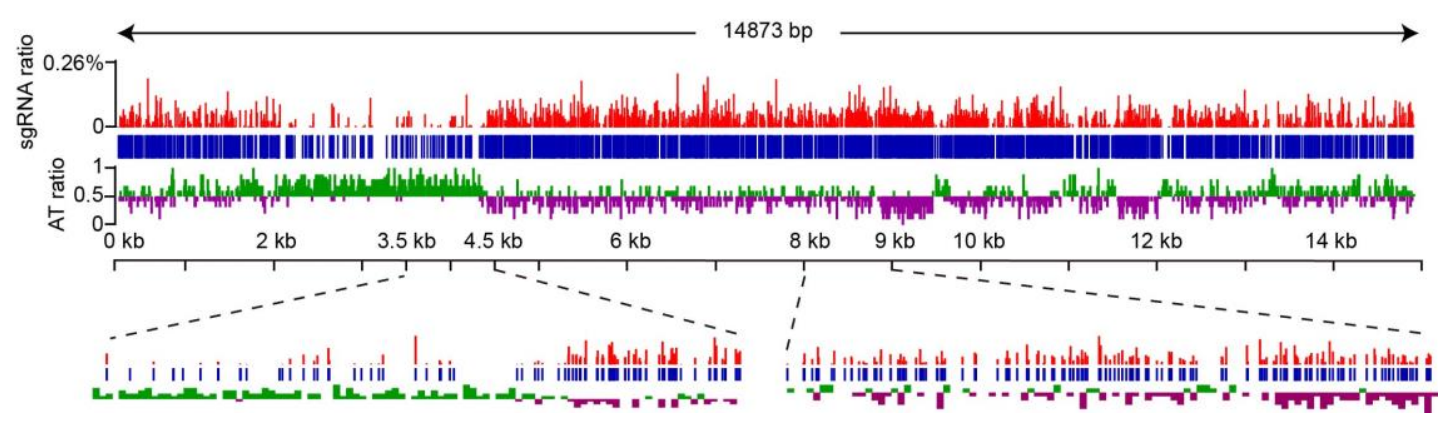

(a) Agarose gel (3.5\%) image of PCR product after adapter (A2, A3 and A4) ligation.

(b) The abundance distribution of sgRNA templates generated by CTDE on source DNA (LentiCRISPR-V2). Up panel: Red displays the relative abundance of sgRNA templates on each PAM (NGG) site. Middle: blue represents is potential sgRNA sites (NGG PAM) on source DNA. Down panel: The green and purple are the relative abundance of $\mathrm{A} / \mathrm{T}$ in $10 \mathrm{bp}$ bins (details in method).

759 A A/T-rich region and a balanced nucleotide region are zoomed in. 
Figure S2

a

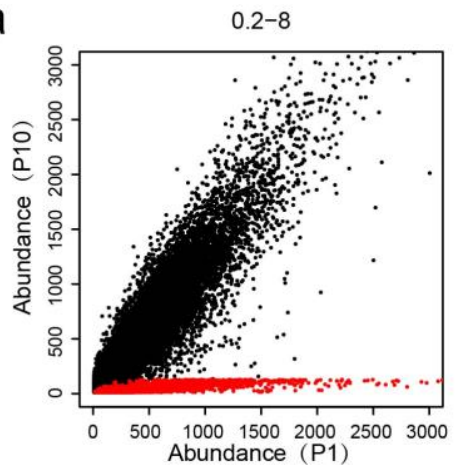

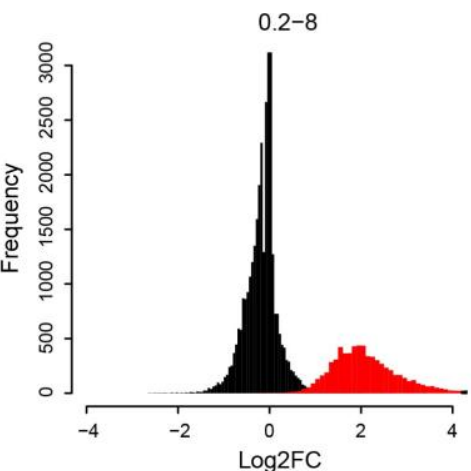

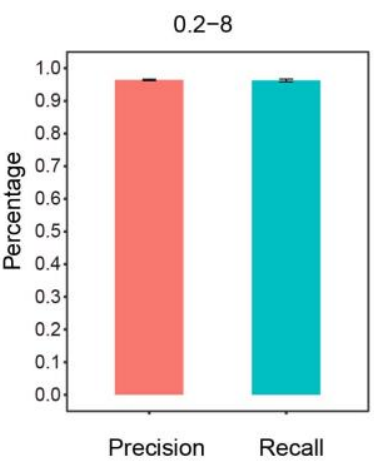

b

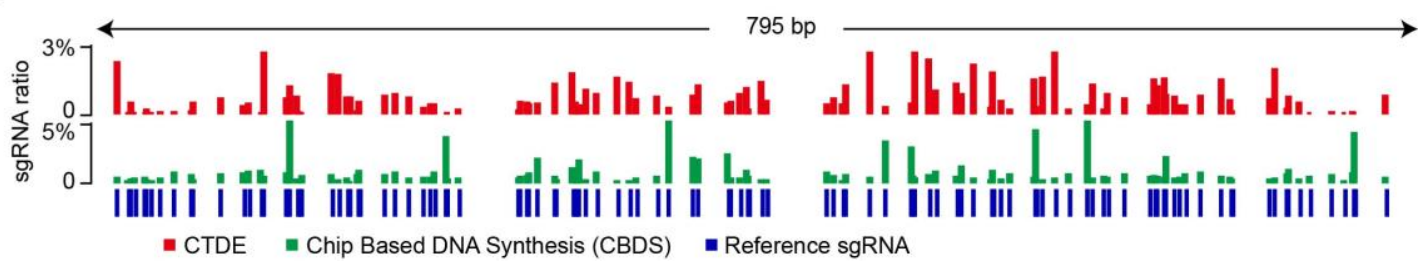

Figure S2

(a) NSgRNAShot can efficiently identify ssgRNA from a typical $(\alpha=0.2 ; \beta=8$; details in method) simulated dataset. Left picture: Scatter-plot of normalized read counts of sgRNAs at P1 and P10 of the simulated dataset. Middle picture: the abundance fold-change (FC) distribution of the sgRNAs. Right picture: the precision rate and the recall rate of NsgRNAShot in simulated dataset. The black represents the sgRNAs without suffering dropout. The red represents the sgRNAs suffering dropout. (b) The abundance distribution of sgRNA templates generated by CTDE and CBDS on Neo gene. Red displays the relative abundance of sgRNA templates (CTDE) on each PAM (NGG) site; green is the relative abundance of sgRNA templates (CBDS) on each PAM (NGG) site; blue shows potential sgRNA sites (NGG PAM) on Neo gene. 
Figure S3
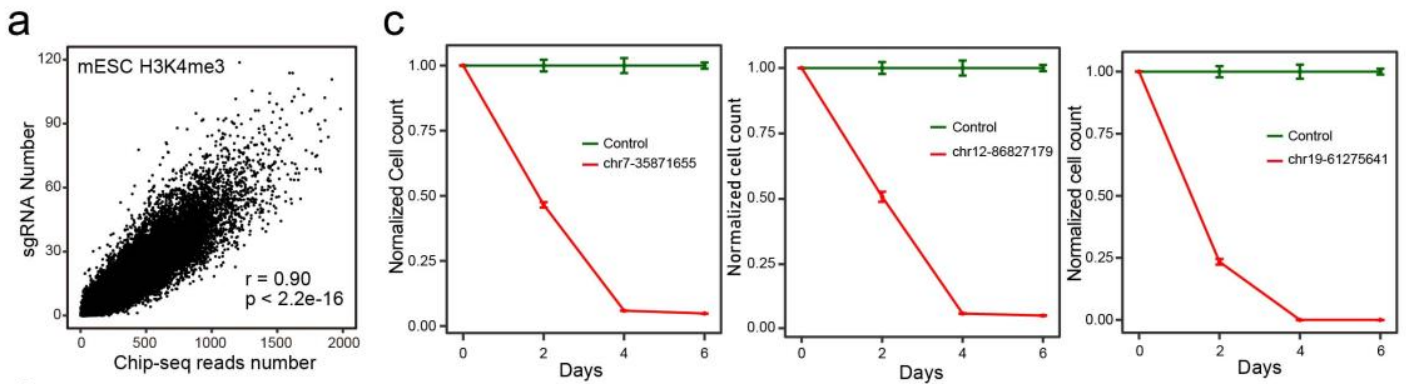

b

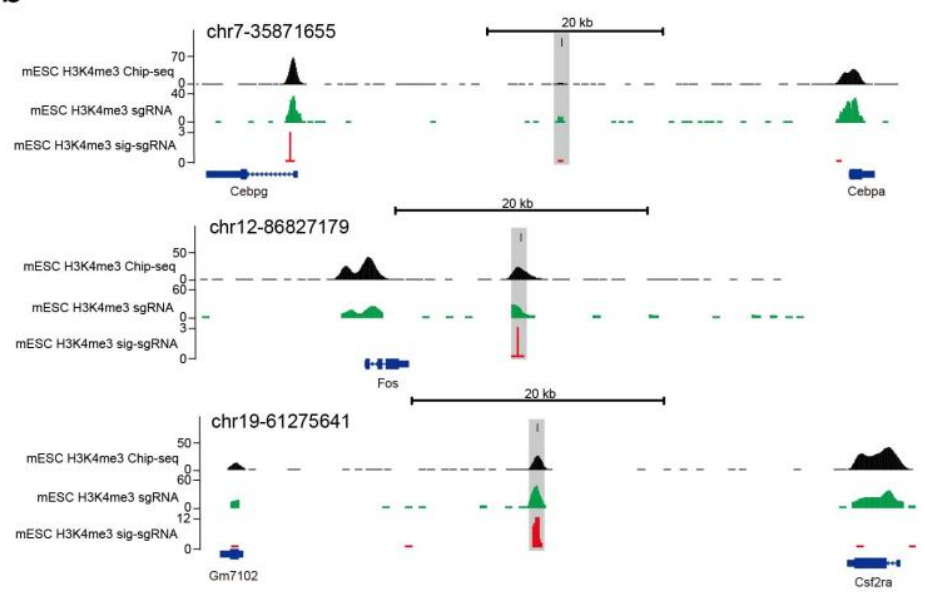

d
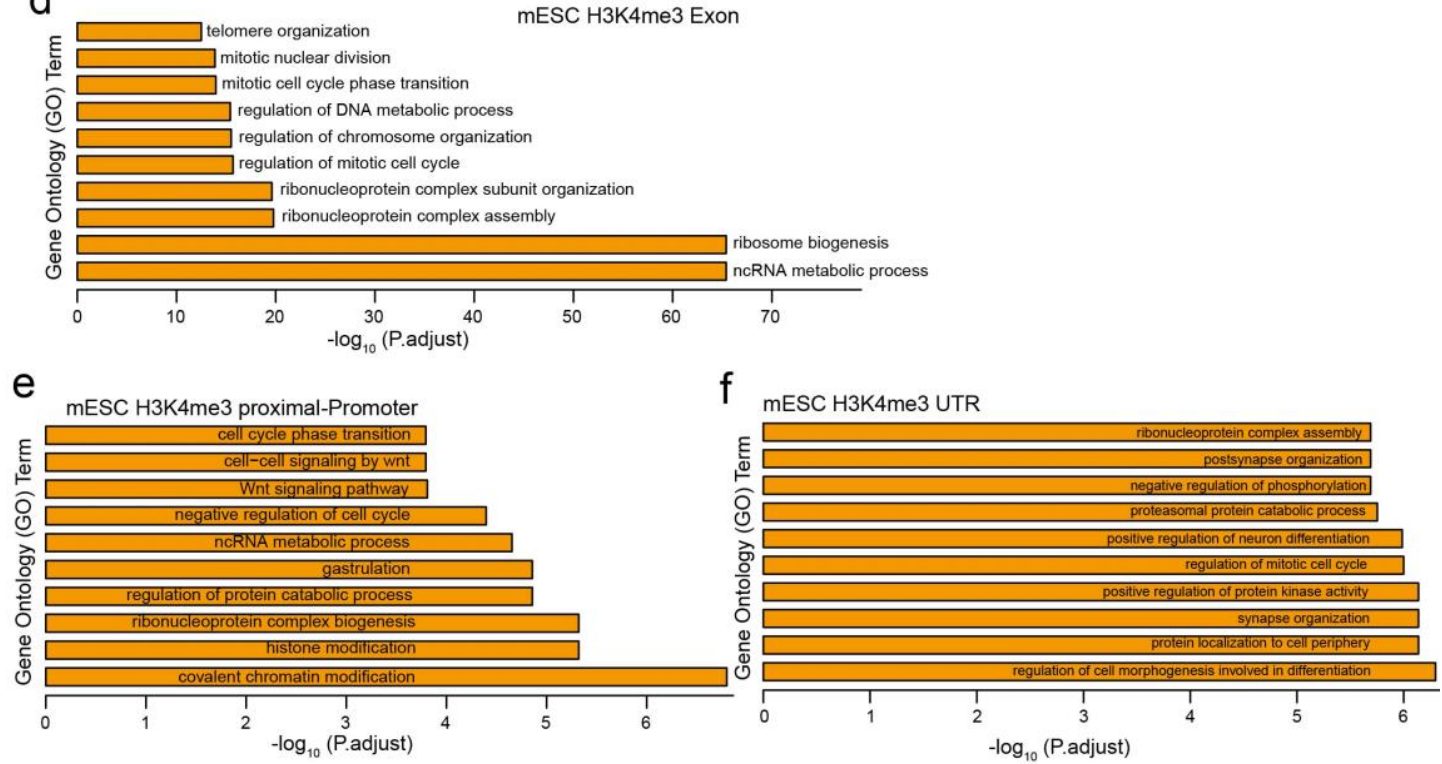

\section{$778 \quad$ Figure $\mathbf{S 3}$}

779 (a) Scatter-plot of the correlation between ChIP-seq reads number and sgRNA number in mESCs

780 H3K4me3 elements. Spearson's correlation coefficient with two-tailed test was calculated.

781 (b) Plots of H3K4me3 ChIP-seq reads, H3K4me3 sgRNAs and H3K4me3 ssgRNAs at three 782 genomic loci. Y-axes, RPKM. The genomic regions with H3K4me3 ssgRNAs validated in Figure 
Pan et al 2020

$7833 \mathrm{e}$ are shaded grey.

784 (c) Three H3K4me3 ssgRNAs validated in Figure 3e significantly inhibit mESCs proliferation. Cell 785 numbers of three time points are plotted. Error bars the SD of triplicates

786 (d) Top $10 \mathrm{GO}$ terms of the genes whose exons are targeted by H3K4me3 ssgRNAs.

787 (e) Top 10 GO terms of the genes whose proximal promoters are targeted by H3K4me3 ssgRNAs.

788 (f) Top $10 \mathrm{GO}$ terms of the genes whose UTRs are targeted by H3K4me3 ssgRNAs.

789

790

791

792 
Figure S4


C
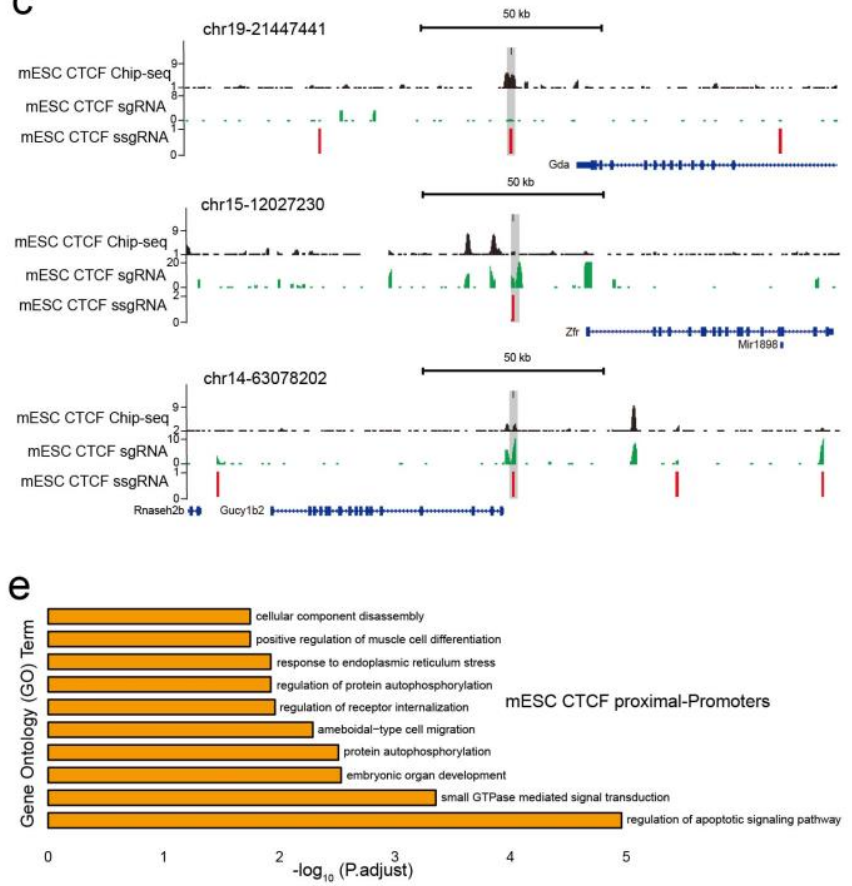

d
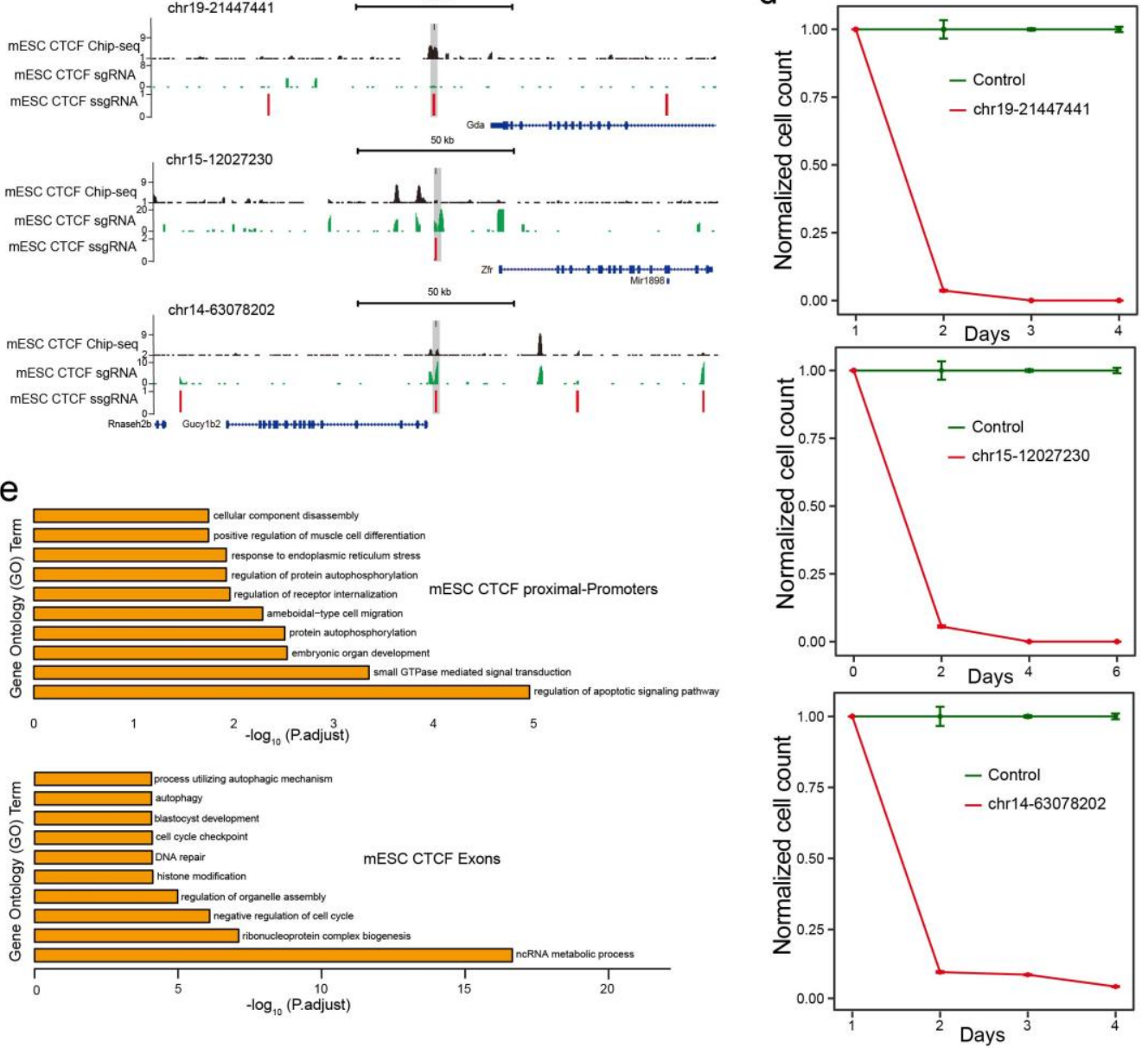

\section{Figure S4}

796 (a) Scatter-plot of the correlation between ChIP-seq reads number and sgRNA number in mESCs

797 CTCF elements. Spearson's correlation coefficient with two-tailed test was calculated.

798 (b) The distribution of CTCF ChIP-seq reads, CTCF sgRNAs and CTCF ssgRNAs on major 799 regulatory regions of mouse genome.

800 (c) Plots of CTCF ChIP-seq reads, CTCF sgRNAs and CTCF ssgRNAs at three genomic loci. Y801 axes, RPKM. The genomic regions with CTCF ssgRNAs (validated in Figure 4d) are shaded grey. 802 (d) Three CTCF ssgRNA (validated in Figure 4d) significantly inhibits mESCs proliferation. Cell 
Pan et al 2020

803

numbers of three time-points are plotted. Error bars the SD of triplicates.

804 (e) Top $10 \mathrm{GO}$ terms of the genes whose proximal promoters and exons are targeted by CTCF

805 ssgRNAs.

806

807

808 
Figure S5

a

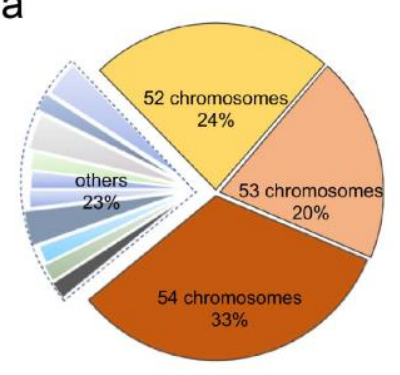

C

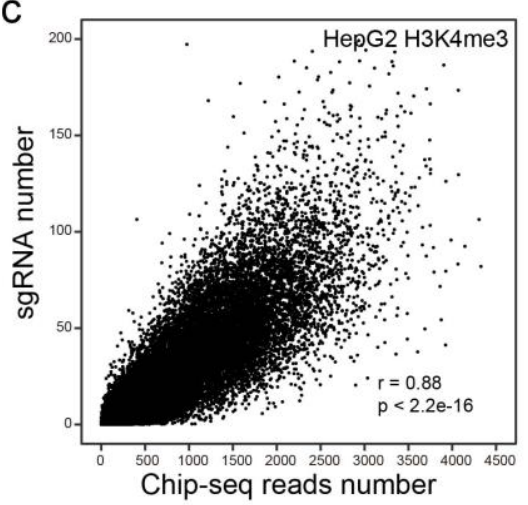

b HepG2 (ERR2355633)

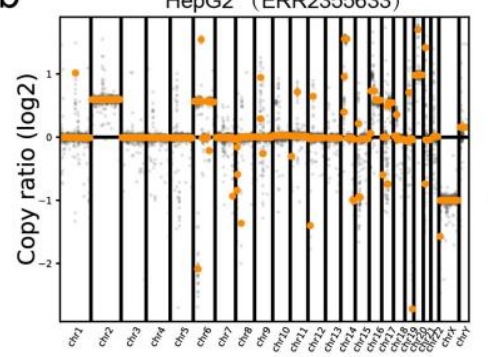

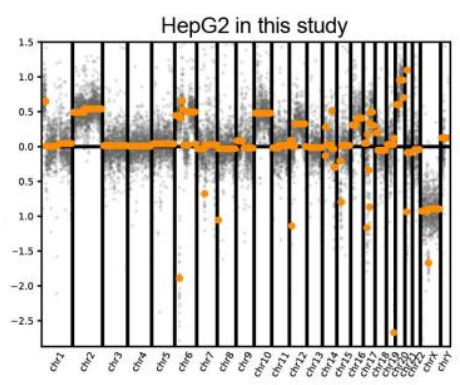

d

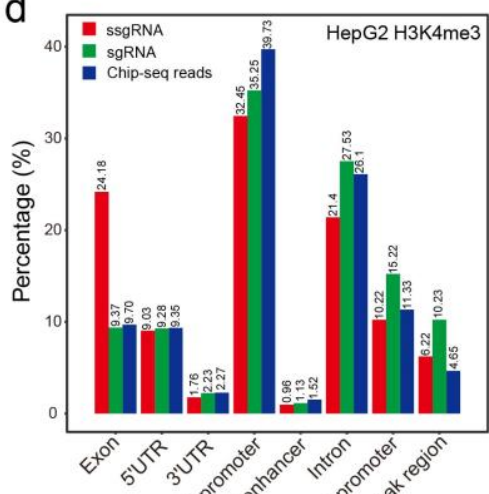

f

e

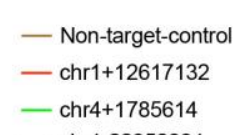

- chr1-22352881

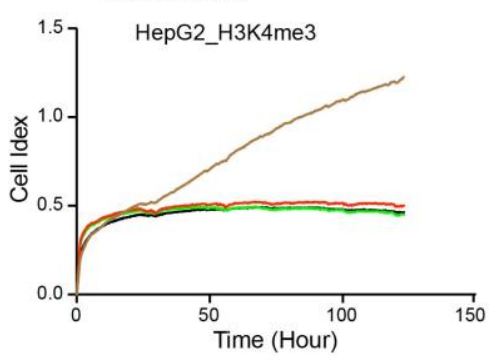

g
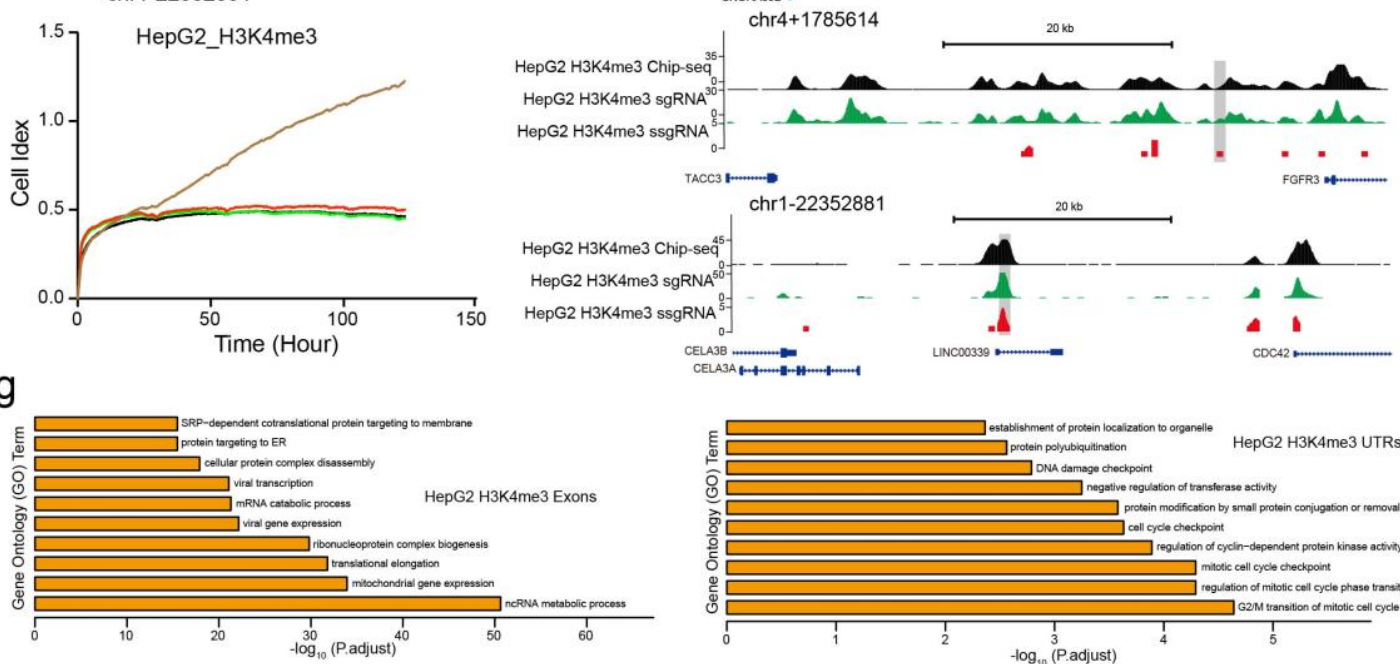

\section{Figure S5}

(a) Chromosome number of HepG2 used in this study based on 100 Giemsa-stained metaphases,

813 which illustrates that the modal number was 52-54 chromosomes.

(b) Copy Number Variation (CNV) analysis of HepG2 in this study through low depth whole genome sequencing. The CNV distribution of HepG2 used in this study is similar with that of a standard HepG2 cell from Sequence Red Archive (SRA) database (ERR2355633). H3K4me3 elements. Spearson's correlation coefficient with two-tailed test was calculated. 
Pan et al 2020

819 (d) The distribution of H3K4me3 ChIP-seq reads, H3K4me3 sgRNAs and H3K4me3 ssgRNAs on 820 major regulatory regions of human genome.

821 (e) All three H3K4me3 ssgRNAs (validated in Figure 5d) can significantly inhibit HepG2 822 proliferation. Real-time cell numbers are plotted.

823 (f) Plots of H3K4me3 ChIP-seq reads, H3K4me3 sgRNAs and H3K4me3 ssgRNAs at three 824 genomic loci. Y-axes, RPKM. The genomic regions with H3K4me3 ssgRNAs (validated in Figure $8255 \mathrm{~d})$ are shaded grey.

826 (g) Top $10 \mathrm{GO}$ terms of the genes whose exons and UTRs are targeted by H3K4me3 ssgRNAs.

827 
Figure S6

a

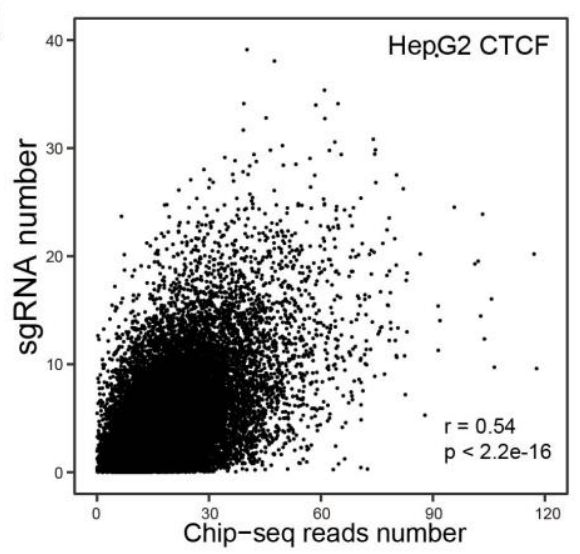

b

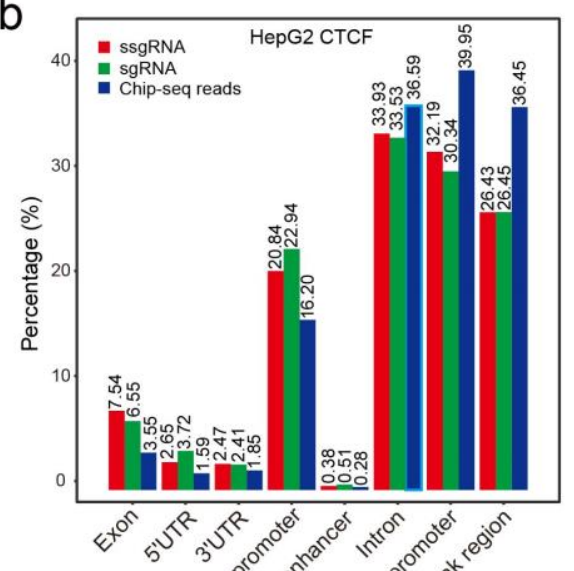

C

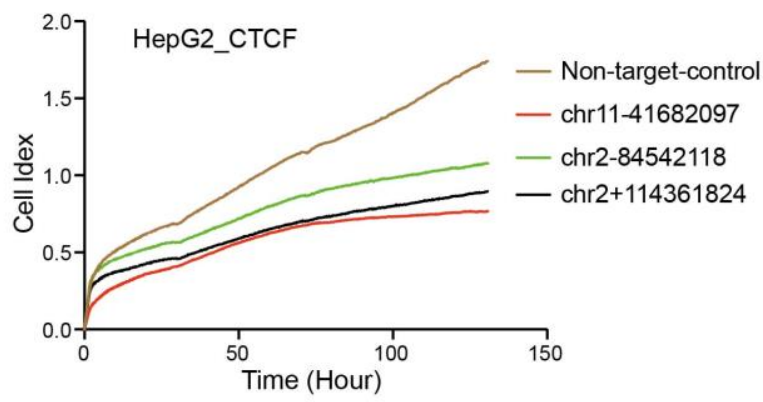

d
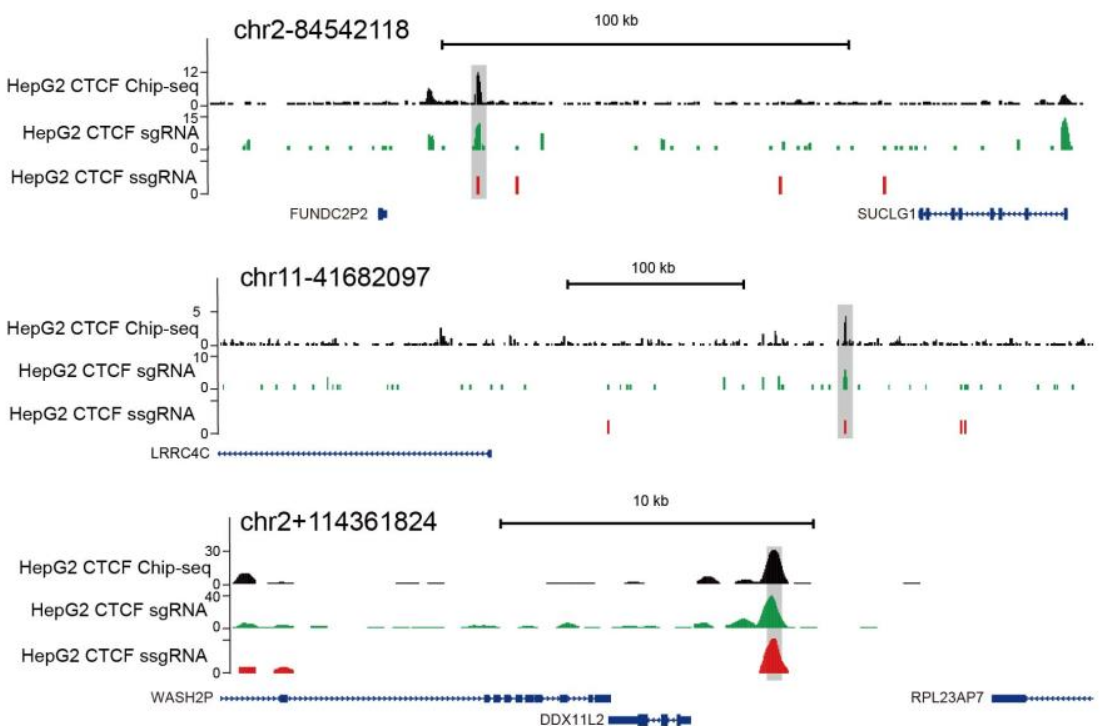

e
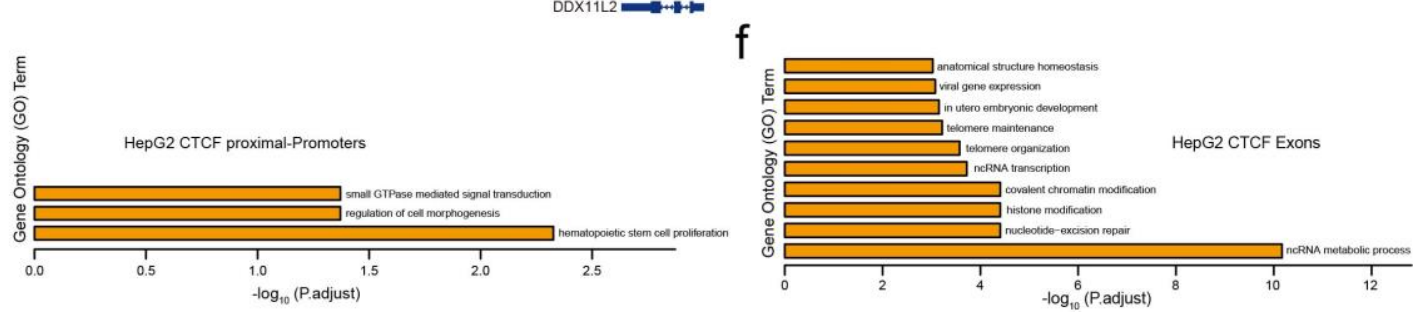

831 (a) Scatter-plot of the correlation between ChIP-seq reads number and sgRNA number in HepG2 832 CTCF elements. Spearson's correlation coefficient with two-tailed test was calculated.

833 (b) The distribution of CTCF ChIP-seq reads, CTCF sgRNAs and CTCF ssgRNAs on major 
Pan et al 2020

834 regulatory regions of human genome.

835 (c) All three CTCF ssgRNAs (validated in Figure 6d) can significantly inhibit HepG2 proliferation. 836 Real-time cell numbers are plotted.

837 (d) Plots of CTCF ChIP-seq reads, CTCF sgRNAs and CTCF ssgRNAs at three genomic loci. Y838 axes, RPKM. The genomic regions with CTCF ssgRNAs (validated in Figure 6d) are shaded grey.

839 (e) All three GO terms of the genes whose proximal promotors are targeted by CTCF ssgRNAs.

840 (f) Top 10 GO terms of the genes whose exons are targeted by CTCF ssgRNAs.

841 
Pan et al 2020

842

843

844

845

846

847

848

849

850

851

852

854

855

856 for 30min.

857

858

859

860 the library onto streptavidin beads.

861

862

We ligate the A3 adapter and amplify the library for ten cycles, and then digest the

863 library with BbsI (NEB, R3539). We fill in the gap with T4 DNA polymerase (NEB, 864

\section{Methods and Materials}

\section{Part 1: Wet lab experiments}

sgRNA library construction through CTDE (Controlled Template-dependent elongation)

\section{23bp DNA fragment generation}

Source DNA is fragmented. We ligate them to the A1 adapter and capture them onto T1 streptavidin magnetic beads (Thermo Fisher, 65601). Then we denature DNA with $0.1 \mathrm{M} \mathrm{NaOH}$ (Sigma, 79724) for 10min, and anneal primer for chain extension. We apply 2U Therminator ${ }^{\mathrm{TM}}$ DNA Polymerase (NEB, M0261) to incorporate one 3' hydroxyl-reversible dNTP (Jena Bioscience, 3'-O-N3-dNTPs). We restore the 3' hydroxyl group by $100 \mathrm{mM}$ TCEP (Sigma, 646547) treatment and repeat the incorporation-and-reversion for 22 cycles. We blunt DNA with mung bean nuclease (NEB, M0250) for 30min, and then apply T4 PNK (NEB, M0201) to phosphorylate it

\section{NGG PAM selection}

We ligate the A2 adapter and amplify the library for ten cycles. After the gel extraction of the amplified library, we digest the DNA with AscI (NEB, R0558). Then we capture

\section{NGG PAM removal}

M0203), ligate A4 adapter, and amplify the library with the KAPA HiFi polymerase 
865 mix (Roche, KK2631) for ten cycles. We apply 20\% TBE-PAGE to select the size of 866 the library (61nt). After releasing DNA from PAGE, we amplify the library by PCR 867 with KAPA HiFi polymerase and primer (the sequence is below) and size-selected via $8682 \%$ agarose gel ${ }^{1}$.

869 ArrayF

870 TAACTTGAAAGTATTTCGATTTCTTGGCTTTATATATCTTGTGGAAAGGAC

871 GAAACACCG

872 ArrayR

873 ACTTTTTCAAGTTGATAACGGACTAGCCTTATTTTAACTTGCTATTTCT

874 AGCTCTAAAAC

875 A1-F GAAAGGACGAAACACCgT

876 A1-R cGGTGTTTCGTCCTTTCCACaagatAGATCGGAAGAGCGTC-Biotin

877 Anneal Primer

$878 \operatorname{tgGACGCTCTTCCGATCTATCTTGTGGAAAGGACGAAACACCGT~}$

879 A2-F

880 CGCGCCCACACGTCTGAACTCCAGTCACAGTCAACAATCTCGTATGCCGT

881 CTTCTGCT

$882 \quad \mathrm{~A} 2-\mathrm{R}$

883 CAAGCAGAAGACGGCATACGAGATTGTTGACTGTGACTGGAGTTCAGACG

884 TGTGGGCGCG

885 A3-F 
A3-R

GCTTGAAGA

\section{Chromatin Immunoprecipitation (ChIP)}

894 The ChIP assay was performed according to manufacture of Millipore ChIP kit 895 (Millipore, 20-153). Briefly, formalin was added to cells to crosslink protein and DNA 896 and then cells $\left(10^{6}-10^{7}\right)$ were lysed in SDS lysis buffer. Chromatin was fragmented via 897 sonication to the size of 200bp-500bp and then immunoprecipitated with antibodies (5$89815 \mu \mathrm{g}$ each sample). The enriched DNA was purified by QIAquick PCR purification kit 899 (QIAGEN). These DNA samples were applied to CTDE protocol or illumina 900 sequencing library construction. The antibodies are anti-H3K4me3 (Cell Signaling, 901 9751) and anti-CTCF (Millipore, 07-729).

902 903

\section{Cell culturing}

904 V6.5 ESCs were maintained in DMEM supplemented with $15 \%$ fetal bovine serum 905 (Gibco), 0.1mM $\beta$-mercaptoethanol, 2mM L-glutamine, $0.1 \mathrm{mM}$ nonessential amino 906 acid, 1000U/ml recombinant leukemia inhibitory factor (Millipore LIF, ESG1107), and 
Pan et al 2020

907

908

909

910

911

912

913

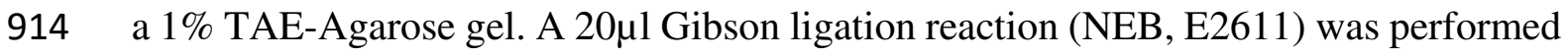
915

916

917

918

919

920

921

922

923

924

925 Lentivirus production and transduction

926 Lentivirus was produced through the co-transfection of the lentiviral vectors with

927 psPAX2 (Addgene, \#12260) and pMD2.G (Addgene, \#12259) into HEK293T cells 
928

929 930 931 932 933 934 935 936 937 applied these cells for dropout screen.

938

\section{CRISPR/Cas9 based dropout screen}

940 After puromycin selection, 4 million cells were seeded into a 10-cm dish every 941 generation. 10 million cells from the 1st generation and 10th generation were used for 942 genomic DNA extraction and sgRNA-template targeted sequencing. For DNA 943 extraction, we used ethanol-precipitated method to avoid DNA loss. Cell pellet was

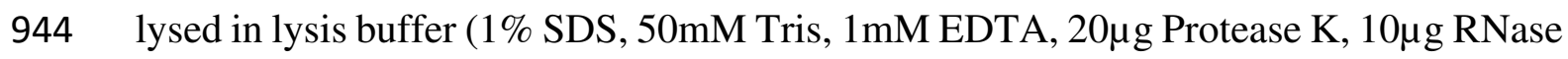
945 A) in $65^{\circ} \mathrm{C}$ water bath overnight. Cell lysate was denatured by 946 phenol:chloroform:isopentanol (25:24:1), and thoroughly mixed by shaking. After 947 centrifugation, DNA was precipitated from supernatant by 2 volume $100 \%$ ethanol. We 
washed DNA pellet twice with chilled $70 \%$ ethanol. After air drying, we dissolved

949 DNA in $\mathrm{ddH}_{2} \mathrm{O}$.

950

\section{1 sgRNA-template targeted sequencing}

952 Two PCR steps were performed:

953 1. PCR from enough genomic DNA to preserve library complexity.

954 sgRNA template containing cassette was amplified using primers specific to the 955 lentiCRISPR v2 vector (Round1-F and Round1-R)

956 Round1-F AATGGACTATCATATGCTTACCGTAACTTGAAAGTATTTCG

957 Round1-R CCAACTTCTCGGGGACTGTGGGCGATGTGCGCTCTGCCCACTGA

$95824 \times 50 \mu \mathrm{l}$ PCR reactions were performed with $2 \mu \mathrm{g}$ genomic DNA in each tube to

959 achieve over $100 \times$ coverage. 20 cycles were used for minus bias.

960 2. Add illumina sequencing adapters.

$96110 \mu 1$ DNA from first round PCR product (combined all tubes together) was used as

962 template for the second round PCR. Second round PCR product was purified by

963 QIAquick PCR clean-up Kit and quantified by Agilent 2200 Bio-analyzer before

964 illumina sequencing.

965 Round 2-F

966 AATGATACGGCGACCACCGAGATCTACACTCTTTCCCTACACGACGCTCTT

967 CCGATCTtcttgtggaaaggacgaaacaccg

968 Round2-R 


\section{Anti-neomycin cell-line preparation}

973 Anti-Neomycin mESCs cells were performed as described with modifications ${ }^{3}$. Briefly, 974 the pEF1a-BirA-V5-neo (\#100548, Addgene) plasmid was stably transfected into V6.5

975 ESCs via electroporation. Cells were selected by neomycin and were applied to screen 976 experiment.

978 Commercial synthesis Neo sreen library contruction

979 According to 795bp neo fragment sequence, we pick all gRNA with NGG PAM, totally

980115 gRNA. Also, we chose 20 gRNA from Gecko negative control (they can't target

981 hg19, mm9 and neo reference sequence). We order these gRNA from oligo synthesis 982 company (Shangya), and these oligoes were in uniform format: 5'-

983 GTGGAAAGGACGAAACACCGNNNNNNNNNNNNNNNNNNNNGTTTTAGA

984 GCTAGAAATAGC-3', where N20 represent neo gRNA or negative control sequence.

985 We combined 115 neo gRNA and 20 negative control equally together, and amplified $9865 \mu$ library $(10 \mu \mathrm{M})$ with $2 \mathrm{X}$ HiFi DNA polymerase, Array-F and Array-R primer for 5 987 cycles. 140bp library was gel extracted and assembled into LentiCRISPR V2 plasmid. 98820 negative control were separately prepared as above and assembled into 989 LentiCRISPR v2 plasmid, and this library was spike-in of CTDE-neo library. 


\section{$991 \quad$ Neo fragment obtaining}

992 We obtained neo fragment directly by PCR using pEF1a-BirA-V5-neo (Addgene, 993 \#100548) as template, and gel extract 795bp DNA as source of CTDE method to 994 construct CTDE neo library.

\section{Neo library Screen experiment}

997 After puromycin selection, 1 million cells were seeded into a 10-cm dish, and 1 million 998 cells were collected and labelled with Prev-selection. Anti-Neomycin mESCs infected by CTDE-Neo and Commercial synthesis Neo library were cultured with $150 \mu \mathrm{g} / \mathrm{ml}$

1000 Neomycin (Sangon) for 7 days. 7 days later, cells were collected and labelled with 1001 Post-selection. Prev- and Post-selection sample were extracted genomic DNA and 1002 illumina sequencing library were construct as above.

1004 Plasmid construction

1005 Oligoes (for sgRNA or shRNA) were annealed by gradient cooling process and were 1006 ligated to lentiCRISPR v2 (BsmBI digested) or PLKO.1 (digested by AgeI/EcoRI) 1007 using T4 DNA ligase. After transformation, colonies were picked. The confirmed 1008 colonies by sanger sequencing were preserved in glycerol and plasmid were applied to 1009 transfection. The CDC42 cDNA was obtained from ORF collection (Ultimate ${ }^{\mathrm{TM}}$ ORFs, 
Pan et al 2020

1010

GeneID: 998). The entire open-reading frame was amplified by PCR and cloned into

1011 PLVX vector at BamHI and XhoI sites.

1012

1013 AP staining and survival assay

1014 mESCs transduced with lenti-sgRNA were first selected under $1 \mu \mathrm{g} / \mathrm{ml}$ puromycin for 10153 days. AP staining was performed according to the manufacturer's instructions 1016 (Beyotime Biotechnology, C3206). HepG2 cells transduced with sgRNA or 1017 LINC00339-shRNA were plated 5 days after transduction at $1.8 \times 10^{5}$ cells per well in 1018 a 24-well plate. Then cells were plated in 6-well plates at a density of 3000 cells per 1019 well. The culture media with $1 \mu \mathrm{g} / \mathrm{ml}$ puromycin was refreshed every 3 days for $\sim 14$ 1020 days. After PBS washing, colonies were stained with $0.1 \%$ crystal violet (Beyotime,

1021 C0121) for 10min at room temperature. Then the images of each well were recorded by 1022 a digital camera and the number of colonies was counted using ImageJ software.

1023

1024 Karyotype analysis

1025 HepG2 $\left(10^{5}\right)$ were trypsinized and resuspended in $5 \mathrm{ml} 0.56 \% \mathrm{KCl}$ in water. After 1026 incubation at $37^{\circ} \mathrm{C}$ for $10 \mathrm{~min}, 1 \mathrm{ml} 25 \%$ freshly made fixative solution (methanol:glacial 1027 acetic acid $=3: 1$ by volume) in water was added. Cells were pelleted and resuspended 1028 in $1 \mathrm{ml} 100 \%$ fixative solution three times. To make cells spread, one drop of the cell 1029 suspension was dropped (from a height of $0.5 \mathrm{~m}$ ) onto a glass slide and allowed to air 
Pan et al 2020

1030 dry before DAPI staining. The mitotic chromosome number was counted under the

1031 fluorescence microscopy.

1032

\section{Cell cycle analysis}

1034 Cells were transduced with lentivirus via spinfection in 6-well plates. After puromycin 1035 selection, cells were harvested and fixed in chilled $70 \%$ ethanol at $-20^{\circ} \mathrm{C}$ overnight, and 1036 stained with $40 \mu \mathrm{g} / \mathrm{ml}$ propidium iodide (Sigma-Aldrich, P4170) containing $50 \mu \mathrm{g} / \mathrm{ml}$ 1037 RNase (Sigma-Aldrich, R6513) at $37^{\circ} \mathrm{C}$ for $15 \mathrm{~min}$ in the dark, then analyzed cell cycle 1038 by FACSCalibur flow cytometer (BD Biosciences). Data were analyzed with ModFit 1039 LT software.

1040

1041 qPCR quantification

1042 Total RNA was harvested using the RNeasy Plus Mini Kit (Qiagen, 74134) and $1 \mu \mathrm{g}$

1043 RNA was used for reverse transcription with the PrimeScript ${ }^{\mathrm{TM}}$ RT Master Mix (Takara, 1044 RR036A). After reverse transcription, TB Green® Fast qPCR (Takara, RR430A) was 1045 performed with related primers (Table S14).

1046

\section{Western blot}

1048 Cells were harvested and lysed in SDS-PAGE sample buffer. Equal amounts of total 1049 protein were loaded in each lane. Proteins were resolved by SDS-PAGE, transferred to $1050 \quad 0.45 \mu \mathrm{m}$ PVDF membranes, and probed with the indicated antibodies. The antibodies 
Pan et al 2020

1051

1052

1053

1054

1055

1056

1057

1058

1059

1060

1061

1062

1063

1064

1065

1066

1067

1068

1069

1070

used for western blotting are: anti-CDC42 (Cell Signaling Technologies, 2466), anti- $\beta$ tubulin (Cell Signaling Technologies, 2128).

\section{Real-Time cell number analysis.}

Experiments were performed using Real-Time Cell Analyzer (iCELLigence Analyzer, ACEA Biosciences Inc.) at $37^{\circ} \mathrm{C}$ with $5 \% \mathrm{CO} 2$. To measure the background, we placed 200 $\mu 1$ DMEM medium in the E-plate L8 (ACEA Biosciences, 00300600840). Then, we added $400 \mu \mathrm{l}$ HepG2 (transduced with sgRNA or shRNA) cell suspension (8000 cells/well) with 10\% FBS DMEM medium. The impedance (cell index), which was detected by sensor electrodes in E-plate L8 every $15 \mathrm{~min}$ for 120 hours. The plot of the cell index was calculated automatically by the RTCA software package 1.1.1 (ACEA, Biosciences Inc.).

\section{Part 2: Dry lab experiments and data analysis}

\section{Sequencing data pre-processing}

The designed lentiCRISPR v2 sgRNA fragments were sequenced. The sequenced read comprises a 5'-adapter, a sgRNA, and a 3'-adapter, one by one. We trimmed the read, remove the pre-designed 5'-adapter and 3'-adapter (Table S14) to get the sequence of the sgRNA. Then we adhered "NGG" to the end of each sgRNA to recover its PAM sequence and prepared it for mapping on the reference genome. 
Pan et al 2020

1072

The assessment of sgRNA library generated by CTDE from plasmid lentiCRISPR

1073

v2

1074 1. We evaluated the detected sgRNAs from the designed reference of plasmid 1075 lentiCRISPR v2 (Addgene \#52961, 14873bp) with the following steps:

1076 Step 1, we built a mapping reference set from the plasmid lentiCRISPR v2 sequences 1077 using Bowtie build-index function.

1078 Step 2, we prepared three sgRNA libraries with the CTDE approach. For each of the 1079 libraries, we sampled one million reads and then extracted 19-21 bp length sgRNAs 1080 from the "Sequencing data pre-processing" analysis. We merged three sgRNA libraries 1081 to get a sgRNA set.

1082 Step 3, we aligned the sgRNAs set from step2 to the reference set by the bowtie aligner,

1083 allowing one base mismatch and reporting all alignments (-v 1 -a).

1084 Step 4, we counted the number of mapped sgRNAs as x. We identified the "NGG" 1085 locus on either sense or antisense strands of the plasmid lentiCRISPR v2 sequences.

1086 For locus i, we counted the number of sgRNAs whose PAM tails mapped to the locus 1087 as $y$. Then we calculated the ratio of $\operatorname{sgRNAs}$ for locus i (Ratio locus $\__{i}$ ) as follows:

$$
\operatorname{Ratio}_{\text {locus } i} i=\frac{y}{x} \times 100
$$

1089 We repeatedly calculated the number and ratio of sgRNAs for each locus. Next we 1090 divided lentiCRISPR v2 sequences into equal-sized $10 \mathrm{bp}$ bins and calculated the ratio 1091 of AT for each bin. We finally used Integrative Genomics Viewer (IGV) (v2.8.0) to 
1092

1093

1094

1095

1096

1097

1098

1099

1100

1101

1102

1103

1104

1105

1106

1107

1108

1109 Step 2, we sampled one million reads from each of three sgRNA libraries, then we got

1110 the sgRNAs from the "Sequencing data pre-processing" analysis and extracted the

1111 sgRNAs of 20bp length. 
1112 Step 3, we processed the sgRNAs in two different ways. In one way, we removed their

1113 "NGG" tails and mapped them to the reference set by bowtie, not allowing base

1114 mismatch (-v 0). Simultaneously, we mapped them to the reference set by bowtie,

1115 allowing one base mismatch (-v 1). After alignment, we counted the number of mapped

1116 sgRNAs as $N_{N G G-P A M}$. Then the fold enrichment of the detected sgRNAs on the "NGG"

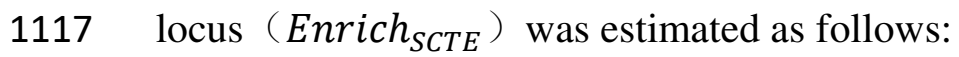

$$
\text { Enrich }_{S C T E}=\frac{N_{N G G-P A M}}{N_{\text {total }} \times P_{N G G-P A M}}
$$

1119 4. We grouped the detected sgRNAs by their length and calculated the proportion of sgRNAs of different length with the following steps:

1121 Step 1, for each of the three sgRNA libraries, we got sgRNAs from the "Sequencing

1122 data pre-processing" analysis and mapped them to reference set by bowtie, allowing

1123 one base mismatch (-v 1).

1124 Step 2, we grouped sgRNAs by their length. For the sgRNAs of length i, we counted

1125 the number as $N_{i}$, and calculated their proportion as follows:

$$
\text { Proportion }_{i}=\frac{N_{i}}{N_{\text {all }}}
$$

1127 Here $N_{\text {all }}$ is the number of all mapped sgRNAs. We repeatedly calculated the 1128 proportion for the sgRNAs of 16-24bp from the three sgRNA libraries.

1129 5. To evaluate the how faithful sgRNA synthesis was, we mapped 16-24bp sgRNA to 1130 reference set by the following priority order: with no mismatch, with one mismatch, 1131 with two mismatches (not include mismatch in PAM site). For PAM site, we only 1132 tolerate mismatch at the first base. The error rate was calculated as total number of 
Pan et al 2020

1133

1134 site).
1136

1137

1138

1139

1140

1141

1142

1143 reads count of control sgRNA in pre- and post-selection library. After normalization,

1144 the fold change of each sgRNA between post- and pre-selection library was calculated.

1145 Targeting sgRNAs whose fold change less than the minmum fold change of non-

1146 targeting control sgRNAs were recognized as ssgRNA.

\section{Comparison between NSgRNAShot and MAGeCK in identify ssgRNA}

1149 We downloaded one genome-wide CRISPR-Cas9 dropout screen data of mESCs as the

1150 benchmark data ${ }^{4}$. MAGeCK is a widely used method to identify of essential genes from

1151 genome-scale CRISPR/Cas9 knockout screens ${ }^{5}$. As described in the previous paper,

1152 since the CRISPR/Cas9 knockout system should show no difference in selection

1153 preference between control samples or between replicated treatment samples, a good 
Pan et al 2020

1154 method should not detect many significantly selected sgRNAs and genes between these

1155 samples $^{5}$. So we took the strategy to detect the possible false-positive ssgRNA with

1156 FDR $<0.1$ between two replicates of ESC treatment samples with NSgRNAShot and

1157 MAGeCK based on benchmark data. When multiple sgRNAs are available for one gene,

1158 MAGeCK demonstrated the best performance to detect essential genes ${ }^{6}$. Therefore we

1159 evaluated the sensitivity of ssgRNA identified by our method with the following

1160 strategy: we took the essential genes reported by MAGeCK as the gold standard set, a

1161 good method should largely report the ssgRNA from essential genes but not from other

1162 genes.

1163

1164 ChIP-seq data analysis

1165 For ChIP-seq of H3K4me3 and CTCF in mESC and HepG2 cell lines, libraries were

1166 sequenced using Illumina HiSeq X Ten and paired-end 150 bp long reads were obtained.

1167 Chip-seq reads were analyzed with the following steps:

1168 Step 1, we trimmed Chip-seq reads to $100 \mathrm{bp}$ (from 5' to 3'), and for redundant reads

1169 which have the same sequence, only one was retained. Then reads were mapped to the

1170 reference genome (mm9 for $\mathrm{mESC}$ or hg19 for HepG2) by bowtie, only uniquely

1171 mapped reads were reported (-m 1).

1172 Step 2, Chip-seq peaks for H3K4me3 and CTCF were detected by MACS2 with default

1173 settings. For each mouse ES CTCF peak, we calculated its length (peak length) and 
Pan et al 2020

1174 counted the number of reads mapped on the peak (number of mapped reads), then

1175 we calculated its average read depth (average depth per peak) as follows:

1176

$$
\text { average depth per peak }=\frac{\text { reads length } \times \text { number of mapped reads }}{\text { peak length }}
$$

1177 We filtered peaks whose average read depth less than ten.

1178 Step 3, we manually identified regions which consistently contain significantly

1179 enriched reads in multiple input ChIP-Seq data provided by Encode project (Table S15)

1180 and regarded them as false positive peak regions. We filtered the peaks overlapped with

1181 these regions.

1182

\section{$1183 \quad$ RNA-seq data analysis}

1184 1. We downloaded two total RNA-seq datasets of HepG2 from Gene Expression

1185 Omnibus (GEO) database (GEO:GSE88089), and two total RNA-Seq datasets of

1186 mESC from Beijing Institute of Genomics (BIG) Data Center (CRA001133).

1187 2. For each RNA-seq dataset, raw reads were mapped to reference genome ( $\mathrm{mm} 9$ for

$1188 \mathrm{mESC}$ or hg19 for HepG2) by bowtie, allowing one base mismatch and only uniquely

1189 mapped reads were retained (-v $1-\mathrm{m}$ 1). For redundant reads that fall on the same

1190 position on the genome, only one was retained.

1191 3. Based on the UCSC knownGene annotations, we counted mapped reads in the exon

1192 regions of transcripts, and then we calculated transcript expression levels by

1193 normalizing the number of reads for each transcript to total mapped reads and mRNA

1194 length, namely reads per kilo-bases per million reads (RPKM). 
Pan et al 2020

1195 4. We got the average RPKMs of transcripts for mESC and HepG2, respectively, and 1196 the largest RPKM of transcripts for each gene represents gene expression level. We 1197 selected the genes with RPKM higher than one as expressed genes.

1198

\section{Whole-genome sequencing data analysis}

1200 SNP (single nucleotide polymorphism) existed in the human and mouse population. To

1201 identified the sgRNAs from genomic regions with SNP, we built additional reference 1202 from the regions with the following steps:

1203 1. We downloaded one whole-genome sequencing (WGS) dataset of HepG2 from the

1204 Sequence Read Archive (SRA) database (ERR2355633). Moreover, the WGS library

1205 of mESC was sequenced using Illumina HiSeq X Ten, and paired-end $150 \mathrm{bp}$ long reads

1206 were obtained.

1207 2. For each WGS dataset, paired-end reads were merged together. Then we trimmed

1208 adapter residues from reads by AdapterRemoval (Version 2.1.7), and reads shorter than

$1209150 \mathrm{bp}$ after trimming were filtered out.

12103 . We performed step-wise mapping process, step 1, reads were mapped to the reference

1211 genome (mm9 for mESC and hg19 for Hepg2) using bowtie2 with default settings. Step

12122 , mapped reads from step 1 were mapped to the reference genome using bowtie, not

1213 allowing base mismatch (-v 0). Unmapped reads from step 2 were regarded as derived

1214 from regions of mESC or HepG2 with SNP, we used these uniquely unmapped reads

1215 to build a mapping reference set using the Bowtie build-index function. 


\section{Identification of negatively selected sgRNAs}

1218 1. The sgRNA libraries generated by H3K4me3 or CTCF labeled DNA sequences were 1219 sequenced using Illumina HiSeq X Ten. For each sgRNA library, we got sgRNAs from 1220 "Sequencing data pre-processing” analysis.

1221 2. We selected 18-21 bp length sgRNAs which have ability to direct Cas9-induced 1222 indels at target sites for further analysis. Then we performed step-wise mapping process. 1223 Step 1, sgRNAs were mapped to reference genome (mm9 for mESC and hg19 for 1224 HepG2) using bowtie, allowing one base mismatch (-v 1). Step 2, unmapped sgRNAs 1225 from step 1 were mapped to reference set from "Whole-genome sequencing data 1226 analysis" using bowtie, allowing one base mismatch (-v 1). We counted the number of 1227 each mapped sgRNA from above two steps.

1228 3. The sgRNAs whose abundance had significantly reduced from P1 to P10 were 1229 identified using our designed NSgRNAShot algorithm. We used the term 'ssgRNA' to 1230 refer to negatively selected sgRNA.

1232 Genome-wide distribution of chip-seq reads, sgRNAs and ssgRNAs

1233 We analyzed the genomic distribution of chip-seq reads, sgRNAs, and ssgRNAs with 1234 following steps: 
Pan et al 2020

1235 1. We got sgRNAs from "Identification of negatively selected sgRNAs". Then we 1236 mapped sgRNAs to reference genome ( $\mathrm{mm} 9$ for mESC and hg19 for HepG2) using

1237 bowtie, allowing one base mismatch (-v 1$)$.

1238 2. We got ssgRNAs from "Identification of negatively selected sgRNAs" and mapped

1239 them to reference genome using bowtie, allowing one base mismatch and refraining

1240 from reporting any alignments have more than three reportable alignments (-v $1-\mathrm{a}-\mathrm{m}$

1241 3). For ssgRNAs having more than one reportable alignment, we only retained

1242 alignments located in peak region, otherwise, all alignments were retained.

1243 3. We calculated the chip-seq reads, sgRNAs, and ssgRNAs density on each $100 \mathrm{bp}$

1244 window and sliding along the chromosomes with a step length of $20 \mathrm{bp}$. We used Circos

1245 (v0.69-6) tool to display the genome-wide density distribution of chip-seq reads,

1246 sgRNAs and ssgRNAs, and local density distribution was visualized by the UCSC

1247 Genome Browser.

1248 4. To display the distribution of chip-seq reads, sgRNAs and ssgRNAs in functional

1249 elements of the genome, we assigned annotation for each nucleotide in the reference

1250 genome by using the following priority order: coding-exon $>5$ '-UTR $>3$ '-UTR $>$

1251 proximal promoter $(10 \mathrm{~kb}$ upstream of a TSS $)>$ super-enhancer $>$ intron $>$ distal

1252 promoter $(>10 \mathrm{~kb}$ upstream of a TSS $)=$ non-peak region. We assigned chip-seq read,

1253 sgRNAs and ssgRNAs to these categories based on location.

1254 5. To demonstrate what the distribution of chip-seq reads, sgRNAs and ssgRNAs per 1255 peak look like, we split each peak into non-overlapping 30 bins. Then for each of these 
Pan et al 2020

1256

1257 for visualization separately. Peaks were ranked from highest to lowest based on median

1258 RPKM of chip-seq reads of bins.

\section{Recall rate of essential genes from CTDE}

1261 We downloaded two gene sets described as essential for survival and proliferation of $1262 \mathrm{mESCs}^{4,7}$. The overlap of two gene sets was used as reference essential genes for 1263 mESCs. We mapped sgRNAs and ssgRNAs to reference genome (mm9) using bowtie 1264 (-v 1 -m 1), essential genes whose exons were targeted by more than 2 sgRNA were 1265 regarded as covered by CTDE. The ratio of essential genes, which are covered by

1266 CTDE, targeted by ssgRNA is the recall rate of essential genes.

\section{Gene Ontology (GO) analysis}

1269 We assessed whether related genes of exon, intron, utr, and proximal promoters covered

1270 by ssRNAs were enriched for particular GO categories by calculating adjusted P-value

1271 using the Benjamini \& Hochberg method. We performed GO analyses using R package

1272 clusterProfiler with default parameters.

1273 1. For uniquely mapped ssgRNAs generated by H3K4me3 labeled DNA sequences, 1274 only related expressed genes (FPKM > 1) of an exon, utr, intron, and proximal 1275 promoters with ssgRNAs located were used to perform GO analysis. 
Pan et al 2020

1276 2. For uniquely mapped ssgRNAs generated by CTCF labeled DNA sequences, all

1277 related genes of utr, intron and proximal promoters with ssgRNAs located were used

1278 for GO analysis, but only related expressed genes of exons with ssgRNAs located were

1279 used for GO analysis.

1280 3. We sorted all GO categories according to adjusted P-values in an ascending order.

\section{Analysis of CTCF hallmarks shared occupation in different cell types}

1. We downloaded CTCF hallmarks of 16 mouse cell lines and tissues from ENCODE, the CTCF hallmarks of mESC were occupied in higher than 9 of 16 mouse cell lines and tissues were defined as common hallmarks.

2. We downloaded CTCF hallmarks of 55 human cell lines from ENCODE, the CTCF

1287 hallmarks of HepG2 were occupied in higher than 33 of 55 human cell lines were 1288 defined as common hallmarks.

1289 3. We mapped ssgRNAs from "Identification of negatively selected sgRNAs" to 1290 reference genome (mm9 for mESC and hg19 for HepG2) using bowtie, allowing one 1291 base mismatch and reported uniquely mapped reads (-v $1-\mathrm{m} 1)$. Based on the position 1292 of CTCF hallmarks and ssgRNAs in the genome, we calculated the distribution of 1293 ssgRNAs in common and cell-type specific CTCF hallmarks. We used the term 1294 'essential common peaks' to refer to common CTCF hallmarks with ssgRNAs located. 
1298 We assumed that in the dish, during culture, 1) most sgRNAs do not change their 1299 abundance, which suggests they suffer no selection pressure; 2) no sgRNAs is 1300 undergoing positive selection and increases the abundance; 3) a few sgRNAs are 1301 suffering negative selection and decrease their abundance. Then we developed a 1302 method named Negative-SgRNA-Shot (NSgRNAShot) to detect significant1303 negatively-selected sgRNAs (ssgRNA). Here are the details.

1304 Step 1. We got reads counts (r) profiles of sgRNAs at 1st generation (p1) and 10th 1305 generation (p10) in the dish from "Identification of negatively selected sgRNAs" 1306 analysis. Suppose we have detected $\mathrm{n}$ and $\mathrm{m}$ sgRNAs from $\mathrm{p} 1$ and $\mathrm{p} 10$, then we 1307 calculated the sequencing depth of $\operatorname{sgRNAs}$ profiles at $\mathrm{p} 1\left(\mathrm{Sum}_{p 1}\right)$ and $\mathrm{p} 10\left(\mathrm{Sum}_{p 10}\right)$ 1308 with the following formula,

$$
\operatorname{Sum}_{p 1}=\sum_{i=1}^{n} r_{i}, \operatorname{Sum}_{p 10}=\sum_{j=1}^{m} r_{j}
$$

1310 If $\operatorname{Sum}_{p 1} \geq \operatorname{Sum}_{p 10}$, we calculated the normalized reads counts ( $\mathrm{nr}$ ) of sgRNAs at $\mathrm{p} 1$

1311 with the formula: $\mathrm{nr}_{i}=\mathrm{r}_{i}$, where $1 \leq \mathrm{i} \leq \mathrm{n}$; and we calculated the normalized reads 1312 counts of $\operatorname{sgRNAs}$ at p10 with the formula: $\mathrm{nr}_{j}=\mathrm{r}_{j} *\left(\operatorname{Sum}_{p 1} / \operatorname{Sum}_{p 10}\right)$, where $1 \leq$ $1313 \mathrm{j} \leq \mathrm{m}$.

1314 If $\operatorname{Sum}_{p 1}<\operatorname{Sum}_{p 10}$, we calculated the normalized reads counts of sgRNAs at $\mathrm{p} 1$ with 1315 the formula: $\mathrm{nr}_{i}=\mathrm{r}_{i} *\left(\operatorname{Sum}_{p 10} / \operatorname{Sum}_{p 1}\right)$, where $1 \leq \mathrm{j} \leq \mathrm{n}$; and we calculated the 1316 normalized reads counts of $\operatorname{sgRNAs}$ at p10 with the following formula: $\mathrm{nr}_{j}=\mathrm{r}_{j}$, 1317 where $1 \leq \mathrm{j} \leq \mathrm{m}$. 
1318 Step 2. We calculated $\log 2$ fold change $(\log 2 \mathrm{fc})$ value of abundance of a sgRNA from

$1319 \mathrm{p} 1$ and p10 with the following formula:

$$
\log 2 \mathrm{fc}=\log 2\left(\frac{\max \left\{\mathrm{nr}_{p 1}, 10\right\}}{\max \left\{\mathrm{nr}_{p 10}, 10\right\}}\right)
$$

1321 where $\mathrm{nr}_{p 1}$ and $\mathrm{nr}_{p 10}$ are the normalized reads count of the sgRNA at $\mathrm{p} 1$ and $\mathrm{p} 10$.

1322 However, if the sgRNA is not detected at either $\mathrm{p} 1$ or $\mathrm{p} 10$, we set $\mathrm{nr}_{p 1}=0$ or

$1323 \mathrm{nr}_{p 10}=0$, correspondingly.

1324 With that, we calculated $\log 2 \mathrm{fc}$ of all $\operatorname{sgRNAs}$ in the dish.

1325 Step 3. At first, we made some deductions. In the dish, we assumed the number of the negatively-selected sgRNAs is no more than $20 \%$ of the non-selected sgRNAs. We

1327 assumed the $\log 2 \mathrm{fc}$ of the former ones follows a normal distribution $\mathrm{N}\left(\mathrm{u}, \sigma^{2}\right)$, and

1328 the $\log 2 \mathrm{fc}$ of the latter ones follows a normal distribution $\mathrm{N}\left(0, \sigma^{2}\right)$. Here $\mathrm{u}>0$ and

$1329 \mathrm{u}>4 \sigma^{2}$. Then the $\log 2 \mathrm{fc}$ of sgRNAs in the dish follows a mixture-normal

1330 distribution. The mode, which is the value occurring a maximum number of times in

1331 the distribution, will indicate the center of the normal distribution of the log $2 \mathrm{fc}$ from

1332 the non-selected sgRNAs and should be zero.

1333 Then, we conducted the analysis. We calculated the distribution of $\log 2 \mathrm{fc}$ of all 1334 sgRNAs in the dish, find mode $(m)$ of the distribution and correct $\log 2 \mathrm{fc}$ of the 1335 sgRNAs with the following formula:

$$
\log 2 \mathrm{fc} \_\mathrm{m}=\log 2 \mathrm{fc}-\mathrm{m} .
$$


1337 With that, the log2fc_m of sgRNAs in the dish follows a mixture-normal distribution.

1338 The mode of the distribution indicates the center of the normal distribution of the log

1339 fold change from the non-selected sgRNAs and is zero.

1340 Step 4. Again, we made some deductions at first. The previous deductions in step 3

1341 demonstrated the $\log 2 \mathrm{fc} \_\mathrm{m}$ of the negatively-selected sgRNAs follows the normal

1342 distribution $N\left(u, \sigma^{2}\right)$. We have assumed that $u>4 \sigma^{2}$, thus the negatively-selected

1343 sgRNAs whose log2fc_m $<0$ should occupy $4.5 \%$ of all negatively-selected sgRNAs.

1344 However, the percent of negatively-selected sgRNAs is less than $20 \%$ of non-selected

1345 sgRNAs in the dish. Thus the negatively selected sgRNAs whose $\log 2 \mathrm{fc} \_\mathrm{m}<0$

1346 should occupy less than $1 \%(0.2 /(1+0.2) \times 0.045=0.0075) \operatorname{sgRNAs}$ in the dish. Since this

1347 percent is relatively small, we assumed that the sgRNAs with $\log 2 \mathrm{fc} \_\mathrm{m}<0$ are from

1348 the non-selected sgRNAs but not from the negatively-selected sgRNAs.

1349 Then, we conducted the analysis. We randomly picked a sgRNA $k$ with the positive

$1350 \log 2 \mathrm{fc} \_\mathrm{m}\left(\log 2 \mathrm{fc}_{-} \mathrm{m}_{k}\right)$ from the dish. We took the sgRNAs in the dish whose

$1351 \log 2 \mathrm{fc}_{-} \mathrm{m}<-\log 2 \mathrm{fc}_{-} \mathrm{m}_{k}$ being from the non-selected sgRNAs, and recorded their

1352 number as $x_{1}$. We counted the ones whose $\log 2 \mathrm{fc} \_\mathrm{m}>\log 2 \mathrm{fc}_{-} \mathrm{m}_{k}$ in the dish as $x_{2}$.

1353 If $x_{1}>0$ and $x_{2}>x_{1}$, then we can deduce that $x_{1}$ sgRNAs are from the non-

1354 selected sgRNAs and $x_{2}-x_{1}$ sgRNAs are from the negatively-selected ones in all the

$1355 x_{2}$ sgRNAs. Then, taking $\log 2 \mathrm{fc}_{-} \mathrm{m}_{k}$ as the threshold, we can calculate the false

1356 discovery rate $\left(\mathrm{fdr}_{k}\right)$ of the negatively-selected sgRNAs as:

$$
\mathrm{fdr}_{k}=x_{1} / x_{2}
$$


1358

1359

1360

1361

1362

1363

1364

1365 identified the sgRNAs whose $\log 2 \mathrm{fc}_{-} \mathrm{m}>\log 2 \mathrm{fc}_{-} \mathrm{m}_{l}$ in the dish as the ssgRNA.

1366 However, if we cannot find any pair whose $\mathrm{fdr}<0.1$ in pairs set, we claimed no

1367 ssgRNA in the dish.

1368 Step 6. We can get the ssgRNAs by conducting the steps 1-5. However, some ssgRNAs

1369 may not be saturated sequenced and have small reads counts at either $\mathrm{p} 1$ or $\mathrm{p} 10$. The

1370 change of their abundance from $\mathrm{p} 1$ to $\mathrm{p} 10$ was suspected. With that, we modified step

13712 to give weights to the $\log 2$ fold change $(\log 2 \mathrm{fc})$ of the $\operatorname{sgRNAs}$ in the dish. At first,

1372 we selected the larger one of its normalized reads counts (nr) at p1 and p10 for each

1373 sgRNA. Then we ranked all sgRNAs by their selected $\mathrm{nr}$ in descending order. Next,

1374 we calculated its percentile (perct) for each sgRNA in the rank list. At last, picking a

$1375 \operatorname{sgRNA} z$ with the percentile value $\operatorname{perct}_{z}$, we calculated its $\log 2 \mathrm{fc}\left(\log 2 \mathrm{fc}_{z}\right)$ with

1376 the following formula,

$$
\log 2 \mathrm{fc}_{z}=\operatorname{perct}_{z} * \log 2\left(\frac{\max \left\{\mathrm{nr}_{p 1}, 10\right\}}{\max \left\{\mathrm{nr}_{\left.p_{10}, 10\right\}}\right.}\right)
$$


where $\mathrm{nr}_{p 1}$ and $\mathrm{nr}_{p 10}$ are the normalized reads count of the sgRNA at $\mathrm{p} 1$ and $\mathrm{p} 10$.

1379 However, if the sgRNA is not detected at either $\mathrm{p} 1$ or $\mathrm{p} 10$, we set $\mathrm{nr}_{p 1}=0$ or $1380 \mathrm{nr}_{p 10}=0$, correspondingly.

1381 We modified the $\log 2 \mathrm{fc}$ of all sgRNAs in the dish by this approach.

1382 Finally, we conducted step 1,6,3,4 and 5 to detect ssgRNAs in the dish.

\section{Evaluation of Negative-SgRNA-Shot method}

1385 We designed a simulation experiment to test the performance of NSgRNAShot.

1386 At first, we generated a series of simulated datasets.

1387 Step 1. We picked a dish. We conducted steps 1 to 3 of NSgRNAShot to get the

$1388 \log 2 \mathrm{fc} \_\mathrm{m}$ of all the sgRNAs in the dish. We also recorded the normalized reads count

1389 of the $\operatorname{sgRNAs}$ at $\mathrm{p} 10\left(\mathrm{nr}_{p 10}\right)$. For the sgRNAs who got no reads count at $\mathrm{p} 10$, we set 1390 their $\mathrm{nr}_{p 10}$ as zero.

1391 Step 2. We counted sgRNAs whose $\log 2 \mathrm{fc} \_\mathrm{m}<0$ in the dish and recorded the 1392 number as $r$. We randomly picked a sgRNA $p$ from the $r$ sgRNAs and recorded its $1393 \log 2 \mathrm{fc}_{-} \mathrm{m}$ as $\log 2 \mathrm{fc}_{-} \mathrm{m}_{p}$. Then we tried to find a $\operatorname{sgRNA} q$ and recorded its $\log 2 \mathrm{fc} \_\mathrm{m}$ 1394 as $\log 2 \mathrm{fc} \_\mathrm{m}_{q}$ where $\left|\log 2 \mathrm{fc}_{-} \mathrm{m}_{p}+\log 2 \mathrm{fc}_{-} \mathrm{m}_{q}\right|<0.05$, thus we got a sgRNAs pair 1395 whose log2fc_m values are symmetrical about zero.

1396 We repeated the process $r / 2$ times, got $r / 2$ sgRNAs pairs to form a sgRNAs set 1397 whose log2fc_m values are symmetrical about zero. Since we had recorded the $\mathrm{nr}_{p 10}$ 
Pan et al 2020

1398

1399

1400

1401

1402

1403

1404

1405

1406 We had recorded the $\mathrm{nr}_{p 10}$ of these sgRNAs, we inferred their normalized reads count

1407 of the $\operatorname{sgRNAs}$ at $\mathrm{p} 1\left(\mathrm{nr}_{\mathrm{p} 1}\right)$ with the following formula,

1408

1409 Thus we got a sgRNAs set with the normalized reads count at both $\mathrm{p} 1$ and $\mathrm{p} 10$. We saw

1410 the set as the simulated negatively-selected sgRNAs set. Finally, we combined the

1411 simulated non-selected and negatively-selected sgRNAs sets to get a simulated sgRNAs

1412 dataset.

1413 Step 4 . We took $(\alpha, \beta)$ in step 3 as the parameter pair. We set the parameter pairs as

$1414(0.01,6),(0.01,8),(0.05,4),(0.05,6),(0.05,8),(0.1,4),(0.1,6),(0.1,8),(0.2,4),(0.2,6)$,

1415 and $(0.2,8)$. We repeated steps 3 and 4 one hundred times with each parameter pair to

1416 generate the simulated datasets. We randomly took a dataset under each parameter pair.

1417 We plotted the normalized reads count of the non-selected and negatively selected

1418 sgRNAs in the dataset and calculated the distribution of the $\log 2$ fold change of these 
1419

1420

1421

1422

1423

1424

1426

sgRNAs. The scatter plot and distribution plot showed that the simulation is successful because they tend to fit the two plots in the real dataset (Figure S1a).

To this step, we had generated a series of simulated datasets. Then, we tested the performance of NSgRNAShot on detecting the negatively-selected sgRNAs at the simulated datasets. We used two indicators-precision (prec) and recall (rec), which are calculated with the following formula, to benchmark NSgRNAShot,

$$
\text { prec }=\frac{T P}{T P+F P}, \text { and } r e c=\frac{T P}{T P+F N},
$$

where $T P$ is the number of negatively-selected sgRNA identified as ssgRNA; $F P$ is the number of non-selected sgRNA identified as ssgRNA; $F N$ is the number of negatively-selected sgRNA failed to be identified as ssgRNA.

We run NSgRNAShot on the simulated datasets to detect negatively-selected sgRNAs.

We can see that in the datasets whose parameter pairs are $(0.05,8),(0.1,6),(0.1,8)$, $(0.2,6)$, and $(0.2,8)$, the precision and recall indicators are both higher than $80 \%$ (Table S1). We believed NSgRNAShot could successfully identify the negatively-selected sgRNAs in the circumstances.

\section{References}

1. Shalem, O. et al. Genome-scale CRISPR-Cas9 knockout screening in human cells. Science 343, 84-87 (2014).

2. Chen, S. et al. Genome-wide CRISPR screen in a mouse model of tumor growth and metastasis. Cell 160, 1246-1260 (2015).

3. Kim, J., Cantor, A.B., Orkin, S.H. \& Wang, J. Use of in vivo biotinylation to study protein-protein and protein-DNA interactions in mouse embryonic stem cells. Nature protocols 4, 506-517 (2009). 
1446 4. Tzelepis, K. et al. A CRISPR Dropout Screen Identifies Genetic Vulnerabilities and 1447 Therapeutic Targets in Acute Myeloid Leukemia. Cell Rep 17, 1193-1205 (2016).

14485 . Li, W. et al. MAGeCK enables robust identification of essential genes from 1449 genome-scale CRISPR/Cas9 knockout screens. Genome biology 15, 554 (2014).

1450 6. Bodapati, S., Daley, T.P., Lin, X., Zou, J. \& Qi, L.S. A benchmark of algorithms for the 1451 analysis of pooled CRISPR screens. Genome biology 21, 62 (2020).

1452 7. Shohat, S. \& Shifman, S. Genes essential for embryonic stem cells are associated 1453 with neurodevelopmental disorders. Genome research 29, 1910-1918 (2019). 
Figures

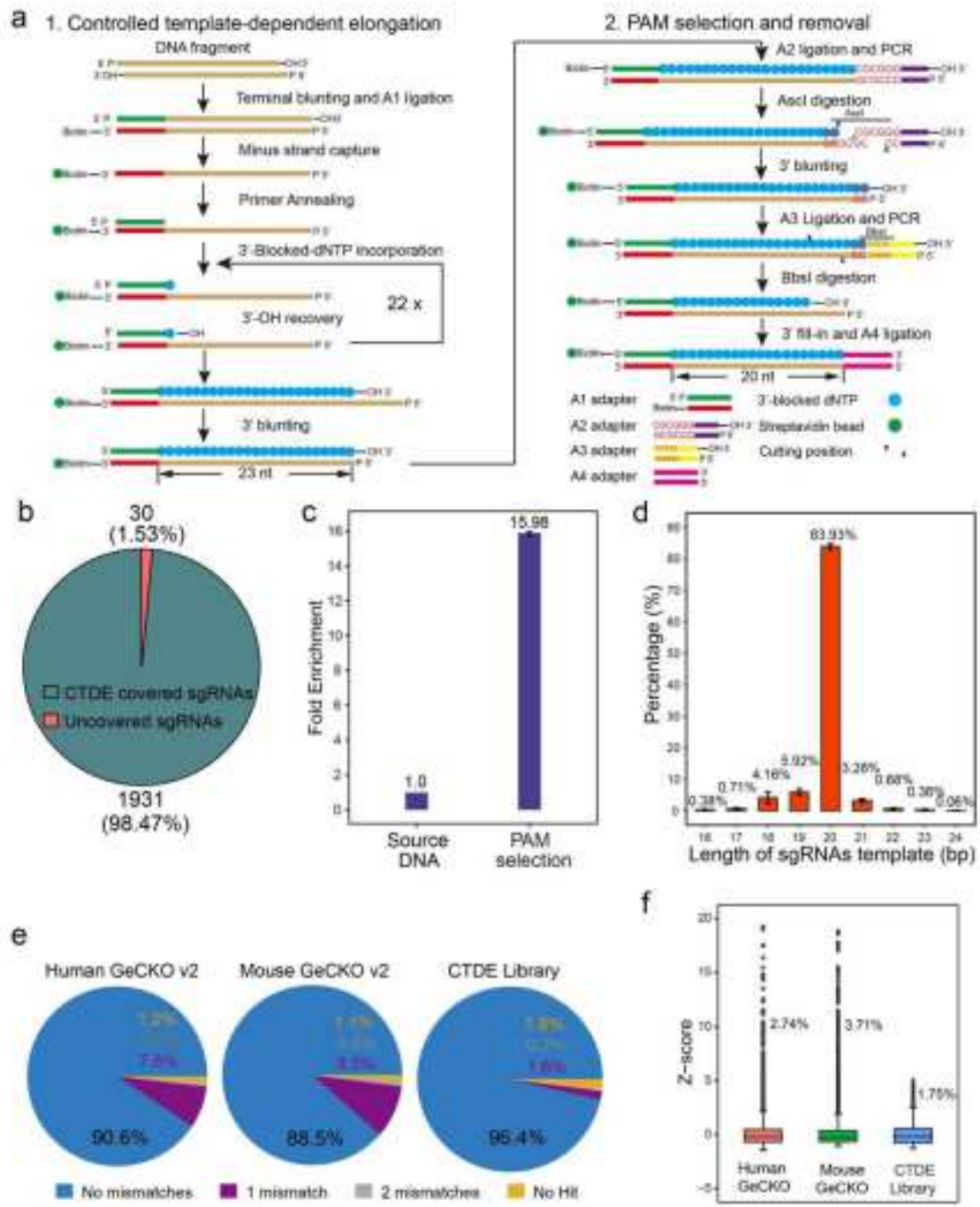

\section{Figure 1}

[Please see the manuscript file to view the figure caption.] 

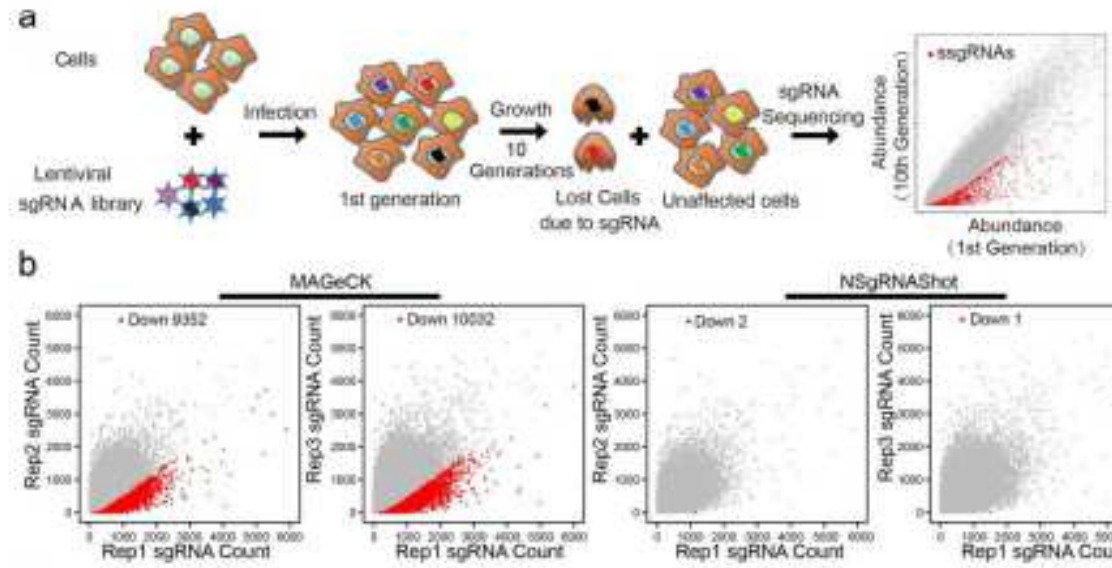

NSGRNASho:
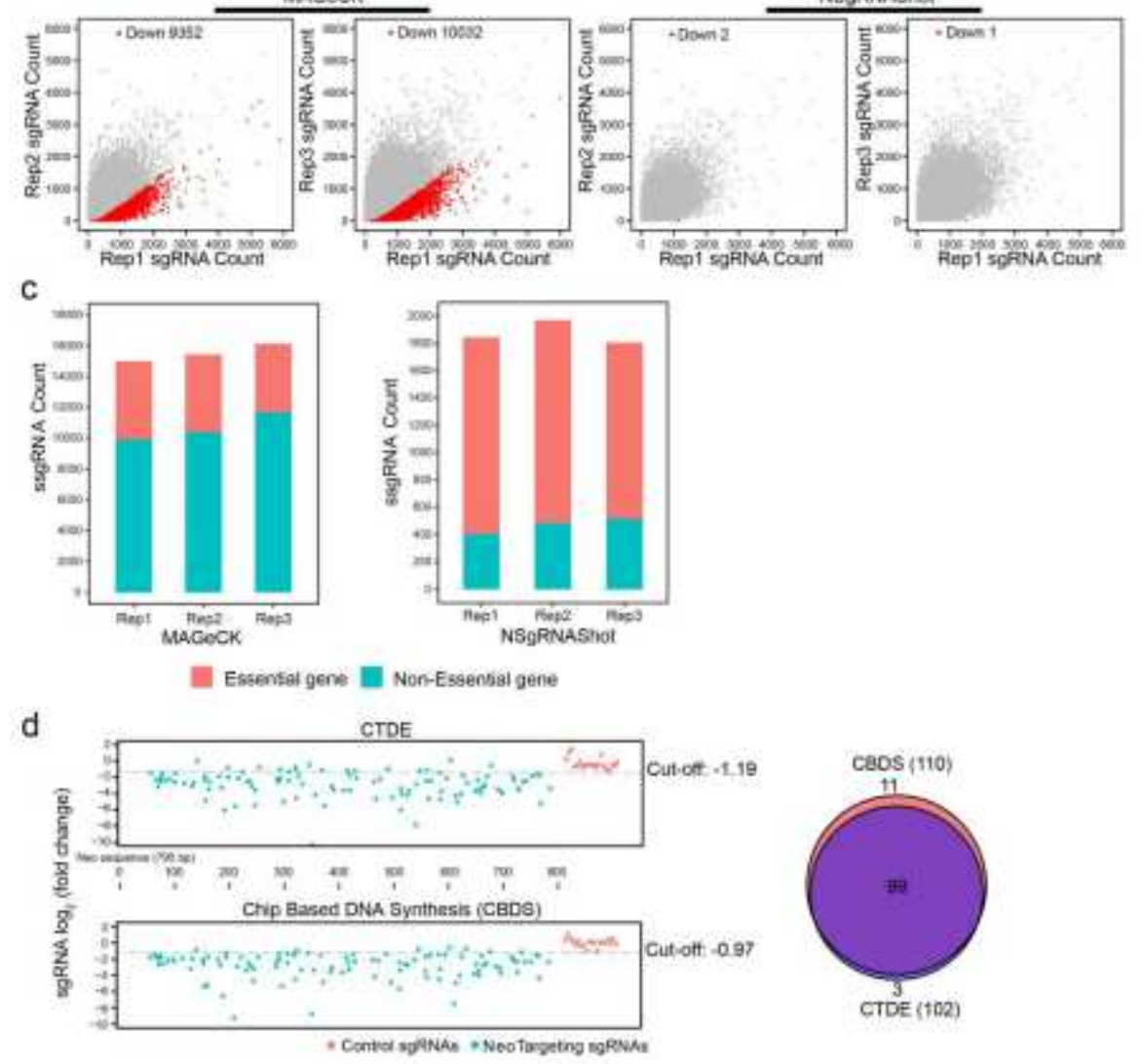

Figure 2

[Please see the manuscript file to view the figure caption.] 
a
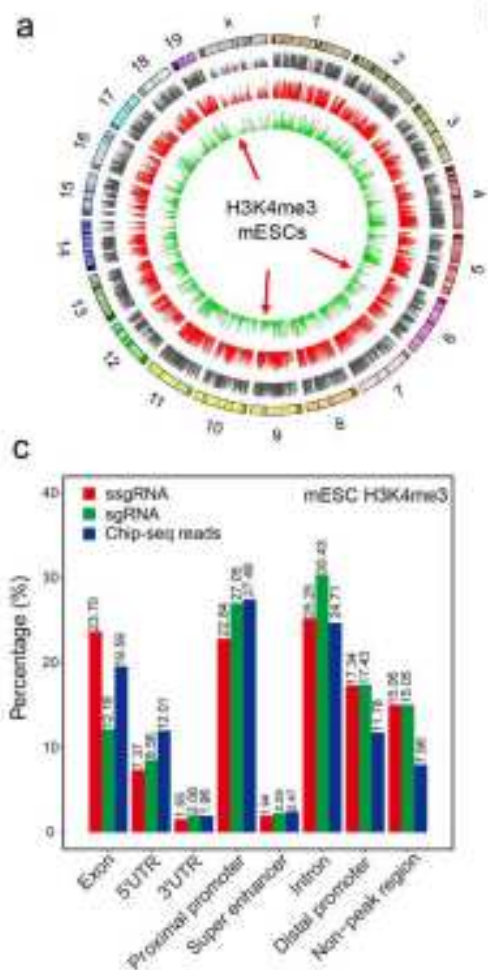

Figure 3

b

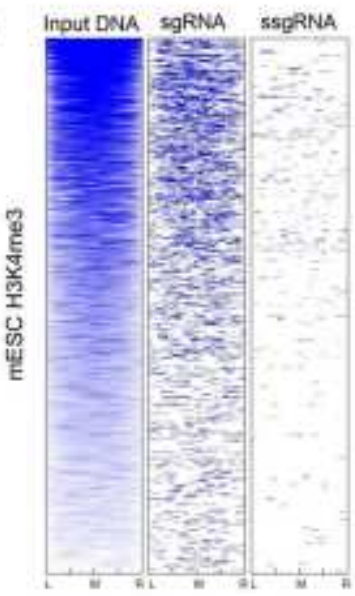

f

CDS targeted by sgRNA COS targeted by ssgRNA

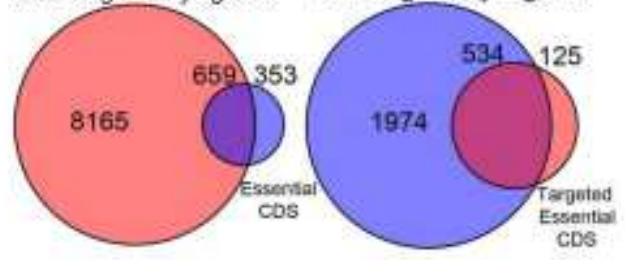

d

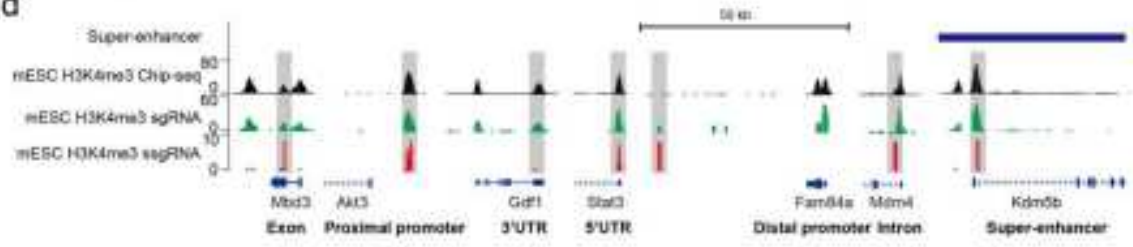

e

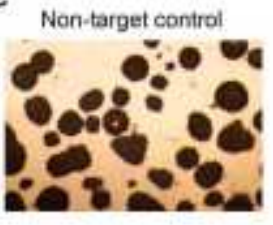

che 7.35871655

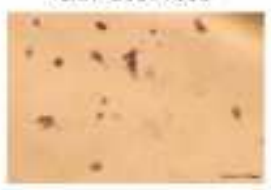

chr19-61275641

chr12-86827179
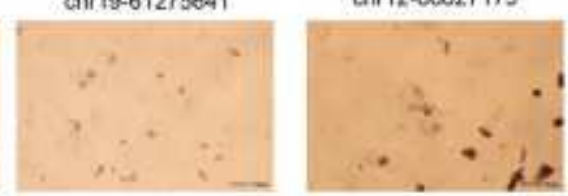

Figure 3

[Please see the manuscript file to view the figure caption.] 
Figure 4

a

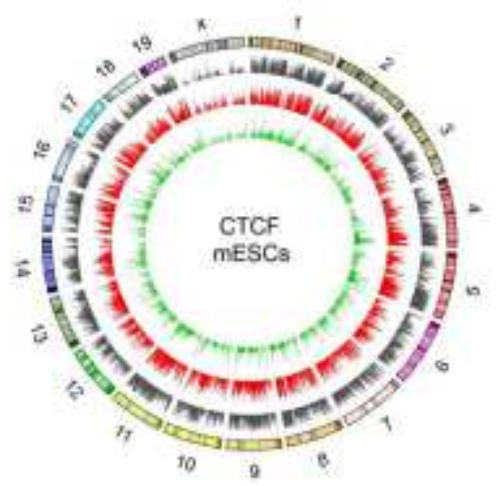

b
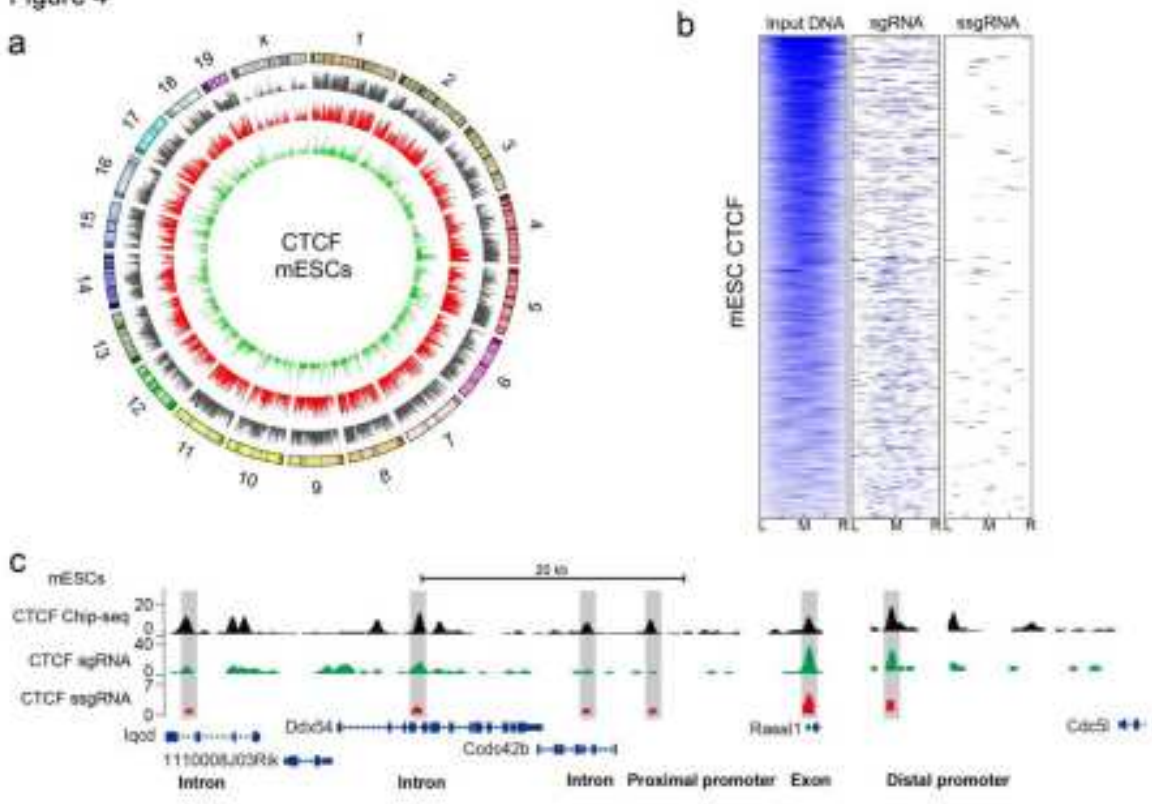

d

Non-tame-comol

chr19-21447441

Chr15-12027231

ar14-6307a202


Essental common peaks $1857(71.15 \%)$



cethyse upecific peak: $765026.05 \%$

\section{Figure 4}

[Please see the manuscript file to view the figure caption.] 
Figure 5

a

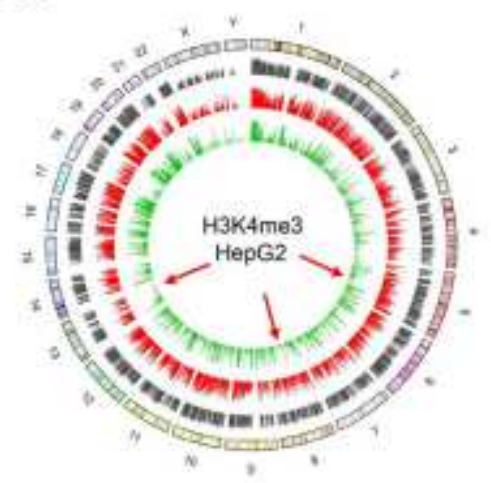

b InOUt DNA SgRNA SsgRNA

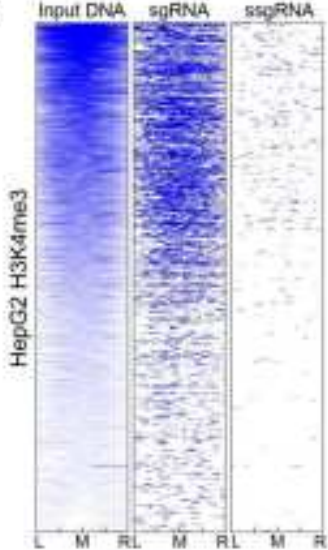

C

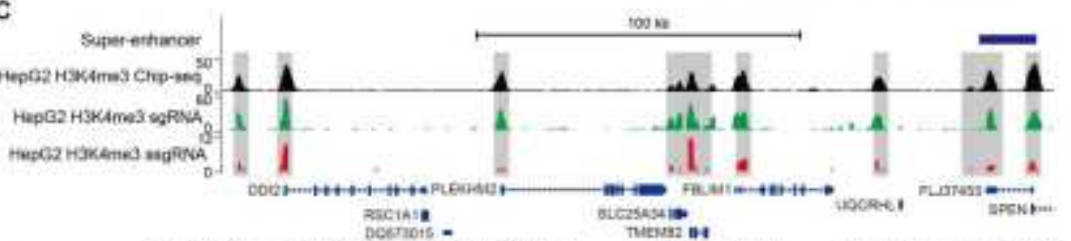

d

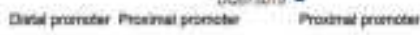
Tuevan an

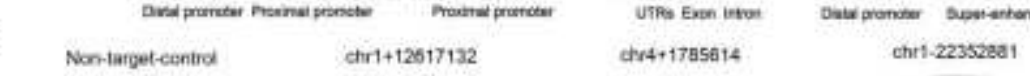

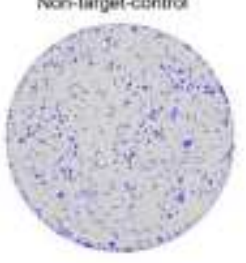

e

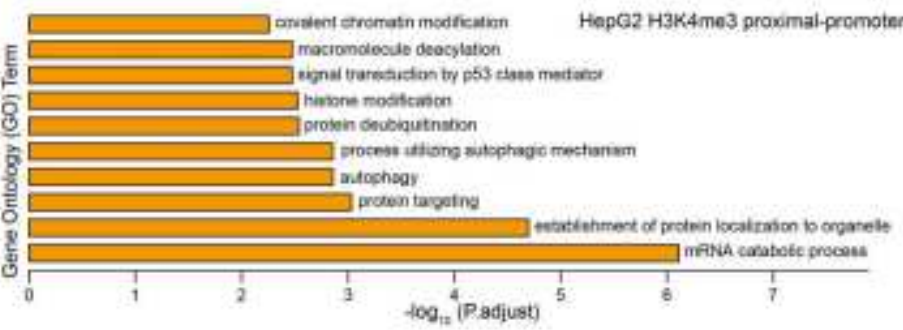

Figure 5

[Please see the manuscript file to view the figure caption.] 
Figure 6

a

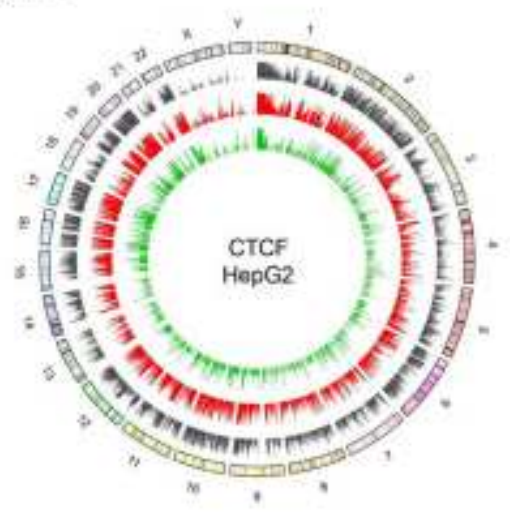

b

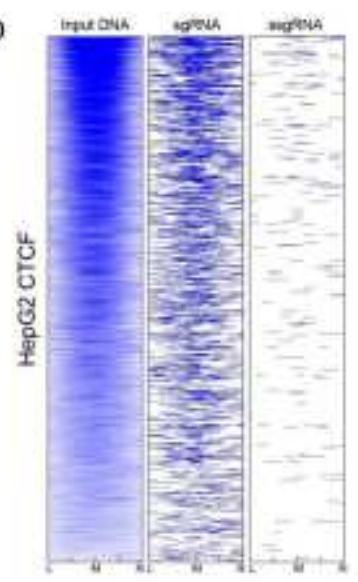

c

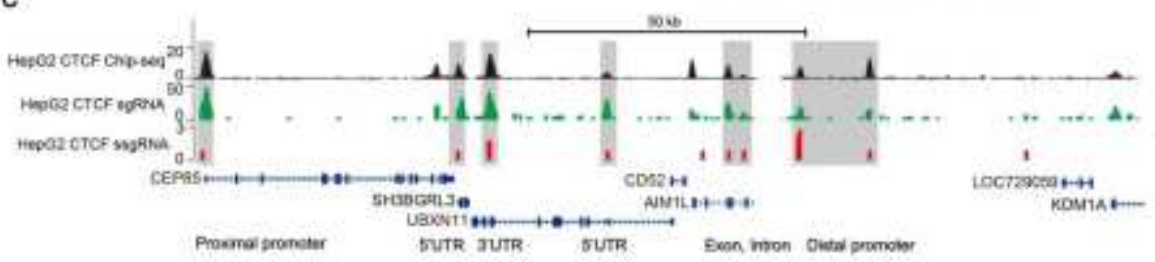

d

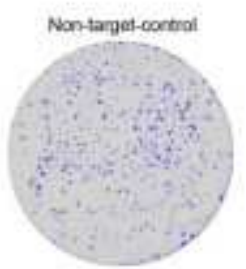

e HepG2 CTCF introns
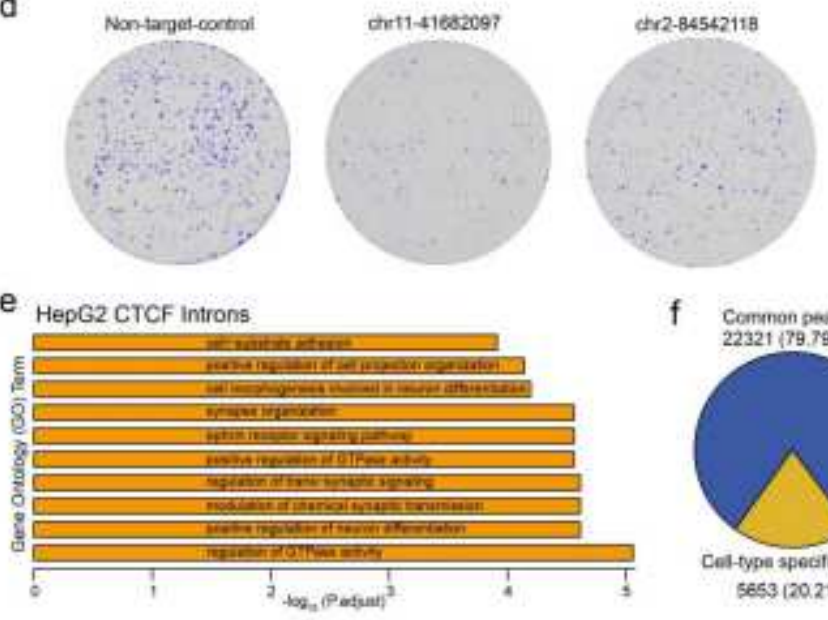

ctrr2+114361824

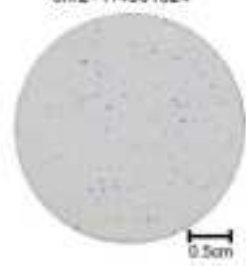

f Common peste

Essential conmon peaks


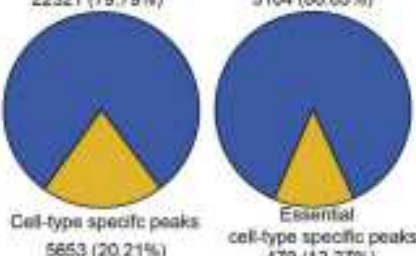

\section{Figure 6}

[Please see the manuscript file to view the figure caption.] 
Figure 7
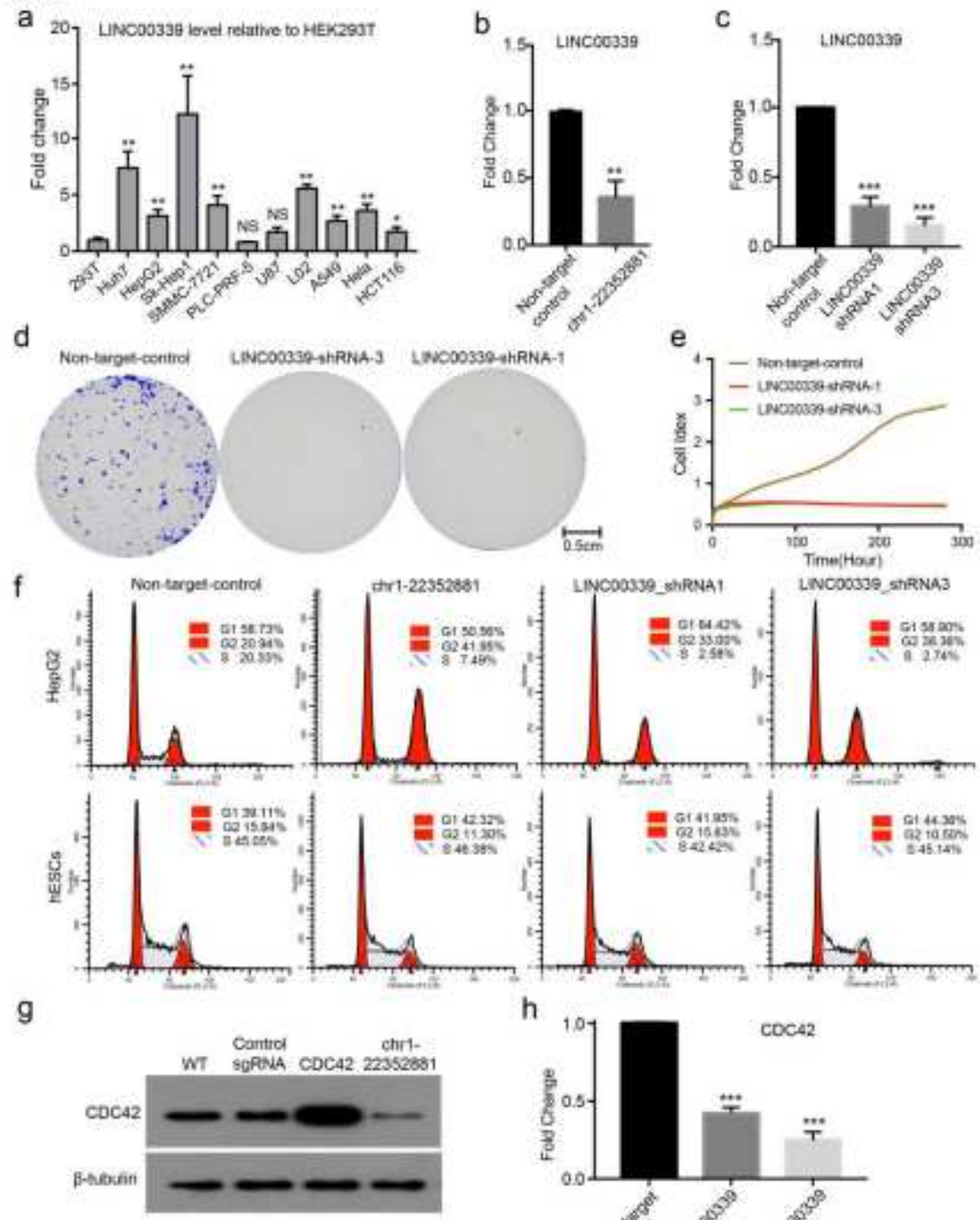

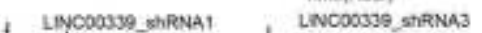
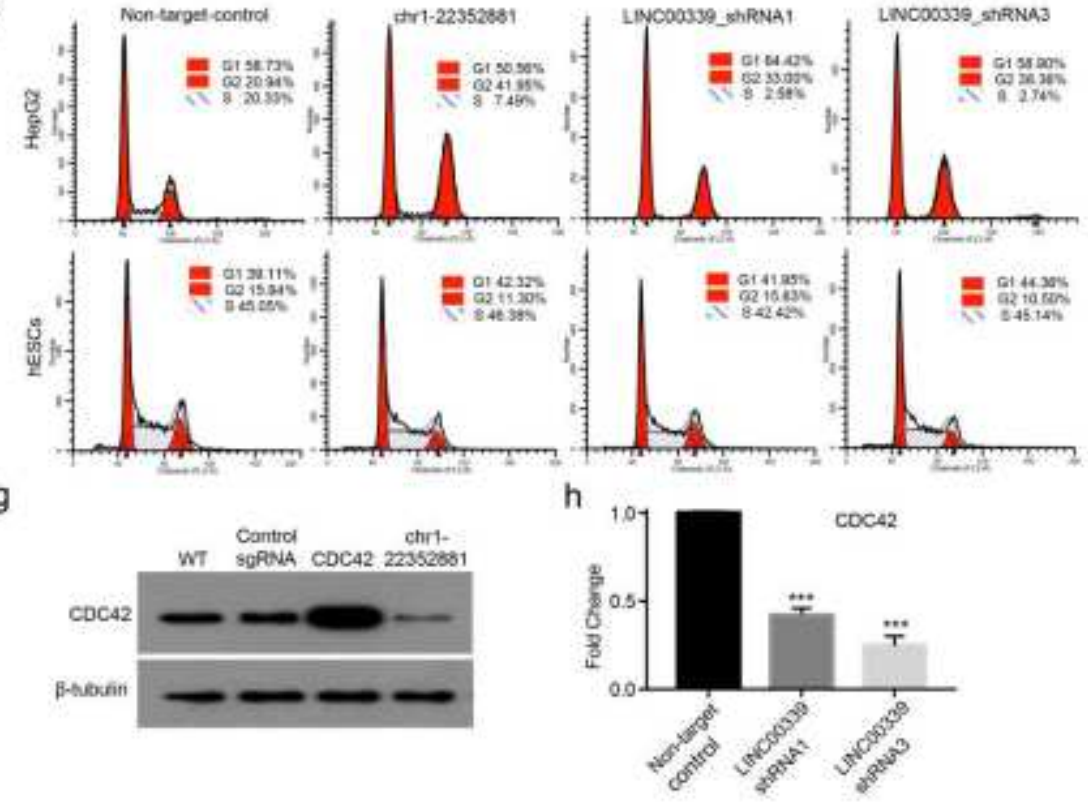

Figure 7

[Please see the manuscript file to view the figure caption.]

\section{Supplementary Files}

This is a list of supplementary files associated with this preprint. Click to download.

- SupplementalTable.zip 